Estruturas de vizinhanças espaciais nos modelos autorregressivos e de médias móveis espaço-temporais STARMA

\author{
Esther Yanfei Jin
}

DissertaÇÃo APRESENTADA

AO

Instituto de Matemática e Estatística

DA

Universidade de São Paulo

PARA

OBTENÇÃ̃ DO TÍTULO

DE

Mestre em Ciências

Programa: Programa de Pós-graduação em Estatística

Orientador: Profa. Dra. Chang Chiann

São Paulo, 18 de janeiro de 2017 


\section{Estruturas de vizinhanças espaciais nos modelos autorregressivos e de médias móveis espaço-temporais STARMA}

Esta versão da dissertação contém as correções e alterações sugeridas pela Comissão Julgadora durante a defesa da versão original do trabalho, realizada em 25/05/2017. Uma cópia da versão original está disponível no

Instituto de Matemática e Estatística da Universidade de São Paulo.

Comissão Julgadora:

- Profa. Dra. Chang Chiann (orientadora) - IME-USP

- Profa. Dra. Clélia Maria de Castro Toloi - IME-USP

- Profa. Dra. Thelma Sáfadi - UFLA 
Dedico este trabalho a Deus, aos meus pais, parentes e amigos por todo apoio e contribuição que me prestaram 


\section{Agradecimentos}

Durante o mestrado, tive tantas ajudas e apoios que gostaria de agradecer. E agora finalmente chegou este momento, mas estou com dificuldade para escrever, talvez seja difícil encontrar palavras que consigam expressar o que estou sentindo.

Primeiro de tudo, gostaria muito de agradecer a Deus por sempre me guiar e fortalecer para seguir em frente com os meus sonhos e não desanimar com as dificuldades e tribulações. Também agradeço a Ele por ter colocado na minha vida tantas pessoas que nunca me deixaram só; sem elas, não seria possível a realização deste trabalho.

Agradeço aos meus pais, quem me deram a vida, cuidaram de mim, ficaram ao meu lado e me apoiaram nos momentos difíceis. Principalmente ao meu pai, quem sempre me motivou e me mostrou a importância dos estudos. Me encorajou a não desistir de estudar por causa da imensa dificuldade da língua portuguesa nos meus primeiros anos no Brasil.

Agradeço à minha orientadora Chang Chiann pelo conhecimento, carinho e paciência. Para mim, ela não é só minha professora, mas também uma amiga. Nas horas em que parecia que nada do trabalho estava dando certo, ela sempre foi a conselheira que me deu consolo e encorajamento. Agradeço às professoras da banca pelas importantes observações e sugestões. Aos demais professores do IME pelos esses anos de ensinamentos.

Agradeço aos meus amigos Yunyao Yan, Louise Rossi, Helder Alves, Victor Gonçalves e João Paulo, quem me acompanharam todos esses anos de aprendizado. Obrigada pela amizade constante, encorajamento e ajuda. Com certeza, nunca vou esquecer os momentos que passamos juntos.

Agradeço às minhas amigas Verônica Paukstys, Giulia Akemi, Karoline Marquezi, Weiya Lin e Yali Lu, quem sempre me encorajaram, apoiaram e me deram consolos nas horas que precisava.

Agradeço a todos os funcionários da USP, principalmente do IME, por tudo que vocês contribuíram para este ambiente agradável de estudo. IME-USP ficará sempre na minha memória.

Por último, agradeço a todos aqueles que esqueci de mencionar, por terem me ajudado nesta importante fase da minha vida, que é somente o começo... 


\section{Resumo}

O objetivo deste trabalho é comparar as estruturas de vizinhanças espaciais ou matrizes de pesos espaciais da classe de modelos autorregressivos e de médias móveis espaçotemporais (STARMA). O modelo STARMA é empregado para descrever dados de séries temporais espacialmente localizados, ele é caracterizado pela dependência linear defasada tanto no espaço quanto no tempo. Foram realizadas simulações utilizando vários modelos de covariância espaço-temporal para comparar diferentes estruturas de construção da matriz de pesos espaciais com a finalidade de identificar a melhor matriz. As matrizes espaciais com pesos exponenciais apresentaram os melhores desempenhos de ajuste dos modelos STAR; e mostram uma estabilidade em relação à medida de ajuste. Por fim para ilustração, será ajustado um modelo STARMA para um conjunto de dados mensais do índice FIPEZAP de preço imobiliário de venda para apartamentos de dois dormitórios de seis cidades metropolitanas de São Paulo.

Palavras-chave: séries temporais, espaço-temporais, STARMA, vizinhanças, matrizes, pesos espaciais, exponenciais. 


\section{Abstract}

The objective of this work is to compare spatial neighborhoods structures, or the same as spatial weights matrices of the class of space-time autoregressive and moving average models STARMA. The STARMA model is used to describe spatially localized time series datas, it is characterized by the linear dependence lagged both in space and time. Simulations were performed using several space-time covariance models to compare different structures of construction of the weight matrix with the purpose of identifying the best matrix. The spatial matrices with exponential weights presented the best adjustment performances of the STAR models ans showed a stability in relation to the adjustment measure. Finally, for illustration, a STARMA model will be adjusted for a set of monthly data of the FIPEZAP real estate price index for two bedroom apartments in six metropolitan cities of São Paulo.

Keywords: time series, space-time, STARMA, neighborhoods, matrices, spatial weights, exponential. 


\section{Sumário}

\begin{tabular}{lll}
\hline 1 & Introdução & 1
\end{tabular}

2 Conceitos Básicos 4

2.1 Processo Estocástico . . . . . . . . . . . . . . . . . . . . . . . . . . . . . 5

2.2 Processo Estacionário . . . . . . . . . . . . . . . . . . . . . . . . . . . . . 6

2.3 Modelos Box e Jenkins . . . . . . . . . . . . . . . . . . . . . . . . . . . . . . 8

2.3 .1 Modelo Autorregressivo AR $(\mathrm{p})$. . . . . . . . . . . . . . . . . 9

$2.3 .2 \quad$ Modelo Média Móvel MA (q) . . . . . . . . . . . . . . . . . . . . . 9

$2.3 .3 \quad$ Modelo Autorregressivo e Média Móvel ARMA (p,q) . . . . . . . . 9

2.3 .4 Modelo ARIMA $(\mathrm{p}, \mathrm{d}, \mathrm{q})$. . . . . . . . . . . . . . . 10

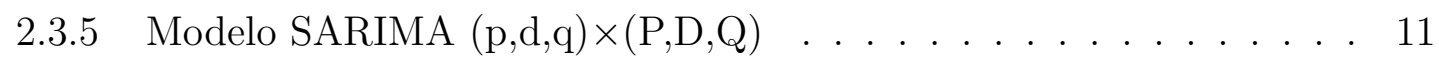

2.4 Processos Iterativos $\ldots \ldots \ldots \ldots \ldots$

2.4 .1 Identificação . . . . . . . . . . . . . . . . . . . . 12

2.4 .2 Estimação . . . . . . . . . . . . . . . . . . . . . . 15

2.4 .3 Diagnóstico . . . . . . . . . . . . . . . . . . . 16

2.4 .4 Previsão . . . . . . . . . . . . . . . . . . . . . . 17

$\begin{array}{llr}3 & \text { Modelos STARMA } & 19\end{array}$

$3.1 \quad$ Matriz de Peso Espacial $\mathrm{W}^{(l)}$. . . . . . . . . . . . . . . . . . . 20

3.2 Modelos STARMA $\ldots \ldots \ldots \ldots \ldots \ldots$. . . . . . . . . . . . . . . . 23

3.3 Identificação . . . . . . . . . . . . . . . . . . . . . 25

3.4 Estimação $\ldots \ldots \ldots \ldots$. . . . . . . . . . . . . . . . . . . . . . . . . . . . . . 28

3.4 .1 Método de Máxima Verossimilhança . . . . . . . . . . . . . . . 28

3.4 .2 Estimação por Filtro de Kalman . . . . . . . . . . . . . . . . . . . . 31

3.5 Diagnóstico . . . . . . . . . . . . . . . . . . . 36

3.6 Previsão . . . . . . . . . . . . . . . . . . . . . . . . . 36

\begin{tabular}{lll}
\hline & Estudo de Simulação & 38
\end{tabular}

4.1 Modelos de Covariância Espaço-Temporal . . . . . . . . . . . . . . . . . . 38

4.2 Descrição dos Processos de Simulação . . . . . . . . . . . . . . . . . . . . . 41

4.3 Avaliação de Ajuste . . . . . . . . . . . . . . . . . . . . . . . . . . . . . . . 43 
4.4 Resultado de Simulação . . . . . . . . . . . . . . . . . . . . . . . . . . 44

4.5 Conclusão de Simulação $\ldots$. . . . . . . . . . . . . . . . . . . . . . 44

5 Aplicação

5.1 Índice de Preço Imobiliário de Venda . . . . . . . . . . . . . . . . . . . . . 53

$5.1 .1 \quad$ Descrição de Dados . . . . . . . . . . . . . . . . . . . . . . . . . . 53

5.1 .2 Análise Descritiva . . . . . . . . . . . . . . . . . . 55

5.1 .3 Identificação, Estimação, Diagnóstco e Previsão . . . . . . . . . . . 56

\begin{tabular}{lll}
\hline 6 & Conclusões e Estudos Futuros & 65
\end{tabular}

\begin{tabular}{ll}
\hline Referências Bibliográficas & 67
\end{tabular} 


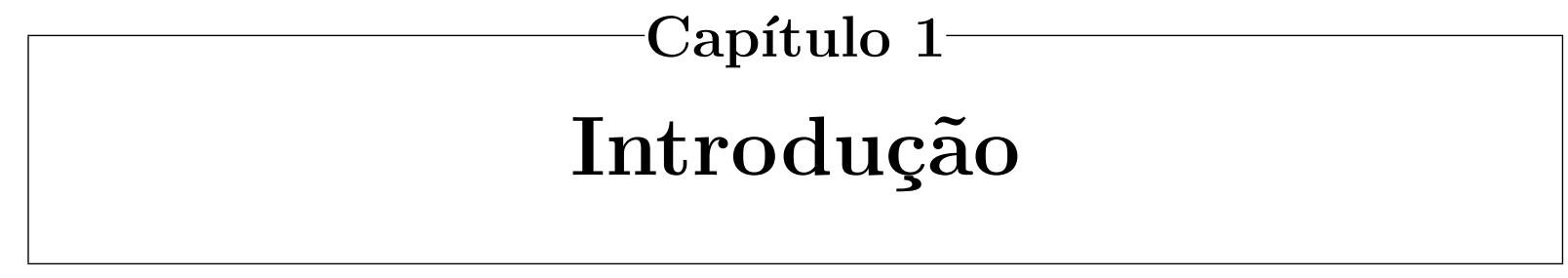

Geoestatística, uma classe da estatística espacial que incorpora a dependência espacial aos modelos estatísticos para variáveis geo-referenciadas, intimamente associado com a distribuição estatística dos dados no espaço. E sob determinadas hipóteses, torna-se possível fazer inferências e predições a partir de amostras observadas. Vários métodos e técnicas foram desenvolvidos ao longo dos últimos cinquenta anos, dentre os quais se destaca, por exemplo, o krigagem (Sassi (2016)), que calcula um valor de um local utilizando os dados ao redor espacialmente localizados através de uma função de correlação espacial entre eles. Um dos modelos de regressão espacial mais conhecido é o denominado SAR (Spatial Autoregressive Model). Este é um modelo espacial nascido na área de econometria espacial que incorpora a dependência espacial de modo global, o que pressupõe uma homogeneidade e estacionariedade espacial. Mais detalhes se encontram em Anselin (2013). Outro modelo bastante conhecido é CAR (Conditional Autoregressive Model), na abordagem de modelos CAR, a distribuição da observação de uma localização é especificamente condicional às regiões vizinhas (Anselin (2013)).

No contexto geoestatístico de espaço-temporal, os modelos clássicos são modelos STARMA (Autorregressivos e de Médias Móveis Espaço-temporais). O modelo STARMA foi desenvolvido principalmente nas décadas de 80 por Pfeifer e Deutsch (Pfeifer e Deutsch (1980a)), depois passou por um tempo de esquecimento por causa da dificuldade de implementação computacional. Até os últimos anos, ele foi retomado e é frequentemente usado em análises geoestatísticas para descrever séries temporais espacialmente localizadas, caracterizado pela dependência linear defasada tanto no espaço quanto no tempo.

Pace et al. (2000) ajustaram modelo STAR (Autorregressivos Espaço-temporais) para dados de preços de imóveis da cidade Baton Rouge do estado Luisiana dos EUA. Comparando com modelos tradicionais univariados que somente consideram a estrutura temporal, o modelo STAR mostrou o melhor desempenho em ajuste por levar em conta a característica espacial nos dados.

Giacomini e Granger (2004) simularam várias séries para 4, 6, 9 e 16 regiões usando modelos STAR para efeito de comparação entre os modelos STAR, VAR e ARMA univariado. O resultado mostrou que o modelo STAR ajusta melhor as séries de espaço-temporal. 
Rao e Antunes (2004) usaram a classe de modelos STARMA e SARIMA para ajustar as séries de temperaturas mensais de 9 regiões do Reino Unido, os resultados de ajuste mostraram a eficiência do modelo STARMA no sentido de obter as melhores previsões.

Martins (2013) aplicou a classe de modelos STARMA e SARIMA nos dados de temperaturas mínimas mensais de 8 estações meteorológicas situadas no Paraná, constatou-se que ambos os modelos se ajustaram de maneira satisfatória aos dados, produzindo previsões acuradas.

Kamarianakis e Prastacos (2005) aplicou a classe de modelos STARMA nos dados de fluxo de tráfego (o número total de veículos que passam em um determinado ponto durante um dado intervalo de tempo) da cidade Atenas, Grécia. O estudo mostrou desejável desempenho de previsões. Concluiu que o modelo STARMA pode ser uma ferramenta útil nos casos em que o pesquisador enfrenta conjuntos de dados espaço-temporais.

Glasbey e Allcroft (2008) aplicaram modelo STARMA nos dados de radiação solar registrada em dez locais com o intervalo de dez minutos nos montes de Pentland do sul de Edimburgo, a capital da Escócia no Reino Unido. O modelo STAR foi escolhido como o melhor modelo para descrever a dependência espacial da radiação.

Zhou e Buongiorno (2006) desenvolveram um modelo econométrico espaço-temporal usando modelo STARMA para os preços trimestrais de madeira de serraria de pinho de 21 regiões geograficamente contíguas no sul dos Estados Unidos cobrindo 11 estados (Alabama, Arkansas, Flórida, Geórgia, Louisiana, Mississippi, Carolina do Norte, Carolina do Sul, Tennessee, Texas e Virgínia). As correlações entre os preços nas regiões vizinhas ajudaram a prever preços futuros.

Shoesmith 2013) utilizou modelo STARMA para prever as taxas de crime de violência (roubo, assalto, assassinato, etc) em 9 regiões do estado de Flórida. O modelo STAR foi detectado como o melhor modelo para descrever a dependência criminal das regiões em estudo.

Zheng et al. (2014) ajustaram os dados de quantidade de precipitação que foram coletados com intervalo de 5, 10 e 15 minutos utilizando os modelos de STARMA para prever as possíveis enchentes de vários locais da cidade Pequim, China. E mostrou a eficácia do modelo STARMA para ajustar dados espaço-temporais.

Clements et al. (2014) ajustaram um modelo STARMA sazonal para estimar e prever valores de NDVI (os dados do índice de vegetação diferenciada normalizada) registados por teledetecção via satélites na África Oriental.

Para a aplicação do modelo STARMA, é essencial a presença da matriz de pesos espaciais ou estrutura de vizinhanças espaciais. A especificação dos pesos espaciais é definida pelo pesquisador de uma maneira que reflete uma ordem hierárquica de vizinhos espaciais. A primeira ordem de vizinhaça é definida pelos locais mais próximos, aumentando a ordem de vizinhança conforme o aumento da distância dos vizinhos. Existem vários métodos para calcular os pesos espaciais, os mais utilizados estão apresentados no 
Capitulo 3.

Na prática, a escolha da estrutura da matriz espacial é subjetiva de acordo com a conveniência do pesquisador. Na literatura, quase não encontram-se estudos sobre a escolha de matriz de pesos para otimizar o ajustamento dos modelos STARMA.

O objetivo deste trabalho é estudar as estruturas de vizinhanças espaciais nos modelos STARMA com a finalidade de comparar os desempenhos de cada método de cálculo da matriz de pesos espaciais da classe de modelos STARMA através das simulações.

Este trabalho é dividido em 6 capítulos, descrevemos brevemente os conceitos fundamentais de séries temporais e modelos univariados de Box e Jennkins no Capítulo 2; no Capítulo 3, é introduzida a classe de modelos STARMA, suas propriedades e os ciclos iterativos da modelagem, tais como identificação, estimação, diagnóstico e previsão; são realizadas simulações para comparar diferentes estruturas de construção da matriz de pesos espaciais no Capítulo 4; no Capítulo 5 aplicamos modelos STARMA nos dados mensais do índice de preço imobiliário de venda para dois dormitórios de seis cidades metropolitanas de São Paulo, e por fim, no Capítulo 6, resumimos as conclusões do trabalho e são sugeridos alguns estudos para o futuro.

Existem vários pacotes no $\mathrm{R}$ que ajudam pesquisadores analisarem os dados geoespaciais, por exemplo SpatioTemporal, starma, Stem, spacetime, CompRandFld, spTimer, fields, geoRintro, geoR, etc. Os livros que podem ser consultados são Bivand et al. (2008) e Lamigueiro (2014). 


\section{Capítulo 2}

\section{Conceitos Básicos}

Uma série temporal é uma sequência de observações ordenadas no tempo. Uma característica importante deste tipo de dados é que as observações vizinhas são relacionadas, e o interesse é analisar e modelar esta dependência.

A modelagem de séries temporais é frequentemente usada em várias áreas de pesquisa, tais como economia, medicina, atividade agropecuária (produção agrícola), previsão de clima (temperatura) e também estudos ambientais.

A série temporal é dita ser discreta quando as observações são feitas em tempos específicos, por exemplo, valores diários de poluição na cidade de São Paulo. É considerada como contínua quando as observações são feitas continuamente no tempo, como registro de marés no porto de Santos, por exemplo.

De acordo com Morettin e Toloi (2006), há basicamente dois enfoques usados na análise de séries temporais. Em ambos, o objetivo é construir modelos para as séries, com propósitos determinados. No primeiro, a análise é feita no domínio de tempo e os modelos propostos são paramétricos (com um número finito de parâmetros). No segundo, é feita no domínio de frequência, e os modelos são não paramétricos (com um número infinito de parâmetros). E no presente estudo, trabalhamos com os modelos paramétricos no domínio de tempo.

Modelos de séries temporais são divididos em dois grupos, univariado e multivariado. Modelo univariado refere-se à modelagem de séries temporais univariadas que consistem de observações registradas sequencialmente ao longo do tempo. O modelo univariado mais conhecido é o modelo ARMA, autorregressivos e médias móveis de Box e Jenkins (1970). Modelo multivariado descreve a relação de casualidade entre várias séries temporais, por exemplo o modelo VARMA (autorregressivo e média móvel vetorial) que é a forma multivariada dos modelos univariados de Box-Jenkins ARMA.

Neste capítulo, descrevemos brevemente os conceitos fundamentais de séries temporais, como processo estocástico, estacionariedade temporal e modelo univariado ARIMA $(\mathrm{p}, \mathrm{d}, \mathrm{q})$, que serão úteis para o entendimento dos capítulos posteriores. Não pretendemos fazer uma explanação profunda, apenas uma revisão sucinta. Leitores interessados podem consultar com mais detalhe as referências oferecidas para cada assunto. Este capítulo 
está dividido principalmente em três seções: na Seção 2.1 apresentamos definições de processo estocástico, na Seção 2.2 e 2.3 apresentamos o conceito de estacionariedade temporal e os modelos univariados propostos por Box e Jenkins (1970) como AR(p), MA(q), $\operatorname{ARMA}(p, q)$, etc.

\subsection{Processo Estocástico}

Os modelos utilizados para descrever séries temporais são processos estocásticos, que são processos controlados por leis probabilísticas.

Definição 2.1. Um processo estocástico é uma família $Z=\{Z(t), t \in T\}$ e $\mathrm{Z}(\mathrm{t})$ é uma variável aleatória definida num espaço de probabilidade $(\omega, A, P)$, indexado por um parâmetro t, onde t varia no conjunto arbitrário T.

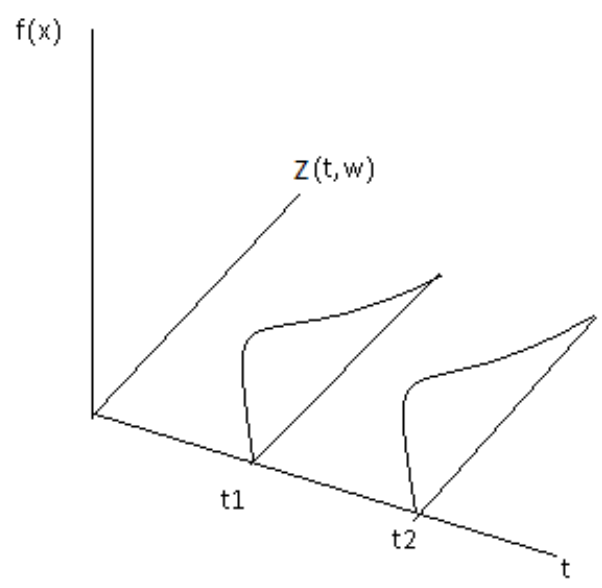

Figura 2.1: Cada variável $\mathrm{Z}(\mathrm{t})$ é definida em $(\omega, A, P)$

Como uma variável aleatória é uma função definida num espaço amostral $\Omega$. Na realidade, o processo estocástico $Z=\{Z(t), t \in T\}$ é uma função de dois argumentos $Z=\{Z(t), t \in T, \omega \in \Omega\}$.

Para cada $t \in T$, a função $\mathrm{Z}(\mathrm{t},$.$) é mensurável relativamente a A. Por outro lado,$ para cada $\omega \in \Omega$ fixado, teremos uma função $Z(., \omega)$ de $t: T \rightarrow \Re$, que é chamada trajetória, realização, função amostral do processo ou série temporal.

Uma série temporal é uma particular realização do processo estocástico, que denotamos simplesmente $\mathrm{Z}(\mathrm{t})$, onde tempo $t=1, \ldots, T$. Podemos entender um processo estocástico como uma família de trajetórias ao longo do tempo e observamos uma delas. No presente estudo, usaremos a notação vetorial $Z=\left(Z_{1}, \ldots, Z_{N}\right)^{\prime}$, ou seja, trabalharemos com séries multivariadas. 
Definição 2.2. A função distribuição n-dimensional do processo estocástico $Z=\{Z(t), t \in$ T\} é definida como:

$$
F\left(z_{1}, \ldots, z_{n} ; t_{1}, \ldots, t_{n}\right)=P\left(Z\left(t_{1}\right) \leq z_{1}, \ldots, Z\left(t_{n}\right) \leq z_{n}\right)
$$

para todo $n \geq 1$ e $t_{1}, \ldots, t_{n} \in T$.

Definição 2.3. As funções média e covariância de um processo $\mathrm{Z}(\mathrm{t})$ são dadas por:

$$
\begin{gathered}
E(Z(t))=\int_{-\infty}^{+\infty} z d F(z ; t)=\mu_{z}(t) \\
\operatorname{Cov}\left[Z\left(t_{1}\right), Z\left(t_{2}\right)\right]=\iint\left[z_{1}-\mu_{z}\left(t_{1}\right)\right]\left[z_{2}-\mu_{z}\left(t_{2}\right)\right] d F\left(z_{1}, z_{2} ; t_{1}, t_{2}\right) \\
=E\left(Z\left(t_{1}\right) Z\left(t_{2}\right)\right)-E\left(Z\left(t_{1}\right)\right) E\left(Z\left(t_{2}\right)\right) \\
=\gamma\left(t_{1}, t_{2}\right)
\end{gathered}
$$

Se $t_{1}=t_{2}=t$, temos a variância do processo:

$$
\operatorname{Var}(Z(t))=\int\left[z-\mu_{z}(t)\right]^{2} d F(z ; t)=E\left(Z^{2}(t)\right)-E^{2}(Z(t))=\gamma(t)
$$

A função de autocorrelação é definida por:

$$
\operatorname{Cor}\left(Z\left(t_{1}\right), Z\left(t_{2}\right)\right)=\rho\left(t_{1}, t_{2}\right)=\frac{\operatorname{Cov}\left(Z\left(t_{1}\right), Z\left(t_{2}\right)\right)}{\sqrt{\operatorname{Var}\left(Z\left(t_{1}\right)\right) \operatorname{Var}\left(Z\left(t_{2}\right)\right)}}
$$

\subsection{Processo Estacionário}

Segundo Morettin e Toloi (2006), um processo Z é considerado estacionário se ele se desenvolve no tempo de modo que a escolha de uma origem dos tempos não é relevante, ou seja, as características de $Z(t+\tau)$, para toda defasagem de tempo $\tau$, são as mesmas de $\mathrm{Z}(\mathrm{t})$. Teoricamente há duas formas de estacionariedade: forte (estrita) e fraca (segunda ordem).

Definição 2.4. Seja $Z=\{Z(t), t \in T\}$ um processo estocástico real com tempo discreto, de média zero e função de autocovariância denotada por $\gamma_{\tau}=E\left(Z_{t}, Z_{t+\tau}\right)$.
(a) $\gamma_{0}>0$
(b) $\gamma_{-\tau}=\gamma_{\tau}$
(c) $\left|\gamma_{\tau}\right| \leq \gamma_{0}$ 
(d) $\gamma_{\tau}$ é não negativa definida, no sentido que

$$
\sum_{j=1}^{n} \sum_{k=1}^{n} a_{j} a_{k} \gamma_{\tau_{j}-\tau_{k}}=\sum_{j=1}^{n} \sum_{k=1}^{n} a_{j} a_{k} E\left(Z_{\tau_{j}} Z_{\tau_{k}}\right) \geq 0
$$

para quaisquer números reais $a_{1}, \ldots, a_{n}$, e $\tau_{1}, \ldots, \tau_{n}$ de $\mathrm{T}$.

Definição 2.5. Um processo estocástico $Z=\{Z(t), t \in T\}$ é dito estritamente estacionário se todas as distribuições finito-dimensionais (2.1) permanencem as mesmas sob translações no tempo, ou seja:

$$
F\left(z_{1}, \ldots, z_{n} ; t_{1}+\tau, \ldots, t_{n}+\tau\right)=F\left(z_{1}, \ldots, z_{n} ; t_{1}, \ldots, t_{n}\right)
$$

para $t_{1}, \ldots, t_{n}, t_{1}+\tau, \ldots, t_{n}+\tau \in T$.

Definição 2.6. Um processo estocástico $Z=\{Z(t), t \in T\}$ é estacionário de segunda ordem, ou fracamente estacionária, ou ainda estacionário em sentido amplo se:

(a) $E(Z(t))=\mu_{z}$, constante, $\forall t \in T$

(b) $E\left(Z^{2}(t)\right)<\infty, \forall t \in T$

(c) $\operatorname{Cov}\left(Z\left(t_{1}\right), Z\left(t_{2}\right)\right)=\gamma\left(t_{1}, t_{2}\right)=g\left(\left|t_{1}-t_{2}\right|\right)$, ou seja, a covariância é função somente da defasagem $\left|t_{1}-t_{2}\right|$.

Na prática, é muito difícil usar a definição de estacionariedade estrita, tornando-se satisfatório a definição de estacionariedade de segunda ordem, a qual é atendida se a média da série é constante e se sua função de autocovariância depende apenas da defasagem de tempo entre dois pontos. Portanto a partir de agora estaremos somente interessados nesta classe de processos, que denominaremos simplesmente de processos estacionários. Note-se que se $\mathrm{Z}$ for estritamente estacionário, ele não necessitará ser fracamente estacionário, pois $E\left(Z^{2}(t)\right)<\infty, \forall t \in T$ pode não ser satisfeita.

Definição 2.7. Um processo $Z=\{Z(t), t \in T\}$ diz-se gaussiano se para qualquer subconjunto $t_{1}, \ldots, t_{n}$ de $\mathrm{T}$, o conjunto das variáveis aleatórias $\left(Z\left(t_{1}\right), \ldots, Z\left(t_{n}\right)\right)$ tem uma distribuição normal $n$-variada.

Definição 2.8. Uma sequência de variáveis aleatórias $\{\varepsilon(t), t=0, \pm 1, \ldots\}$, tal que

(a) $E(\varepsilon(t))=\mu$

(b) $\operatorname{Var}(\varepsilon(t))=\sigma^{2}$

(c) $\operatorname{Cov}\left(\varepsilon\left(t_{1}\right), \varepsilon\left(t_{2}\right)\right)=0, \forall t_{1}, t_{2} \in\{0, \pm 1, \ldots\}, t_{1} \neq t_{2}$

é chamado de ruído branco. Usualmente $\mu=0$. 
Na maioria dos casos temos séries não estacionárias, sendo necessário realizar alguma transformação até que estas se tornem estacionárias. Box e Jenkins (1970), Brockwell e Davis (2006) e Morettin e Toloi (2006) ressaltam que os principais motivos para a transformação de uma série são a estabilização da variância e a eliminação da tendência. Para estabilizar a variabilidade é indicada a raiz quadrada ou o logaritmo; e para eliminar a tendência (média não constante) é recomendada a diferenciação, que consiste em tomar diferenças sucessivas da série temporal.

Para a diferenciação da série, introduzimos uma notação de operadores, que será usada bastante nos capítulos seguintes.

(1) operador translação para o passado, denotado por B e definido por

$$
B Z_{t}=Z_{t-1}, B^{k} Z_{t}=Z_{t-k}
$$

(2) operador da diferença é definido por

$$
\triangle Z_{t}=Z_{t}-Z_{t-1}=(1-B) Z_{t}
$$

segue-se que $\triangle=1-B$.

\subsection{Modelos Box e Jenkins}

Nesta seção apresentamos brevemente o método mais utilizado na análise de modelos paramétricos que é conhecido como abordagem de Box e Jenkins (1970). O método de Box e Jenkins consiste na busca da classe de modelos autorregressivos integrados e de médias móveis, ARIMA (p,d,q). Quando uma série tiver componente sazonal (variação cíclica), temos a extensão de modelos ARIMA, o modelo SARIMA $(p, d, q) \times(P, D, Q)$.

Segundo Morettin e Toloi (2006), a construção do modelo será baseada em um ciclo iterativo que consiste nas seguintes etapas:

(1) Especificação: Um ou mais modelos da classe ARIMA são considerados para a análise;

(2) Identificação: Identificar um modelo baseando na análise de autocorrelações e autocorrelações parciais;

(3) Estimação: Estimar os parâmetros do modelo identificado;

(4) Verificação: Verificar o modelo ajustado através de uma análise de resíduos e a previsão caso o modelo seja adequado; senão voltamos à fase da identificação. Se o propósito 
é previsão, podemos escolher entre os modelos ajustados aquele que fornece o menor erro quadrático médio de previsão. Além disso, os critérios de informação AIC e BIC também podem ser empregados como método alternativo para a escolha de modelo final.

Os livros de referências básicas são Box e Jenkins (1970), Brockwell e Davis (2006), Chatfield (2016), Ehlers (2007), Wheelwright et al. (1998) e Morettin e Toloi (2006).

A classe de modelos ARIMA possui subclasses, modelos autorregressivos (AR), modelos de médias móveis (MA) e modelos autorregressivos e médias móveis (ARMA), considerando $a_{t}$ ruído branco com média zero e variância $\sigma_{a}^{2}$.

\subsubsection{Modelo Autorregressivo AR (p)}

Um processo $Z_{t}$ é o processo autorregressivo de ordem $\mathrm{p}, \mathrm{AR}(\mathrm{p})$ é definido por

$$
Z_{t}=\phi_{1} Z_{t-1}+\phi_{2} Z_{t-2}+\ldots+\phi_{p} Z_{t-p}+a_{t}
$$

Utilizando o operador translação para o passado, podemos escrever a equação (2.8) como:

$$
\left(1-\phi_{1} B-\phi_{2} B^{2}-\ldots-\phi_{p} B^{p}\right) Z_{t}=a_{t},
$$

em que $\phi(B)=1-\phi_{1} B-\phi_{2} B^{2}-\ldots-\phi_{p} B^{p}$, o operador de autorregressivo estacionário de ordem p, então temos

$$
\phi(B) Z_{t}=a_{t}
$$

\subsubsection{Modelo Média Móvel MA (q)}

Um processo $Z_{t}$ é o processo de médias móveis de ordem q, MA(q) tem a seguinte forma:

$$
Z_{t}=a_{t}-\theta_{1} a_{t-1}-\theta_{2} a_{t-2}-\ldots-\theta_{q} a_{t-q}
$$

Usando o operador de translação de tempo B, temos

$$
Z_{t}=\left(1-\theta_{1} B-\theta_{2} B^{2}-\ldots-\theta_{q} B^{q}\right) a_{t}=\theta(B) a_{t}
$$

sendo $\theta(B)=1-\theta_{1} B-\theta_{2} B^{2}-\ldots-\theta_{q} B^{q}$, um polinômio de ordem q em B.

\subsubsection{Modelo Autorregressivo e Média Móvel ARMA (p,q)}

Os modelos autorregressivos são bastante usados em várias áreas, Economia, Meteorologia, etc. É natural pensar que o valor observado no instante t depende de valores defasados do mesmo processo. Por outro lado, o modelo de média móvel como sendo um processo puramente aleatório não parece ser intuitivo e natural, principalmente para a previsão da série. Surgem, então, os modelos ARMA (p,q) que combinam as características 
dos modelos AR (p) e MA (q), sendo prático e parcimonioso.

O modelo ARMA (p,q) é dado por:

$$
Z_{t}=\phi_{1} Z_{t-1}+\ldots+\phi_{p} Z_{t-p}+a_{t}-\theta_{1} a_{t-1}-\ldots-\theta_{q} a_{t-q}
$$

o reescrito do modelo utilizando o operador de translação do tempo:

$$
\left(1-\phi_{1} B-\ldots-\phi_{p} B^{p}\right) Z_{t}=\left(1-\theta_{1} B-\ldots-\theta_{q} B^{q}\right) a_{t},
$$

ou seja $\phi(B) Z_{t}=\theta(B) a_{t}$.

No capítulo 4, apresentaremos a classe de modelos STARMA, que é uma extensão da classe de modelos ARMA a séries multivariadas no espaço.

\subsubsection{Modelo ARIMA (p,d,q)}

Na prática, muitas séries encontradas são não estacionárias por ter componente de tendência e média não constante. Os modelos descritos até agora são apropriados somente para séries estacionárias. Para as séries não estacionárias e se tornarem estacionárias depois das diferenças das séries, o modelo ARIMA é aplicável.

Seja $W_{t}$ estacionária, d-ésima diferença de série $Z_{t}$ :

$$
W_{t}=\triangle^{d} Z_{t},
$$

e com o operador de translação de tempo B, temos:

$$
W_{t}=\triangle^{d} Z_{t}=(1-B)^{d} Z_{t}
$$

Ajustando um modelo ARMA (p,q) para $W_{t}$ :

$$
W_{t}=\phi_{1} W_{t-1}+\ldots+\phi_{p} W_{t-p}+a_{t}-\theta_{1} a_{t-1}-\ldots-\theta_{q} a_{t-q}
$$

que é equivalente a um modelo $\operatorname{ARIMA}(\mathrm{p}, \mathrm{d}, \mathrm{q})$ para $Z_{t}$ :

$$
\begin{gathered}
\triangle^{d} Z_{t}=\phi_{1} \triangle^{d} Z_{t-1}+\ldots+\phi_{p} \triangle^{d} Z_{t-p}+a_{t}-\theta_{1} a_{t-1}-\ldots-\theta_{q} a_{t-q} \\
\left(1-\phi_{1} B-\ldots-\phi_{p} B^{p}\right) \triangle^{d} Z_{t}=\left(1-\theta_{1} B-\ldots-\theta_{q} B^{q}\right) a_{t} \\
\phi(B) \triangle^{d} Z_{t}=\theta(B) a_{t} \\
\phi(B)(1-B)^{d} Z_{t}=\theta(B) a_{t} .
\end{gathered}
$$




\subsubsection{Modelo SARIMA $(\mathrm{p}, \mathrm{d}, \mathrm{q}) \times(\mathrm{P}, \mathrm{D}, \mathrm{Q})$}

Muitas séries além de terem uma tendência, também possuem um componente sazonal, isto é, uma variação cíclica que se repete por intervalos iguas de tempo, ou seja, a cada k observações.

Os principais tipos de sazonalidade são divididos em determinística e estocástica. Se a sazonalidade é estritamente periódica (determinística), isto é, a função componente sazonal $\mu_{t}$ do processo $Z_{t}$ satisfaz $\mu_{t}-\mu_{t-k}=0$; ao contrário da estocástica, $\mu_{t}-\mu_{t-k}=Y_{t}$, em que $Y_{t}$ é um processo estacionário.

Para tornar uma série estacionária, estimamos primeiro o componente sazonal e depois subtraí-lo da série original $Z_{t}$. Existem várias técnicas para ajustar componente sazonal, por exemplo, o método de regressão usando funções de seno e cosseno para sazonalidade determinística; o método de médias móveis para sazonalidade estocástica. A maneira frequentemente utilizada para eliminar as sazonalidades determinística e estocástica é aplicar as diferenças sazonais das séries, denotada por $\left(1-B^{k}\right)^{D} Z_{t}$ ou $\triangle_{k}^{D} Z_{t}$, $\mathrm{D}$ a quantidade de diferenças. (Morettin e Toloi (2006))

Definimos o modelo SARIMA $(\mathrm{p}, \mathrm{d}, \mathrm{q}) \times(\mathrm{P}, \mathrm{D}, \mathrm{Q})$ como

$$
\phi(B) \Phi\left(B^{k}\right) \triangle^{d} \triangle_{k}^{D} Z_{t}=\theta(B) \Theta\left(B^{k}\right) a_{t}
$$

que é equivalente a

$$
\phi(B) \Phi\left(B^{k}\right)(1-B)^{d}\left(1-B^{k}\right)^{D} Z_{t}=\theta(B) \Theta\left(B^{k}\right) a_{t},
$$

em que

$\phi(B)=1-\phi_{1} B-\phi_{2} B^{2}-\ldots-\phi_{p} B^{p}$,

$\Phi\left(B^{k}\right)=1-\Phi_{1} B^{k}-\Phi_{2} B^{2 k}-\ldots-\Phi_{P} B^{P k}$,

$\theta(B)=1-\theta_{1} B-\theta_{2} B^{2}-\ldots-\theta_{q} B^{q}$,

$\Theta\left(B^{k}\right)=1-\Theta_{1} B^{k}-\Theta_{2} B^{2 k}-\ldots-\Theta_{Q} B^{Q k}$.

\subsection{Processos Iterativos}

Como mencionamos na introdução da seção 2.3 , os estágios do ciclo iterativo do método de Box e Jenkins são a identificação, a estimação e a verificação, na medida em que especificamos a classe geral de modelos ARIMA. Nesta seção descrevemos brevemente essas três fases, e servirá como conhecimento fundamental para o entendimento do ciclo iterativo do STARMA no Capítulo 3. 


\subsubsection{Identificação}

A fase de identificação é feita principalmente com base nas funções estimadas de autocorrelação e autocorrelação parcial.

A função autocorrelação fac $\rho_{i}$ estimada é definida por

$$
r_{i}=\frac{c_{i}}{c_{0}}, i=0,1, \ldots, T-1
$$

$\operatorname{com} c_{i}$ a estimativa da função de autocovariância,

$$
c_{i}=\frac{1}{T} \sum_{t=1}^{T-i}\left[\left(Z_{t}-\bar{Z}\right)\left(Z_{t+i}-\bar{Z}\right)\right], i=0,1, \ldots, T-1,
$$

em que $\bar{Z}=\frac{1}{T} \sum_{t=1}^{T} Z_{t}$. Recordando que $c_{i}=c_{-i}$ e $r_{i}=r_{-i}$.

Segundo Box et al. (1994), uma outra ferramenta utilizada para facilitar o processo de identificação é a função de autocorrelação parcial facp. Esta medida corresponde a correlação de $Z_{t}$ e $Z_{t-i}$ removendo o efeito das observações $Z_{t-1}, \ldots, Z_{t-i+1}$ e é denotada por $\phi_{k k}$.

Um método geral para encontrar a facp para um processo estacionário com fac $\rho_{i}$ é utilizando as equações de Yule-Walker

$$
\left[\begin{array}{rrrrr}
1 & \rho_{1} & \rho_{2} & \ldots & \rho_{k-1} \\
\rho_{1} & 1 & \rho_{1} & \ldots & \rho_{k-2} \\
\cdot & & & & \\
\cdot & & & & \\
\cdot & & & & \\
\rho_{k-1} & \rho_{k-2} & \rho_{k-3} & \ldots & 1
\end{array}\right] \quad\left[\begin{array}{c}
\phi_{k 1} \\
\phi_{k 2} \\
\ldots \\
\phi_{k k}
\end{array}\right]=\left[\begin{array}{c}
\rho_{1} \\
\rho_{2} \\
\ldots \\
\rho_{k}
\end{array}\right]
$$

Resolvendo as equações acima sucessivamente para $\mathrm{k}=1,2, \ldots$, obtemos $\phi_{k k}$ da seguinte maneira

$$
\begin{gathered}
\phi_{11}=\rho_{1}, \\
\phi_{22}=\frac{\left|\begin{array}{rr}
1 & \rho_{1} \\
\rho_{1} & \rho_{2}
\end{array}\right|}{\left|\begin{array}{rr}
1 & \rho_{1} \\
\rho_{1} & 1
\end{array}\right|}=\frac{\rho_{2}-\rho_{1}^{2}}{1-\rho_{1}^{2}},
\end{gathered}
$$




$$
\phi_{33}=\frac{\left|\begin{array}{rrr}
1 & \rho_{1} & \rho_{1} \\
\rho_{1} & 1 & \rho_{2} \\
\rho_{2} & \rho_{1} & \rho_{3}
\end{array}\right|}{\left|\begin{array}{rrr}
1 & \rho_{1} & \rho_{2} \\
\rho_{1} & 1 & \rho_{1} \\
\rho_{2} & \rho_{1} & 1
\end{array}\right|}=\frac{\rho_{3}+\rho_{2}^{2} \rho_{1}+\rho_{1}^{3}-2 \rho_{1} \rho_{2}-\rho_{1}^{2} \rho_{3}}{1-2 \rho_{1}^{2}-\rho_{2}^{2}}
$$

em geral temos $\phi_{k k}=\frac{\left|\mathbf{P}_{\mathbf{k}}^{*}\right|}{\left|\mathbf{P}_{\mathbf{k}}\right|}$, onde $P_{k}$ é a matriz de autocorrelação, e $P_{k}^{*}$ é a matriz $P_{k}$ com a última coluna substituída pelo vetor de autocorrelação. Lembremos que para calcular as autocorrelações parciais estimadas é só substituir as autocorrelações $\rho_{i}$ pelas suas estimativas $r_{i}$. Mais detalhes sobre a construção de autocorrelação parcial podem ser encontradas em Box et al. (1994) e Morettin e Toloi (2006).

O procedimento de identificação consiste em três partes:

(1) Verificar se há necessidade de uma transformação na série original com a finalidade de estabilizar sua variância, por exemplo, usando logaritmo neperiano;

(2) Tomamos diferenças d vezes necessárias da série obtida no item (1) com o objetivo de tornar o processo estacionário, de forma que $\triangle^{d} Z_{t}$ seja reduzido para a um $\operatorname{ARMA}(\mathrm{p}, \mathrm{q})$. O número de diferenças d é alcançado quando a função de autocorrelação amostral da série $\triangle^{d} Z_{t}$ decresce rapidamente para zero;

(3)Identificar as ordens do modelo ARMA (p,q). A ordem autorregressivo p é determinada pela função de autocorrelação parcial facp e a ordem de média móvel q é determinada pela fac função de autocorrelação.

Conforme Brockwell e Davis (2006), Ehlers (2007) e Morettin e Toloi (2006), os processos $\operatorname{AR}(p), M A(q)$ e $\operatorname{ARMA}(p, q)$ apresentam fac com seguintes características:

(1) fac de um processo $\mathrm{AR}(\mathrm{p})$ decai exponencialmente e/ou com senóides amortecidas, infinita em extensão;

(2) fac de um processo MA (q) é igual a zero para lags (defasagens de tempo) maiores do que q;

(3) fac de um processo ARMA (p,q) é infinita em extensão, e decai exponencialmente e/ou com senóides amortecidas depois do "lag"maior que q.

E para a função de autocorrelação parcial facp, temos as características: 
(1) um processo AR (p) tem facp zero para lags maiores do que p;

(2) um processo MA (q) tem facp que decai exponencialmente e/ou com senóides amortecidas, infinita em extensão;

(3) o comportamento de facp de um processo ARMA (p,q) é igual a facp de um processo MA.

Resumimos os comportamentos teóricos de fac e facp para as menores ordens dos processos $\operatorname{AR}(\mathrm{p}), \mathrm{MA}(\mathrm{q})$ e $\operatorname{ARMA}(\mathrm{p}, \mathrm{q})$ na Tabela 2.1.

\begin{tabular}{c|l|l}
\hline Modelo & \multicolumn{1}{|c|}{ FAC $\boldsymbol{\rho}_{\boldsymbol{k}}$} & \multicolumn{1}{c}{ FACP $\boldsymbol{\phi}_{\boldsymbol{k} \boldsymbol{k}}$} \\
\hline $\mathrm{AR}(1)$ & decai exponencialmente & somente $\phi_{11} \neq 0$ \\
\hline $\operatorname{AR}(2)$ & $\begin{array}{l}\text { mistura de exponenci- } \\
\text { ais e/ou com senóides } \\
\text { amortecidas }\end{array}$ & somente $\phi_{11} \neq 0$ e $\phi_{22} \neq 0$ \\
\hline $\mathrm{MA}(1)$ & somente $\rho_{1} \neq 0$ & decai exponencialmente \\
\hline $\mathrm{MA}(2)$ & somente $\rho_{1} \neq 0$ e $\rho_{2} \neq 0$ & $\begin{array}{l}\text { mistura de exponenciais ou } \\
\text { senóides amortecidas }\end{array}$ \\
\hline $\operatorname{ARMA}(1,1)$ & $\begin{array}{l}\text { decai exponencialmente de- } \\
\text { pois do lag 1 }\end{array}$ & $\begin{array}{l}\text { dominada por decaimento } \\
\text { exponencial depois do lag 1 }\end{array}$ \\
\hline
\end{tabular}

Tabela 2.1: Comportamento das fac e facp de um processo ARMA(p,q)

Para modelo SARIMA $(\mathrm{p}, \mathrm{d}, \mathrm{q}) \times(\mathrm{P}, \mathrm{D}, \mathrm{Q})_{s}$, não há, em princípio, nenhuma dificuldade adicional na identificação. O número $\mathrm{D}$ de diferença sazonal é alcançado quando a série fica estacionária sem o componente sazonal, na maioria das vezes, assumem valores no máximo iguais a 2. A ordem autorregressivo $\mathrm{P}$ e a ordem de média móvel $\mathrm{Q}$ de modelo SARIMA são determinadas baseando nas fac e facp da série diferenciada nos lags s, $2 \mathrm{~s}, 3 \mathrm{~s}, \ldots$, que são equivalentes aos lags 1,2,3...quando obtemos valores de p e q de modelo ARIMA.

A forma alternativa de identificação é utilizar os critérios de informação que avalia as qualidades de ajustes através de penalizar a inclusão de parâmetros extras. Os critérios mais utilizados são AIC (Critério de Informação de Akaike) e BIC (Critério de Informação Bayesiano). A regra consiste em selecionar o modelo cujo critério de informação seja mínimo. AIC e BIC são calculados como:

$$
\begin{gathered}
A I C(k, l)=-2 \ln \left(\hat{\sigma}_{n}^{2}\right)+2(n+2), \\
B I C(k, l)=-2 \ln \left(\hat{\sigma}_{n}^{2}\right)+n \frac{\ln T}{T},
\end{gathered}
$$

em que T é o número de observações, n é o número de parâmetros e $\hat{\sigma}_{n}^{2}$ é o estimador de máxima verossimilhança da variância residual do modelo. 


\subsubsection{Estimação}

Despois de ter identificado um modelo provisório para uma série, estimamos os seus parâmetros. Existem vários métodos de estimação, como método de momentos, mínimos quadrados e máxima verossimilhança, Morettin e Toloi (2006).

O método de momento é um método para estimar os parâmetros populacionais, baseado em derivar as equações que relacionam os momentos da população (isto é, os valores esperados de potências da variável aleatória em questão) para os parâmetros de interesse. As equações são resolvidas para os parâmetros de interesse utilizando os momentos de amostra no lugar dos momentos populacionais que são desconhecidos. Isso resulta em estimativas desses parâmetros. Estimadores obtidos pelo método dos momentos não têm propriedades boas, quando comparadas com os demias. O método de momentos foi introduzido por Karl Pearson em 1894. Mais detalhes vejam Fuller (2009). A estimação de mínimos quadrados também é conhecida como mínimos quadrados ordinários, que minimiza a soma dos resíduos quadrados, partindo de um sistema de equações não lineares. O método foi criado por Carl Friedrich Gauss (1795). E por último, o método de máxima verssimilhança estima os parâmetros de interesse com os dados observados maximizando a função de verossimilhança (função densidade conjunta), sendo o que resulta em melhores estimativas.

Para um modelo ARIMA(p,d,q), temos $\mathrm{p}+\mathrm{q}+1$ parâmetros para estimar. Seja vetor $\boldsymbol{\xi}=\left(\boldsymbol{\phi}, \boldsymbol{\theta}, \sigma_{a}^{2}\right)$, em que $\boldsymbol{\phi}=\left(\phi_{1}, \ldots, \phi_{p}\right)$ e $\boldsymbol{\theta}=\left(\theta_{1}, \ldots, \theta_{q}\right)$. Dadas n observações $Z_{1}, \ldots, Z_{n}$, consideramos a função de verossimilhança $L\left(\xi \mid Z_{1}, \ldots, Z_{n}\right)$ como uma função de $\xi$, então queremos encontrar os valores de $\xi$ que maximizam $L$ ou $l=\log (L)$.

Para determinar o estimador máxima verossimilhança (EMV), trabalharemos com a suposição que o processo $a_{t}$ é normal, ou seja, para cada t, temos $a_{t} \sim N\left(0, \sigma_{a}^{2}\right)$, nestas considerações os EMV serão aproximadamente igauis aos estimadores de mínimos quadrados (EMQ).

Tomamos d diferenças na série para alcançarmos estacionariedade, ficamos $\operatorname{com} n=$ $\mathrm{N}$ - d observações denotadas por $W_{1}, \ldots, W_{n}$, onde $W_{t}=\Delta^{d} Z_{t}$, resultando em um modelo $\operatorname{ARMA}(p, q)$ estacionário, então escrevemos

$$
a_{t}=W-\phi_{t} W_{t-1}-\cdots-\phi_{p} W_{t-p}+\theta_{1} a_{t-1}+\cdots+\theta_{q} a_{t-q}
$$

- Para calcular os $a_{t}$ é necessário obter valores iniciais para os W's e os a's. Podemos resolver esta questão através de dois métodos: condicional, que os valores iniciais desconhecidos são substituídos por valores que supomos serem razoáveis; incondicional, em que o valores iniciais são estimados utilizando um precedimento denominado "backforecasting". Em nosso estudo, somente focamos em método de máxima verssimilhança condicional. Mais detalhes sobre incondicional podem ser encontrados no Morettin e Toloi (2006) e Box et al. (1994). 
Sob a suposição de $a_{t} \sim N\left(0, \sigma_{a}^{2}\right)$ temos que a função de densidade conjunta de $a_{1}, \ldots, a_{n}$ :

$$
f\left(a_{1}, \ldots, a_{n}\right)=(2 \pi)^{\frac{-n}{2}}\left(\sigma_{a}^{2}\right)^{\frac{-n}{2}} \exp \left\{-\sum_{t=1}^{n} \frac{a_{t}^{2}}{2 \sigma_{a}^{2}}\right\} .
$$

Suponha que são dados p valores $W_{t}$ e q valores $a_{t}$, denotados por $W_{t}^{*}$ e $a_{t}^{*}$. A função de verossimilhança condicional é dada por

$$
\begin{gathered}
L\left(\boldsymbol{\xi} \mid \boldsymbol{W}, \boldsymbol{W}^{*}, \boldsymbol{a}^{*}\right)=(2 \pi)^{\frac{-n}{2}}\left(\sigma_{a}\right)^{-n} \exp \left\{-\frac{1}{2 \sigma_{a}^{2}} \sum_{t=1}^{n}\left(W_{t}-\phi_{1} W_{t-1}-\ldots-\right.\right. \\
\left.\left.\phi_{p} W_{t-p}+\theta_{1} a_{t-1}+\ldots+\theta_{q} a_{t-q}\right)^{2}\right\} .
\end{gathered}
$$

e tomando-se o logariitmo de L, obtém-se:

$$
l\left(\boldsymbol{\xi} \mid \boldsymbol{W}, \boldsymbol{W}^{*}, \boldsymbol{a}^{*}\right) \propto-n \log \sigma_{a}-\frac{S\left(\boldsymbol{\eta} \mid \boldsymbol{W}, \boldsymbol{W}^{*}, \boldsymbol{a}^{*}\right)}{2 \sigma_{a}^{2}},
$$

em que:

$$
S\left(\boldsymbol{\eta} \mid \boldsymbol{W}, \boldsymbol{W}^{*}, \boldsymbol{a}^{*}\right)=\sum_{t=1}^{n} a_{t}^{2}\left(\boldsymbol{\eta} \mid \boldsymbol{W}, \boldsymbol{W}^{*}, \boldsymbol{a}^{*}\right)
$$

que é chamado de soma de quadrados condicional residual.

Seja $l_{*}(\boldsymbol{\xi})=l\left(\boldsymbol{\xi} \mid \boldsymbol{W}, \boldsymbol{W}^{*}, \boldsymbol{a}^{*}\right)$ e $S_{*}(\boldsymbol{\eta})=S\left(\boldsymbol{\eta} \mid \boldsymbol{W}, \boldsymbol{W}^{*}, \boldsymbol{a}^{*}\right)$. Para maximizar $l_{*}(\boldsymbol{\xi})$, isso é equivalente a minimizar $S_{*}(\boldsymbol{\eta})$, e a escolha dos valores $\mathbf{W}^{*}$ e $\mathbf{a}^{*}$ é realizado propondo os elementos destes vetores iguais às suas esperanças $E\left(a_{t}\right)=0$, o mesmo ocorrendo com os elementos de $\mathbf{W}^{*}$; se $E\left(W_{t}\right) \neq 0$, deve-se subsituir $\mathbf{W}^{*}$ por $\bar{W}$.

\subsubsection{Diagnóstico}

Depois de identificar e estimar o modelo, precisamos verificar se o modelo representa adequadamente os dados, isso pode ser feito através de análise de resíduos, tanto pelo gráfico de FAC ou pelo teste como Ljung e Box (1978), etc. Caso tiver qualquer insuficiência revelada pode sugerir um modelo alternativo como sendo adequado, e assim volta no início do cíclo iterativo de Box e Jenkins.

Quando o modelo for adequado, esperamos que os resíduos estimados ou simplesmente resíduos sejam ruídos brancos, ou seja, se distribuem de forma aleatória em torno de zero com variância constante e sejam não correlacionados. Para verificar se os resíduos não são correlacionados, podemos observar pelo gráfico de FAC, que apresenta as autocorrelações estimadas não significativas, iguais a zero (nenhum lag fora dos intervalos de confiança). E será também utilizado, o teste de Ljung e Box, cujas hipóteses são:

$$
H_{0}: \rho_{1}=\ldots=\rho_{m}=0
$$




$$
H_{1}: \rho_{k} \neq 0, \forall k \in\{1, \ldots, m\}
$$

e sendo a estatística de teste

$$
Q(m)=n(n+2) \sum_{j=1}^{m} \frac{\hat{r}_{j}^{2}}{(n-j)} \stackrel{H_{0}}{\sim} \chi_{m-p-q}^{2}
$$

Note que o número m de autocorrelações amostrais $\hat{r}_{j}$ é escolhido subjetivamente, em geral entre 15 e 20.

Como para a estimação de máxima verossimilhança supomos que os resíduos tenham distribuição normal, então precisamos também verificar a normalidade dos resíduos. Neste caso, podemos usar o teste de Shapiro e Wilk (1965) ou Bera e Jarque (1981), em que a hipótese nula é a normalidade dos resíduos versus a hipótese alternativa de não normalidade.

\subsubsection{Previsão}

Utilizar o modelo de séries identificado e estimado para fazer previsões é um dos principais objetivos da análise de séries temporais. O nosso interesse é exatamente prever os valores de $Z_{T+h}, h \geq 1$, supondo que temos as observações $Z_{1}, Z_{2}, \ldots, Z_{T}$, até o momento $\mathrm{T}$, que é chamado de origem das previsões, e h horizonte ou previsão $h$ passos a frente. Denotamos a previsão de origem $\mathrm{T}$ e horizonte h como $\hat{Z}_{T}(h)$. Para Brockwell e Davis (2006) e Montgomery et al. (1990), a diferença entre os valores observados e previstos $Z_{T+h}-\hat{Z}_{T}(h)$ é chamada de erro de previsão escrito por $e_{T}(h)$.

Para a previsão assumiremos que a série $W_{t}=(1-B)^{d} Z_{t}$ é estacionária e os parâmetros do modelo conhecida. Segundo Morettin e Toloi (2006), a previsão $\hat{Z}_{t}(h)$ pode ser escrita nas três formas básicas que são a equação de diferença, choques aleatórios e forma invertidas. Neste estudo, adotamos a forma de diferença (2.23) para realizar a previsão.

$$
Z_{t+h}=\phi_{1} Z_{t+h-1}+\ldots+\phi_{p+d} Z_{t+h-p-d}-\theta_{1} a_{t+h-1}-\ldots-\theta_{q} a_{t+h-q}+\left[a_{t+h}\right]
$$

Aplicamos a esperança condicional na equação 2.23, temos:

$$
\begin{aligned}
\hat{Z}_{t}(h) & =E\left(\phi_{1} Z_{t+h-1}+\ldots+\phi_{p+d} Z_{t+h-p-d}-\theta_{1} a_{t+h-1}-\ldots-\theta_{q} a_{t+h-q}+a_{t+h} \mid Z_{t}, Z_{t-1}, \ldots\right) \\
& =\phi_{1}\left[Z_{t+h-1}\right]+\ldots+\phi_{p+d}\left[Z_{t+h-p-d}\right]-\theta_{1}\left[a_{t+h-1}\right]-\ldots-\theta_{q}\left[a_{t+h-q}\right]+a_{t+h} .
\end{aligned}
$$

em que

(a) $\left[Z_{t+j}\right]=E\left(Z_{t+j} \mid Z_{t}, Z_{t-1}, \ldots\right)=\left\{\begin{array}{ccc}Z_{t+j}, & \text { quando } & j \leq 0 \\ \hat{Z}_{t}(j), & \text { quando } & j>0\end{array}\right.$. 
(b) $\left[a_{t+j}\right]=E\left(a_{t+j} \mid Z_{t}, Z_{t-1}, \ldots\right)=\left\{\begin{array}{lll}a_{t+j}, & \text { quando } & j \leq 0 \\ 0, & \text { quando } & j>0\end{array}\right.$. 


\section{Capítulo 3}

\section{Modelos STARMA}

No capítulo 2, introduzimos a classe de modelo ARIMA, que é um dos tipos de modelo mais utilizado para ajustar um conjunto de dados históricos de uma única série de interesse.

Além dos modelos univariados como ARMA, existem outros modelos multivariados que tentam descrever e prever várias séries temporais simultaneamente. Os modelos mais conhecidos são modelos VAR (Autorregressivo Vetorial) e VARMA (Autorregressivo e Média Móvel Vetorial), por serem bastante utilizados em estudo das correlações entre distintas variáveis macroeconômicas. Mais detalhes se encontram em Morettin (2008), Tsay (2005) e Tsay (2013).

Neste capítulo, será apresentada a classe de modelos STARMA (Autorregressivo e Média Móvel Espaço-temporal), que é um caso especial do modelo VARMA. O modelo STARMA é utilizado para descrever dados de séries temporais espacialmente localizados, ele é caracterizado pela dependência linear defasada tanto no espaço quanto no tempo. Os processos da modelagem são caracterizados por um conjunto de dados observados ao longo do tempo em diversas regiões (dizemos em $\mathrm{N}$ regiões), em que as dependências das $\mathrm{N}$ séries são relacionadas sistematicamente com a localização das regiões de estudo, além da existência de correlação serial dentro de cada região.

As N séries são modeladas simultaneamente através de uma combinação linear das observações anteriores, levando-se em consideração as suas dependências geográficas com os seus vizinhos (regiões de vizinhança). Diferente dos modelos univariados que consideram somente as influências temporais, o modelo STARMA além de considerar que o passado recente tem uma influência maior do que o passado distante, as regiões de vizinhança mais pr]oximas também exercem uma influência maior do que os vizinhos mais distantes; e essa dependência inter-locais é definida por uma matriz de distância, dando os pesos maiores aos vizinhos mais proximos.

A classe de modelos STARMA comecou a aparecer na década 70 nos artigos de Cliff e Ord (1975), Martin e Oeppen (1975), e nos anos de 1980, foi mais desenvolvida e aprimorada principalmente por Pfeifer e Deutsch. Segundo Pfeifer e Deutsch (1980a) como os modelos univariados de Box e Jenkins (1970), a metodologia de aplicação dos 
modelos STARMA também consiste nos ciclos iterativos de identificação, estimação e diagnóstico do modelo selecionado. Após 1981, os modelos STARMA passaram por um período de esquecimento devido às dificuldades de implementação computacional, eles somente foram resgatados, e até sendo bastante utilizados nos útimos anos em decorrência de grande desenvolvimento tecnológico.

\subsection{Matriz de Peso Espacial $\mathbf{W}^{(l)}$}

Os processos de modelagem da classe de modelos STARMA são descritos pelas variáveis aleatórias $Z_{i}(t)$, seja $Z_{i}(t)$ a variável de interesse no local i e no tempo t, com $\mathrm{i}=1,2, \ldots, \mathrm{N}$ e $\mathrm{t}=1,2, \ldots, \mathrm{T}$, sendo que temos dependências geográficas entre $\mathrm{N}$ locais de estudo. Para a utilização do modelo STARMA, além da suposição de estacionariedade temporal, também temos a estacionariedade espacial, isto é, a dependência entre as observações de cada região e as observações de regiões vizinhas têm que ser a mesma para todos os locais, segundo Pfeifer e Deutsch (1980a).

Para construir a forma de um modelo STARMA que será apresentado na seção 3.2, é necessário definir primeiro o operador de defasagem espacial $L^{(l)}$. Seja $L^{(l)}$ a defasagem espacial da l-ésima ordem ou vizinhanças de l-ésima ordem. Temos

$$
\begin{gathered}
L^{(0)} Z_{i}(t)=Z_{i}(t), \\
L^{(l)} Z_{i}(t)=\sum_{j=1}^{N} w_{i j}^{(l)} Z_{j}(t), \quad l>0,
\end{gathered}
$$

em que $w_{i j}^{(l)}$ são os pesos com

$$
\sum_{j=1}^{N} w_{i j}^{(l)}=1
$$

para o local i, e $w_{i j}^{(l)}$ diferentes de zero se o local $\mathrm{j}(\mathrm{j}=1,2, \ldots, \mathrm{N})$ é o local de vizinhança da l-ésima ordem do local i.

A matriz $\mathbf{W}^{(l)}$ de dimensão $\mathrm{N} \times \mathrm{N}$ com cada linha somando um é a forma matricial dos pesos $w_{i j}^{(l)}$. Seja $\mathbf{Z}(t)$, o vetor coluna de $\mathrm{N} \times 1$ das variáveis $Z_{i}(t)$, então

$$
\begin{gathered}
L^{(0)} \mathbf{Z}(t)=\mathbf{W}^{(0)} \mathbf{Z}(t)=\mathbf{I}_{N \times N} \mathbf{Z}(t), \\
L^{(l)} \mathbf{Z}(t)=\mathbf{W}^{(l)} \mathbf{Z}(t), \quad l>0 .
\end{gathered}
$$

De acordo com Pfeifer e Deutsch (1980a), a especificação dos pesos $w_{i j}^{(l)}$ é definido pelo pesquisador da forma que reflete uma ordem hierárquica de vizinhos espaciais. A primeira ordem de vizinhaça é formada pelos locais mais próximos; vizinhos de segunda ordem são os vizinhos mais distantes do que vizinhos da primeira ordem; e assim por 
diante, aumentando a ordem de vizinhança conforme a distancia dos vizinhos. Na Figura 3.1 são apresentadas as quatro primeiras ordens de vizinhos espaciais em duas dimensões desenvolvidas por Pfeifer e Deutsch (1980a), ou seja, neste caso temos as quatros matrizes $\mathbf{W}^{(1)}, \mathbf{W}^{(2)}, \mathbf{W}^{(3)}$ e $\mathbf{W}^{(4)}$.

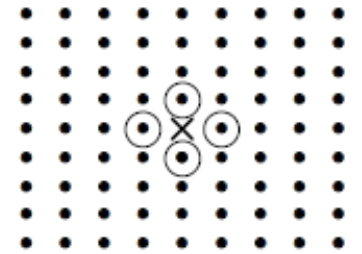

Ordem 1

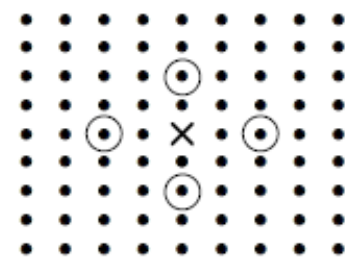

Ordem 3

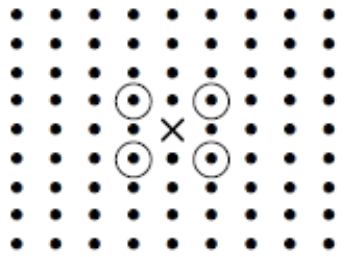

Ordem 2

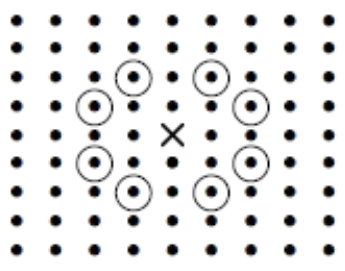

Ordem 4

Figura 3.1: Ordem de vizinhança. Pfeifer e Deutsch (1980a), Biz (2014)

Existem várias maneiras para calcular os pesos da matriz de vizinhança $\mathbf{W}^{(l)}$, as mais utilizadas são contiguidade espacial (Figura 3.1), função potência da distância, e função exponencial da distância, veja Pfeifer e Deutsch (1980a), Getis (2009), Smith (2013), Rao e Antunes (2004) e Martins (2013).

No caso de contiguidade espacial, várias ordens de matriz de pesos $(l>0)$ podem ser adotadas, o número de ordem é definido pelo pesquisador e os pesos $w_{i j}^{(l)}$ para l-ésima ordem são definidos da seguinte forma:

$$
w_{i j}^{(l)}=\left\{\begin{array}{lll}
\frac{1}{n_{i}^{(l)}} & , \quad \text { para o local } \mathrm{j} \text { que pertence à } l \text {-ésima ordem do local i } \\
0, & \text { c.c }
\end{array}\right.
$$

em que $n_{i}^{(l)}$ é o número de vizinhos da ordem $l$ do local i, satisfazendo a equação 3.1.

Podemos perceber que quando se utiliza o método de contiguidade espacial, a escolha da ordem de vizinhança e o número de locais que pertencem a cada ordem é subjetivo, depende da habilidade do pesquisador. Para evitar essa subjetividade na escolha da matriz de pesos, Rao e Antunes (2004), Martins (2013) e Smith (2013) sugeriram formas alternativas de obter os pesos usando função de potência ou exponencial da distância entre dois locais, neste caso, a maior ordem de vizinhança é 1, ou seja, somente uma matriz de $\mathbf{W}^{(l)}$ será requerida. 
Pelo método da função potência da distância, temos os cálculos de pesos:

$$
w_{i j}^{(l)}= \begin{cases}\frac{d_{i j}^{-\alpha}}{\sum_{k \neq i}^{N} d_{i k}^{-\alpha}}, & \mathrm{i} \neq \mathrm{j}, \mathrm{j}=1, \ldots, N, \\ 0 & \mathrm{i}=\mathrm{j},\end{cases}
$$

em que $d_{i j}$ é a distância entre local i e j, $\alpha$ é qualquer exponente positivo, em geral $\alpha=1$ ou 2 , e $\sum_{j=1}^{N} w_{i j}^{(l)}=1$.

Temos os pesos espaciais calculados pelo método da função exponencial da distância $d_{i j}$ :

$$
w_{i j}^{(l)}= \begin{cases}\frac{e^{-\alpha d_{i j}}}{\sum_{k \neq i}^{N} e^{-\alpha d_{i k}}}, & \mathrm{i} \neq \mathrm{j}, \mathrm{j}=1, \ldots, N, \\ 0 & \mathrm{i}=\mathrm{j},\end{cases}
$$

em que $d_{i j}$ é a distância entre local i e j, $\alpha$ é qualquer exponente positivo e $\sum_{j=1}^{N} w_{i j}^{(l)}=1$.

Uma das maneira mais conhecida para calcular a distância reta entre dois locais é a euclidiana.

Seja $d_{i j}$ a distância euclidiana entre dois pontos $\left(a_{i}, b_{i}\right)$ e $\left(a_{j}, b_{j}\right)$, temos

$$
d_{i j}=\sqrt{\left(a_{i}-a_{j}\right)^{2}+\left(b_{i}-b_{j}\right)^{2}}
$$

em que $a_{i}$ e $b_{i}$ são latitude e longitude convertidas em UTM (Universal Transverso de Mercator) do local i, assim como $a_{j}$ e $b_{j}$ são latitude e longitude convertidas em UTM do local j. O sistema UTM é usado para corrigir a projeção cilíndrica da Terra, transformando-a em uma projeção plana.

Para duas localidades próximas, calcular a distância euclidiana pode ser uma excelente aproximação. Porém, para pontos distantes, é melhor levar em consideração no cálculo da distância a curvatura da Terra. E neste trabalho, apresentamos mais uma maneira para calcular a distância, a distância de Grande Círculo ou Ortodromia.

Seja $L_{i}=\left(\theta_{i}, \lambda_{i}\right)$ e $L_{j}=\left(\theta_{j}, \lambda_{j}\right)$ dois pontos na superfície da Terra, em que $\theta_{k}$ e $\lambda_{k}$, $k=i, j$, representam a latitude e a longitude do local i e $\mathrm{j}$, respectivamente. A distância $d_{i j}$ entre $L_{i}$ e $L_{j}$ é dada por

$$
d_{i j}=\left\|L_{i}-L_{j}\right\|=R \phi
$$

em que $\mathrm{R}$ é o raio da Terra e $\phi$ satisfaz

$$
\cos \phi=\sin \theta_{i} \sin \theta_{j}+\cos \theta_{i} \cos \theta_{j} \cos \left(\lambda_{i}-\lambda_{j}\right)
$$

Mais detalhes e demonstrações podem ser encontrados em Sassi $(2016)$. No nosso trabalho, adotamos essa distância para calcular os pesos $w_{i j}^{(l)}, l=1$. 


\subsection{Modelos STARMA}

O modelo STARMA é definido por

$$
Z_{i}(t)=\sum_{k=1}^{p} \sum_{l=0}^{\lambda_{k}} \phi_{k l} L^{(l)} Z_{i}(t-k)-\sum_{k=1}^{q} \sum_{l=0}^{m_{k}} \theta_{k l} L^{(l)} \epsilon_{i}(t-k)+\epsilon_{i}(t),
$$

em que p é a ordem autorregressiva e q a ordem de médias móveis, $\lambda_{k}$ a ordem máxima de vizinhança do k-ésimo termo autorregressivo e $m_{k}$ a ordem máxima espacial do késimo termo de médias móveis, $L^{(l)}$ o operador da defasagem espacial definida na seção 3.1, e $\varepsilon_{i}(t)$ são os erros aleatórios que seguem uma distribuição normal com esperança $E\left(\varepsilon_{i}(t)\right)=0, \mathrm{e}$

$$
E\left[\epsilon_{i}(t) \epsilon_{j}(t+s)^{\prime}\right]=\left\{\begin{aligned}
\sigma^{2}, & \mathrm{i}=\mathrm{j}, \quad \mathrm{s}=0, \\
0, & \text { c.c. }
\end{aligned}\right.
$$

Escrevemos o modelo STARMA $\left(p_{\lambda_{1}, \ldots, \lambda_{p}}, q_{m_{1}, \ldots, m_{q}}\right)$ na forma matricial:

$$
\mathbf{Z}(t)=\sum_{k=1}^{p} \sum_{l=0}^{\lambda_{k}} \phi_{k l} \mathbf{W}^{l} \mathbf{Z}(t-k)-\sum_{k=1}^{q} \sum_{l=0}^{m_{k}} \theta_{k l} \mathbf{W}^{l} \boldsymbol{\epsilon}(t-k)+\boldsymbol{\epsilon}(t)
$$

Semelhante à classe do modelo ARMA, temos duas subclasses especiais para o modelo STARMA, que são os modelos STAR e STMA. No caso de permanencer somente a parte auto-regressiva, temos o modelo espaço-temporal autorregressivo $\operatorname{STAR}\left(p_{\lambda_{0}, \lambda_{1}, \ldots, \lambda_{p}}\right)$ é dado por:

$$
\mathbf{Z}(t)=\sum_{k=1}^{p} \sum_{l=0}^{\lambda_{k}} \phi_{k l} \mathbf{W}^{l} \mathbf{Z}(t-k)+\boldsymbol{\epsilon}(t)
$$

Quando o termo autorregressivo p é igual a zero, temos o modelo espaço-temporal média móvel $\operatorname{STMA}\left(q_{m_{1}, \ldots, m_{p}}\right)$ representado pela equação 3.6:

$$
\mathbf{Z}(t)=\boldsymbol{\epsilon}(t)-\sum_{k=1}^{q} \sum_{l=0}^{m_{k}} \theta_{k l} \mathbf{W}^{l} \boldsymbol{\epsilon}(t-k) .
$$

O modelo STARMA representa processos estacionários, isto é, sua média e a estrutura de covariância de $\mathbf{Z}(t)$ não se alteram com o tempo. Para que estas condições (condições de estacionariedade) serem satisfeitas, segundo Biz (2014), é necessário que todos os possíveis valores de $x_{u}$ que satisfaçam a equação

$$
\operatorname{det}\left[x_{u}^{p} I-\sum_{k=1}^{p} \sum_{l=0}^{\lambda_{k}} \phi_{k l} \mathbf{W}^{(l)} x_{u}^{p-k}\right]=0
$$

estejam dentro do círculo unitário $\left(\left|x_{u}\right|<1\right)$, isto é equilavente a determinar uma região de possíveis valores de $\phi_{k l}$ que resulta em um processo estacionário e com solução única. 
Agora para que $\mathbf{Z}(\mathrm{t})$ seja expressa como combinação linear ponderada de observações passadas e com seus pesos que convergem para zero, então é preciso que todos os possíveis valores de $x_{u}$ que satisfazem a seguinte equação

$$
\operatorname{det}\left[x_{u}^{q} I-\sum_{k=1}^{q} \sum_{l=0}^{m_{k}} \theta_{k l} \mathbf{W}^{(l)} x_{u}^{q-k}\right]=0
$$

e estejam dentro do círculo unitário $\left(\left|x_{u}\right|<1\right)$, assim o modelo é invertível.

Observam-se todos os modelos STAR são invertíveis e todos os modelos STMA são estacionários. (Pfeifer e Deutsch (1980a)).

Como a classe de modelos ARIMA, para o modelo STARMA, quando a propriedade de estacionariedade não for satisfeita por ter uma tendência, neste caso basta realizar d vezes de diferença necessária nas séries para torná-las estacionárias. A incorporação da diferençiação nas séries ao modelo STARMA gera o modelo espaço-temporal autorregressivo integrado de média móvel STARIMA, apresentado por Pfeifer e Deutsch (1980c).

$$
\triangle^{d} \mathbf{Z}(t)=\sum_{k=1}^{p} \sum_{l=0}^{\lambda_{k}} \phi_{k l} \mathbf{W}^{l} \mathbf{Z}(t-k)-\sum_{k=1}^{q} \sum_{l=0}^{m_{k}} \theta_{k l} \mathbf{W}^{l} \boldsymbol{\epsilon}(t-k)+\boldsymbol{\epsilon}(t) .
$$

Para Pfeifer e Deutsch (1981c) e Martins (2013), de forma análoga a classe de modelos SARIMA, na classe de modelos STARIMA também pode-se incorporar a componente sazonal, assim temos o modelo STARIMA Sazonal:

$$
\Phi_{P, \Lambda}\left(B^{s}\right) \phi_{p, \lambda}(B) \triangle_{s}^{D} \triangle^{d} \mathbf{Z}(t)=\Theta_{Q, M}\left(B^{s}\right) \theta_{q, m}(B) \boldsymbol{\epsilon}(t)
$$

em que

$\triangle^{d}=(1-B)^{d}$ é o operador diferença, d o número de diferenças;

$\triangle_{s}^{D}=\left(1-B^{s}\right)^{D}$ é o operador diferença sazonal s, D o número de diferenças sazonais;

$\phi_{p, \lambda}(B)=\mathbf{I}-\sum_{k=1}^{p} \sum_{l=0}^{\lambda_{k}} \phi_{k l} \mathbf{W}^{(l)} B^{k}$ é o operador autorregressivo espacial estacionário de ordem p;

$\Phi_{P, \Lambda}\left(B^{s}\right)=\mathbf{I}-\sum_{k=1}^{P} \sum_{l=0}^{\Lambda_{k}} \Phi_{k l} \mathbf{W}^{(l)} B^{s k}$ é o operador autorregressivo sazonal espacial estacionário de ordem $\mathrm{P}$;

$\theta_{q, m}(B)=\mathbf{I}-\sum_{k=1}^{q} \sum_{l=0}^{m_{k}} \theta_{k l} \mathbf{W}^{(l)} B^{k}$ é o operador de médias móveis espacial invertível de ordem q;

$\Theta_{Q, M}\left(B^{s}\right)=\mathbf{I}-\sum_{k=1}^{Q} \sum_{l=0}^{M_{k}} \Theta_{k l} \mathbf{W}^{(l)} B^{s k}$ é o operador de médias móveis espacial sazonal invertível de ordem $Q$. 


\subsection{Identificação}

Para os modelos univariados de Box e Jenkins no capítulo 2, apresentamos a função de autocovariância e a função de autocovariância parcial, que são essenciais para identificar a ordem q do modelo MA e a ordem p do modelo AR. Para a classe do modelo STARMA temos a função de autocovariância espaço-temporal, a covariância entre pontos defasados tanto no tempo quanto no espaço combinando a covariância entre todos os pares possíveis de locais.

Segundo Biz (2014) e Pfeifer e Deutsch (1980a), a covariância espaço-temporal (a covariância média ponderada) entre vizinhos da l-ésima ordem e k-ésima ordem no tempo de defasagem s é definida como:

$$
\gamma_{l k}(s)=E\left\{\sum_{i=1}^{N} \frac{L^{(l)} Z_{i}(t) L^{(k)} Z_{i}(t+s)}{N}\right\} .
$$

E a forma matricial da função de covariância espaço-temporal é

$$
\gamma_{l k}(s)=E\left\{\frac{\left[\mathbf{W}^{(l)} \mathbf{Z}(t)\right]^{\prime}\left[\mathbf{W}^{(k)} \mathbf{Z}(t+s)\right]}{N}\right\} .
$$

Pela propriedade de $E\left[\mathbf{X}^{\prime} \mathbf{A X}\right]=\operatorname{tr}\left[\mathbf{A} E\left[\mathbf{X X}^{\prime}\right]\right]$, podemos escrever a equação 3.18 como

$$
\gamma_{l k}(s)=\operatorname{tr}\left\{\frac{\mathbf{W}^{(k)^{\prime}} \mathbf{W}^{(l)} \Gamma(s)}{N}\right\},
$$

em que tr é o traço de uma matriz, e $\boldsymbol{\Gamma}(s)=E\left[\mathbf{Z}(t) \mathbf{Z}(t+s)^{\prime}\right]$. Sendo $\hat{\boldsymbol{\Gamma}}(s)$ o estimador para $\Gamma(s)$ :

$$
\hat{\boldsymbol{\Gamma}}(s)=\sum_{t=1}^{T-s} \frac{\mathbf{z}(t) \mathbf{z}(t+s)^{\prime}}{T-s} .
$$

z são as observações das variáveis $\mathbf{Z}$, substituindo a equação 3.20 em 3.19, obteremos o estimador da função de covariância espaço-temporal $\hat{\gamma}_{l k}(s)$,

$$
\hat{\gamma}_{l k}(s)=\frac{1}{N} \operatorname{tr}\left\{\mathbf{W}^{(k)^{\prime}} \mathbf{W}^{(l)} \sum_{t=1}^{T-s} \frac{\mathbf{z}(t) \mathbf{z}(t+s)^{\prime}}{T-s}\right\} .
$$

Quando $k=0$ e $l=0$, temos

$$
\gamma_{00}(s)=\frac{1}{N} \operatorname{tr}\{\boldsymbol{\Gamma}(s)\}
$$

que é a autocovariância média da s-ésima defasagem para todos os $\mathrm{N}$ locais. 
E se $k=1$ e $l=0$

$$
\gamma_{10}(s)=\frac{1}{N} \operatorname{tr}\left\{\mathbf{W}^{(1)} \boldsymbol{\Gamma}(s)\right\}
$$

que é a covariância média de todos os locais e suas respectivas vizinhanças de ordem 1 com defasagem de tempo s.

Segundo Pfeifer e Deutsch (1980a), uma propriedade importante da covariância espaço-temporal é

$$
\gamma_{l k}(s)=\gamma_{k l}(-s)
$$

pela propriedade de matriz $\operatorname{tr}[X Y]=\operatorname{tr}[Y X]$ e $\boldsymbol{\Gamma}(s)=\boldsymbol{\Gamma}(-s)^{\prime}$.

A função de autocorrelação espaço-temporal STFAC de acordo com Martin e Oeppen (1975) não é simples como é na classe de modelos univariados de Box-Jenkins. Antes de definir a função autocorrelação espaço-temporal, é necessária a condição da variância constante para todas as defasagens espaciais. Assim, sendo a definição da função de autocorrelação espaço-temporal entre l-ésima e k-ésima ordem de vizinhança na defasagem temporal s é

$$
\rho_{l k}(s)=\frac{\gamma_{l k}(s)}{\sqrt{\left[\gamma_{l l}(0) \gamma_{k k}(0)\right]}}
$$

Dado que a estimativa amostral da variância se mostra relativamente constante para todas as defasagens espaciais (Pfeifer e Deutsch (1980a)), as estimativas amostrais da autocorrelação espaço-temporal são dadas por

$$
\hat{\rho}_{l k}(s)=\frac{\hat{\gamma}_{l k}(s)}{\sqrt{\left[\hat{\gamma}_{l l}(0) \hat{\gamma}_{k k}(0)\right]}}=\frac{\sum_{i=1}^{N} \sum_{t=1}^{T-s} L^{(l)} z_{i}(t) L^{(k)} z_{i}(t+s)}{\sqrt{\sum_{i=1}^{N} \sum_{t=1}^{T}\left(L^{(l)} z_{i}(t)\right)^{2} \sum_{i=1}^{N} \sum_{t=1}^{T}\left(L^{(k)} z_{i}(t)\right)^{2}}} .
$$

Quando $k=0$ temos a autocorrelação espaço-temporal entre os locais e seus respectivos vizinhos de ordem l, e escrevemos na forma matricial segundo Biz (2014):

$$
\hat{\rho}_{l 0}(s)=\frac{T}{T-s} \frac{\left.\sum_{t=1}^{T-s}\left[\mathbf{W}_{l} \mathbf{z}(t)\right]^{\prime} \mathbf{z}(t+s)\right]}{\sqrt{\sum_{t=1}^{T}\left[\mathbf{W}_{l} \mathbf{z}(t)\right]^{\prime}\left[\mathbf{W}_{l} \mathbf{z}(t)\right] \sum_{t=1}^{T} \mathbf{z}^{\prime}(t) \mathbf{z}(t)}} .
$$

Segundo Martin e Oeppen (1975), o sistema de cálculo da função de autocorrelação parcial espaço-temporal STFACP é análogo aos modelos univariados de Box-Jenkins, que pode ser verificada através das equações de Yule-Walker. Multiplicando ambos os lados do modelo $\operatorname{STAR}\left(k_{0,1, \ldots, \lambda}\right)$ por $\left[\mathbf{W}^{(h)} \mathbf{Z}(t-s)\right]^{\prime}$, temos

$$
\mathbf{Z}(t-s)^{\prime} \mathbf{W}^{(h)^{\prime}} \mathbf{Z}(t)=\sum_{j=1}^{k} \sum_{l=0}^{\lambda} \phi_{j l} \mathbf{Z}(t-s)^{\prime} \mathbf{W}^{(h)^{\prime}} \mathbf{W}^{(l)} \mathbf{Z}(t-j)+\mathbf{Z}(t-s)^{\prime} \mathbf{W}^{(h)^{\prime}} \boldsymbol{\epsilon}(t)
$$


Aplicando a esperança e dividindo ambos lados por N, tem-se

$$
\gamma_{h 0}(s)=\sum_{j=1}^{k} \sum_{l=0}^{\lambda} \phi_{j l} \gamma_{h l}(s-j),
$$

uma vez que $E\left[\mathbf{z}(t-j)^{\prime} \boldsymbol{\epsilon}(t)\right]=0$, para $s>0$. Assim, para $s=0,1,2, \ldots, k$ e $h=$ $0,1,2, \ldots, \lambda$, segundo Martin e Oeppen (1975) temos o sistema de equações apresentado na Figura 3.2.

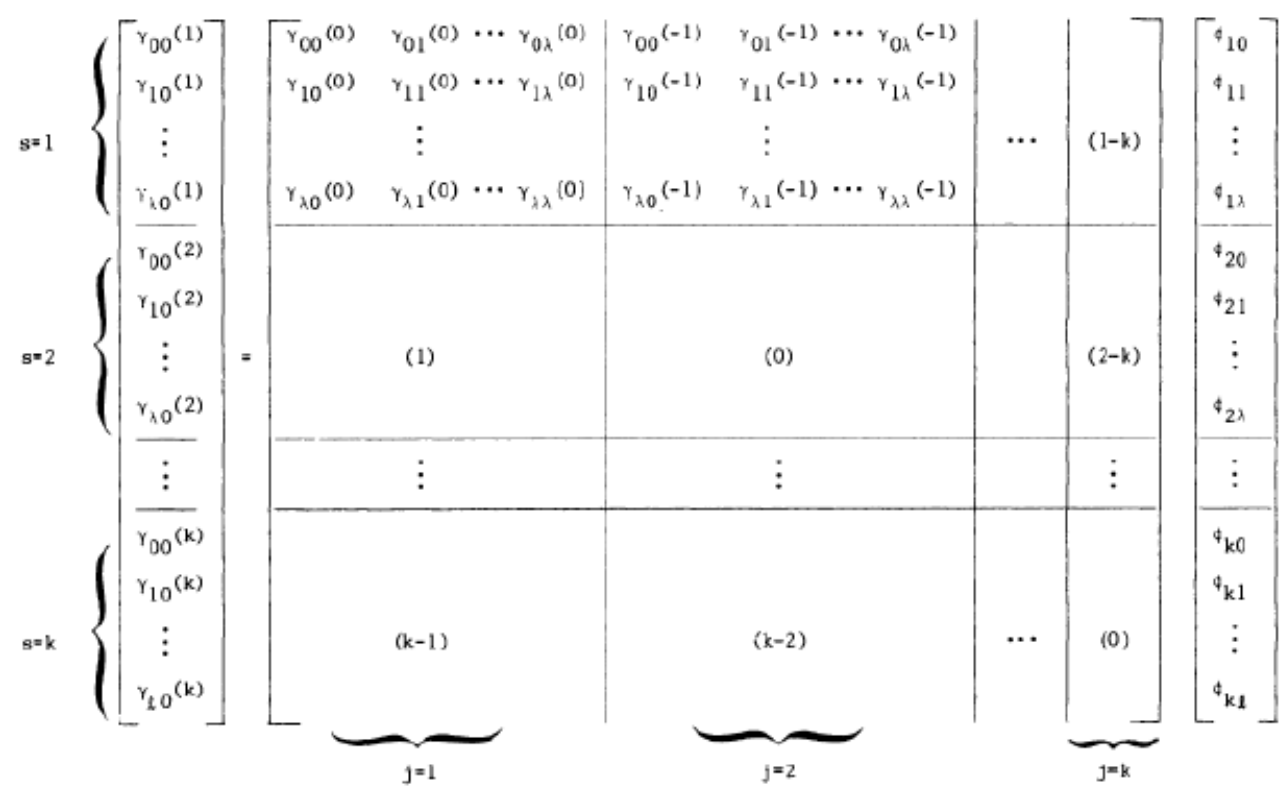

Figura 3.2: Sistema de equações Yule-Walker, Pfeifer e Deutsch (1980b)

Segunda Pfeifer e Deutsch (1980b) e Martins (2013), o último coeficiente $\phi_{k l}^{\prime}$ obtido resolvendo as equações do sistema Figura $3.2 \mathrm{com} l=0,1,2, \ldots, \lambda$ e $k=1,2, \ldots$, é chamado de função de autocorrelação parcial espaço-temporal de ordem espacial $\lambda$. A escolha do $\lambda$ deve ser tão grande quanto a ordem máxima espacial por hipótese de qualquer modelo, porém pelo Pfeifer e Deutsch (1980b), para $\lambda$ maior como 3 ou 4, obtém-se grandes sistemas, portanto, $\lambda=2$ é suficiente. Para calcular os $\gamma_{h l}(s)$ da equação 3.21 basta substituir por seus estimativas $\hat{\gamma}_{h l}(s)$ sucessivamente para $l=0,1,2, \ldots, \lambda$ e $k=1,2, \ldots$,

No capitulo 2, vemos que nos modelos autorregressivos univariados de ordem $\mathrm{p}$ $\mathrm{AR}(\mathrm{p})$, a função de autocorrelação decai exponencialmente e a função de autocorrelação parcial é igual a zero após lag p. Agora, os processos de $\operatorname{STAR}\left(p_{\lambda_{0}, \lambda_{1}, \ldots, \lambda_{p}}\right)$ possuem uma função de autocorrelação espaço-temporal que diminuem gradativamente tanto para o espaço quanto para o tempo e a função de autocorrelação parcial espaço-temporal é igual a zero após o lag p no tempo e lag $\lambda_{p}$ no espaço. Para os modelos univariados de médias móveis de ordem q MA(q), temos a função de autocorrelação igual a zero após lag q, e a função de autocorrelação parcial decai exponencialmente ao longo do tempo, já o 
modelo de $\operatorname{SMA}\left(q_{m_{0}, m_{1}, \ldots, m_{q}}\right)$ tem uma função de autocorrelação espaço-temporal que corta após o lag temporal q e o lag espacial $m_{q}$, e a função de autocorrelação parcial espaçotemporal que decai gradativamente tanto para o tempo quanto para o espaço. E para o modelo STARMA, temos STPAC e STFACP decaem gradativamente, temporalmente e espacialmente.

Tabela 3.1: Comportamento das STFAC e STFACP da classe de modelos STARMA

\begin{tabular}{ccc}
\hline Modelos & STFAC & STFACP \\
\hline $\operatorname{STAR}\left(p_{\lambda_{1}, \ldots, \lambda_{p}}\right)$ & decai gradativamente & $\phi_{k l}=0, k>p$ e $l>\lambda_{p}$ \\
$\operatorname{SMA}\left(q_{m_{1}, \ldots, m_{q}}\right)$ & $\rho_{l 0}(s)=0, s>q$ e $l>m_{q}$ & decai gradativamente \\
$\operatorname{STARMA}\left(p_{\lambda_{1}, \ldots, \lambda_{p}}, q_{m_{1}, \ldots, m_{q}}\right)$ & decai gradativamente & decai gradativamente \\
\hline
\end{tabular}

Para selecionar o melhor modelo STARMA, segundo Pfeifer e Deutsch (1980a), é considerado o princípio da parcimônia, isto é escolher o modelo com menor número de parâmetros para estimação, e o critério de informação BIC,

$$
B I C=N T \ln \left(\hat{\sigma}^{2}\right)+2 b \ln (T),
$$

em que $\hat{\sigma}^{2}$ é a estimativa da variância residual, b a quantidade de parâmetros, $\mathrm{N}$ o número de regiões e $\mathrm{T}$ o tamanho das séries.

\subsection{Estimação}

\subsubsection{Método de Máxima Verossimilhança}

O método de estimação para os modelos da classe STARMA, segundo Pfeifer e Deutsch (1980a), é a máxima verossimilhança. Temos $\boldsymbol{\epsilon}=\left[\epsilon_{1}(1), \ldots, \epsilon_{1}(T), \ldots, \epsilon_{N}(1), \ldots \epsilon_{N}(T)\right]^{\prime}$, com $\mathrm{N}$ e $\mathrm{T}$, o número total de região e de observação, supondo que $\boldsymbol{\epsilon}$ possui uma distribuição normal multivariada com a média zero e a matriz de covariância $\sigma_{\epsilon}^{2} \mathbf{I}_{N T}$. Os parâmetros que precisam ser estimados são $\boldsymbol{\Phi}=\left[\phi_{10}, \phi_{11}, \ldots, \phi_{1 \lambda_{1}}, \ldots, \phi_{p 0}, \ldots, \phi_{p \lambda_{p}}\right]^{\prime}$, $\Theta=\left[\theta_{10}, \theta_{11}, \ldots, \theta_{1 m_{1}}, \ldots, \theta_{q 0}, \ldots, \theta_{q m_{q}}\right]^{\prime}$, e $\sigma_{\epsilon}^{2}$. A função densidade de probabilidade conjunta condicional é escrita como

$$
\begin{aligned}
f\left(\boldsymbol{\epsilon} \mid \boldsymbol{\Phi}, \boldsymbol{\Theta}, \sigma^{2}\right) & =(2 \pi)^{-\frac{T N}{2}}\left|\sigma^{2} \boldsymbol{I}_{N T}\right|^{-\frac{1}{2}} \exp \left\{-\frac{1}{2 \sigma^{2}} \boldsymbol{\epsilon}^{\prime} \boldsymbol{I} \boldsymbol{\epsilon}\right\} \\
& =(2 \pi)^{-\frac{T N}{2}} \sigma^{2^{-\frac{T N}{2}}} \exp \left\{-\frac{S_{*}(\boldsymbol{\Phi}, \boldsymbol{\Theta})}{2 \sigma^{2}}\right\}
\end{aligned}
$$

em que $S_{*}(\boldsymbol{\Phi}, \boldsymbol{\Theta})=\boldsymbol{\epsilon}^{\prime} \boldsymbol{I} \boldsymbol{\epsilon}=\sum_{i=1}^{N} \sum_{t=1}^{T} \epsilon_{i}^{2}(t)$.

Pelo Pfeifer e Deutsch (1980a), é preciso encontrar as estimativas dos parâmetros que maximizam a função de verossimilhança condicional, isto é obter os valores de $\boldsymbol{\Phi}$ e 
$\Theta$ que minimizam $S_{*}(\boldsymbol{\Phi}, \boldsymbol{\Theta})$, com $\boldsymbol{\epsilon}(t)$ erros aleatórios não observáveis calculados a partir de observações de $\boldsymbol{Z}(t)$ recursivamente usando a equação:

$$
\boldsymbol{\epsilon}(t)=\mathbf{Z}(t)-\sum_{k=1}^{p} \sum_{l=0}^{\lambda_{k}} \phi_{k l} \mathbf{W}^{l} \mathbf{Z}(t-k)-\sum_{k=1}^{q} \sum_{l=0}^{m_{k}} \theta_{k l} \mathbf{W}^{l} \boldsymbol{\epsilon}(t-k)
$$

e com os valores iniciais $\boldsymbol{Z}(t)$ e $\boldsymbol{\epsilon}(t)$ iguais a zero para $t<1$.

Aplicamos o logaritmo neperiano na função de densidade conjunta condicional, temos

$$
l_{*}\left(\boldsymbol{\Phi}, \boldsymbol{\theta}, \sigma^{2}\right)=-\frac{T N}{2} \ln (2 \pi)-\frac{T N}{2} \sigma^{2}-\frac{S_{*}(\boldsymbol{\Phi}, \boldsymbol{\Theta})}{2 \sigma^{2}} .
$$

Então $\hat{\boldsymbol{\Phi}}, \hat{\boldsymbol{\Theta}}$ e $\hat{\sigma}^{2}$ são os estimadores de máxima verossimilhança condicional de $\sigma^{2}, \boldsymbol{\Phi}, \boldsymbol{\Theta}$ que minimizam $S_{*}(\boldsymbol{\Phi}, \boldsymbol{\Theta})$ e $\hat{\sigma}^{2}=\frac{S_{*}(\hat{\boldsymbol{\Phi}}, \hat{\boldsymbol{\Theta}})}{T N}$.

Para Biz (2014) esse procedimento é também equivalente a encontrar os valores de $\hat{\boldsymbol{\Phi}}, \hat{\boldsymbol{\Theta}}$ que minimizam $\operatorname{tr}(\boldsymbol{M})$, em que $\boldsymbol{M}=\frac{\sum_{t=1}^{T} \hat{\boldsymbol{\epsilon}}(t) \hat{\boldsymbol{\epsilon}}^{\prime}(t)}{T}$.

O modelo STAR possui a forma linear, isto é, poder ser escrito na forma de regressão linear $\boldsymbol{Y}=\boldsymbol{X} \boldsymbol{\beta}+\boldsymbol{\epsilon}$, e com isso o vetor de parâmetros $\boldsymbol{\Phi}$ pode ser estimado pelo método de mínimos quadrados,

$$
\left(\boldsymbol{X}^{\prime} \boldsymbol{X} \hat{\boldsymbol{\Phi}}\right)=\boldsymbol{X}^{\prime} \boldsymbol{Z}
$$

Portanto, os intervalos de confianças para as estimativas dos parâmetros do modelo STAR podem ser construídos usando o resultado da teoria de regressão linear, desde que

$$
\frac{(\boldsymbol{\Phi}-\hat{\mathbf{\Phi}})^{\prime} \boldsymbol{X}^{\prime} \boldsymbol{X}(\boldsymbol{\Phi}-\hat{\mathbf{\Phi}})}{\frac{C S_{*}(\hat{\mathbf{\Phi}})}{T N-C}} \sim F_{(C, T N-C)},
$$

em que $S_{*}(\hat{\boldsymbol{\Phi}})$ é a soma de quadrado residual e C o número dos parâmetros do modelo. Note que pela natureza de séries temporais, a suposição de independência da regressão linear não é consolidada. Para Pfeifer e Deutsch (1980a) e Biz (2014), utiliza-se o resultado da regressão linear, o sistema Yule-Walker para o modelo STAR, pois essa estimativa é aproximada.

Agora para o modelo STMA e STARMA que não possuem a natureza de linear, a determinação da região de confiança dos parâmetros não é fácil. Isso é porque a soma de quadrados $S_{*}(\Phi, \Theta)$ e sua função de verossimilhança não são simétricas, o que não resulta em uma expressão algébrica fechada definida. Neste caso, deve-se usar a otimização não linear. De acordo com Pfeifer e Deutsch (1980a), para resolver o problema de não simetria, é possível usar uma função de verossimilhança aproximada, que parte de uma expansão de Taylor da soma de quadrados $S_{*}(\boldsymbol{\Phi}, \boldsymbol{\Theta})$ sobre as estimativas de mínimos quadrados.

A soma de quadrados $S_{*}(\boldsymbol{\Phi}, \boldsymbol{\Theta})$ pode ser aproximada por:

$$
S_{*}(\boldsymbol{\Phi}, \boldsymbol{\Theta})=S(\boldsymbol{\zeta}) \approx S(\hat{\boldsymbol{\zeta}})+(\boldsymbol{\zeta}-\hat{\boldsymbol{\zeta}})^{\prime} \boldsymbol{Q}(\boldsymbol{\zeta}-\hat{\boldsymbol{\zeta}})
$$


em que $\boldsymbol{\zeta}^{\prime}=\left(\boldsymbol{\Phi}^{\prime}, \boldsymbol{\Theta}^{\prime}\right)$, e $\boldsymbol{Q}=\frac{1}{2}\left[\frac{\partial^{2} S(\boldsymbol{\zeta})}{\partial \zeta_{i} \partial \zeta_{j}}\right]_{\hat{\boldsymbol{\zeta}}}$, com i=1,2,.., C, C a quantidade total de parâmetros.

Uma vez que $S_{*}(\boldsymbol{\Phi}, \boldsymbol{\Theta})=\sum_{t=1}^{T} \boldsymbol{\epsilon}(t) \boldsymbol{\epsilon}(t)^{\prime}$, temos

$$
\frac{\partial S(\boldsymbol{\zeta})}{\partial \zeta_{i}}=\left.\sum_{t=1}^{T} 2 \boldsymbol{\epsilon}(t)^{\prime} \frac{\partial \boldsymbol{\epsilon}(t)}{\partial \zeta_{i}}\right|_{\hat{\boldsymbol{\zeta}}}=0
$$

e

$$
\frac{1}{2}\left[\frac{\partial^{2} S(\boldsymbol{\zeta})}{\partial \zeta_{i} \partial \zeta_{j}}\right]_{\hat{\boldsymbol{\zeta}}}=\left.\sum_{t=1}^{T} \boldsymbol{\epsilon}(t)^{\prime} \frac{\partial^{2} \boldsymbol{\epsilon}(t)}{\partial \zeta_{i} \partial \zeta_{j}}\right|_{\hat{\boldsymbol{\zeta}}}+\left.\sum_{t=1}^{T} \frac{\partial \boldsymbol{\epsilon}(t)^{\prime}}{\partial \zeta_{i}} \frac{\partial \boldsymbol{\epsilon}(t)}{\partial \zeta_{j}}\right|_{\hat{\boldsymbol{\zeta}}}
$$

Segundo $\operatorname{Biz}(2014)$, como $\left.\frac{\partial^{2} \boldsymbol{\epsilon}(t)}{\partial \zeta_{i} \partial \zeta_{j}}\right|_{\hat{\zeta}}$ é uma função de $\boldsymbol{\epsilon}$ ocorridos antes do tempo T e espera-se que $\mathrm{E}\left[\boldsymbol{\epsilon}(t) \boldsymbol{\epsilon}(t-C)^{\prime}\right]=0$, para $\mathrm{C} \geqslant 1$, então a matriz $\boldsymbol{Q}$ pode ser escrita como $\boldsymbol{Q}=\boldsymbol{A}^{\prime} \boldsymbol{A}$, em que

$$
\mathbf{A}=\left[\begin{array}{cccc}
\left.\frac{\partial \boldsymbol{\epsilon}(1)}{\partial \zeta_{1}}\right|_{\hat{\boldsymbol{\zeta}}} & \left.\frac{\partial \boldsymbol{\epsilon}(1)}{\partial \zeta_{2}}\right|_{\hat{\boldsymbol{\zeta}}} & \cdots & \left.\frac{\partial \boldsymbol{\epsilon}(1)}{\partial \zeta_{C}}\right|_{\hat{\boldsymbol{\zeta}}} \\
\left.\frac{\partial \boldsymbol{\epsilon}(2)}{\partial \zeta_{1}}\right|_{\hat{\boldsymbol{\zeta}}} & \left.\frac{\partial \boldsymbol{\epsilon}(2)}{\partial \zeta_{2}}\right|_{\hat{\boldsymbol{\zeta}}} & \cdots & \left.\frac{\partial \boldsymbol{\epsilon}(2)}{\partial \zeta_{C}}\right|_{\hat{\boldsymbol{\zeta}}} \\
\vdots & \vdots & \ddots & \vdots \\
\left.\frac{\partial \boldsymbol{\epsilon}(T)}{\partial \zeta_{1}}\right|_{\hat{\boldsymbol{\zeta}}} & \left.\frac{\partial \boldsymbol{\epsilon}(T)}{\partial \zeta_{2}}\right|_{\hat{\boldsymbol{\zeta}}} & \cdots & \left.\frac{\partial \boldsymbol{\epsilon}(T)}{\partial \zeta_{C}}\right|_{\hat{\boldsymbol{\zeta}}}
\end{array}\right]
$$

Por isso a soma dos quadrados $S_{*}(\boldsymbol{\Phi}, \boldsymbol{\Theta})$ é aproximada por:

$$
S_{*}(\boldsymbol{\Phi}, \boldsymbol{\Theta}) \approx S(\hat{\boldsymbol{\zeta}})+(\boldsymbol{\zeta}-\hat{\boldsymbol{\zeta}})^{\prime} \boldsymbol{Q}(\boldsymbol{\zeta}-\hat{\boldsymbol{\zeta}})
$$

A região de confiança para $(\boldsymbol{\Phi}, \boldsymbol{\Theta})^{\prime}$ pode ser obtida segundo Pfeifer e Deutsch (1980a):

$$
S(\boldsymbol{\zeta})=S(\hat{\boldsymbol{\zeta}})+\frac{b}{T N-b} S(\hat{\boldsymbol{\zeta}}) F_{(b, T N-b, \alpha)}
$$

em que $\alpha$ é o nível de significância.

Testamos se um particular parâmetro $\zeta_{h}$ é zero, $\mathrm{h}=1,2, \ldots, \mathrm{C}$, temos a estatística

$$
\frac{(T N-K)\left[S\left(\hat{\boldsymbol{\zeta}}^{*}\right)-S(\hat{\boldsymbol{\zeta}})\right]}{S(\hat{\boldsymbol{\zeta}})} \sim F_{(1, T N-C, \alpha)},
$$

com C o número de parâmetros, $\hat{\boldsymbol{\zeta}}$ a estimativa de mínimos quadrados do vetor de parâmteros completo e $\hat{\boldsymbol{\zeta}}^{*}$ a estimativa de mínimos quadrados com $\zeta_{h}=0$.

Conforme Rao e Antunes (2004) e Biz (2014), a matriz $\boldsymbol{Q}$ deve ser estimada numericamente e $S(\boldsymbol{\zeta})$ deve ser substituida pela soma de quadrados condicional $S_{*}(\boldsymbol{\zeta})$ quando a função de verossimilhança é usada. 
O intervalo de confiança para $\sigma^{2}$ é calculado por

$$
\frac{S_{*}(\boldsymbol{\zeta})}{\left(\sigma^{2} \mid \boldsymbol{z}(1), \boldsymbol{z}(2), \ldots, \boldsymbol{z}(T)\right)} \sim \chi_{T N-C}^{2}
$$

\subsubsection{Estimação por Filtro de Kalman}

O modelo STARMA foi desenvolvido nas décadas de oitenta e passou por um tempo de esquecimento por causa de dificuldade de estimação. Nos últimos anos, começaram a surgir implementações computacionais do modelo, por exemplo, o pacote Starma do software R é um deles utilizando o algorítimo de iteração de estimação Filtro de Kalman baseado no Cipra e Motykova (1987), o Filtro de Kalman para modelos multivariados VARMA.

A filtragem do Kalman pode ser utilizada como um instrumento muito prático para a estimação adaptativa não só em aplicações técnicas, mas também para séries temporais mais curtas. No que diz respeito à filtragem kalman na análise de séries temporais, alguns autores preferem construir, por meio do Filtro de Kalman, a função de verossimilhança exata dos modelos de séries temporais. Entretanto, para Cipra e Motykova (1987), o Filtro de Kalman fornece diretamente as estimativas recursivas que são ótimas no sentido do princípio dos mínimos quadrados.

\subsubsection{Introdução}

Aplicamos o Filtro de Kalman no modelo espaço de estados, um sistema linear dinâmico discreto:

$$
\begin{gathered}
\mathbf{x}_{t+1}=\boldsymbol{\Phi}_{t} \mathbf{x}_{t}+\Gamma_{t} \mathbf{w}_{t+1} \\
\mathbf{y}_{t}=\mathbf{M}_{t} \mathbf{x}_{t}+\mathbf{v}_{t}
\end{gathered}
$$

em que 3.33 é a equação de estado e 3.34 é a equação de observação do sistema. $\mathbf{x}_{t}$ é o vetor de variável estado de dimensão $\left(m_{t}, 1\right)$ no tempo t; $\mathbf{y}_{t}$ é o vetor de observação de dimensão $\left(n_{t}, 1\right)$ no t; $\boldsymbol{\Phi}_{t}, \boldsymbol{\Gamma}_{t}, \mathbf{M}_{t}$ são matrizes do tipo $\left(m_{t+1}, m_{t}\right),\left(m_{t+1}, q_{t+1}\right),\left(n_{t}, m_{t}\right)$, respectivamente $\left(\boldsymbol{\Phi}_{t}\right.$ é a matriz de estado de transição ou matriz de sistema, $\boldsymbol{\Gamma}_{t}$ é a matriz de entrada e $\mathbf{M}_{t}$ é a matriz de observação no tempo t); $\mathbf{w}_{t}$ e $\mathbf{v}_{t}$ são vetores aleatórios de dimensão $\left(q_{t}, 1\right)$ e $\left(n_{t}, 1\right)$ cumprindo as condições

$$
\begin{aligned}
& \mathrm{E}\left(\mathbf{w}_{t}\right)=0, \mathrm{E}\left(\mathbf{v}_{t}\right)=0, \\
& \operatorname{Var}\left(\mathbf{w}_{t}\right)=\mathbf{Q}_{t}, \operatorname{Var}\left(\mathbf{v}_{t}\right)=\mathbf{R}_{t}, \\
& \operatorname{Cov}\left(\mathbf{w}_{s}, \mathbf{w}_{t}\right)=0, \operatorname{Cov}\left(\mathbf{v}_{s}, \mathbf{v}_{t}\right)=0, s \neq t \\
& \operatorname{Cov}\left(\mathbf{w}_{s}, \mathbf{v}_{t}\right)=0,
\end{aligned}
$$


com matrizes de variância $\mathbf{Q}_{t}$ e $\mathbf{R}_{t}$ de dimensão $\left(q_{t}, q_{t}\right)$ e $\left(n_{t}, n_{t}\right)$ no tempo t. Além disso, o valor inicial $\mathbf{x}_{0}$ da variável de estado é assumido para cumprir

$$
\operatorname{Cov}\left(\mathbf{x}_{0}, \mathbf{w}_{t}\right)=0, \operatorname{Cov}\left(\mathbf{x}_{0}, \mathbf{v}_{t}\right)=0
$$

Se $\mathbf{Y}_{t}$ denota o espaço $\left(n_{1}+\ldots+n_{t}\right)$ dimensional de Hilbert gerado pelas componentes dos vetores aleatórios $\mathbf{y}_{1}, \ldots, \mathbf{y}_{t}$, então o filtro de Kalman produz recursivamente as projeções ortogonais $\hat{\mathbf{x}}_{t}^{t}$ e $\hat{\mathbf{x}}_{t+1}^{t}$ de $\mathbf{x}_{t}$ e $\mathbf{x}_{t+1}$ em $\mathbf{Y}_{t}$ juntos com as matrizes

$$
\mathbf{P}_{t}^{t}=\mathrm{E}\left(\mathbf{x}_{t}-\hat{\mathbf{x}}_{t}^{t}\right)\left(\mathbf{x}_{t}-\hat{\mathbf{x}}_{t}^{t}\right)^{\prime}, \mathbf{P}_{t+1}^{t}=\mathrm{E}\left(\mathbf{x}_{t+1}-\hat{\mathbf{x}}_{t+1}^{t}\right)\left(\mathbf{x}_{t+1}-\hat{\mathbf{x}}_{t+1}^{t}\right)^{\prime}
$$

O filtro pode ser escrito na forma

$$
\begin{gathered}
\hat{\mathbf{x}}_{t+1}^{t}=\boldsymbol{\Phi}_{t} \hat{\mathbf{x}}_{t}^{t}, \\
\mathbf{P}_{t+1}^{t}=\boldsymbol{\Phi}_{t} \mathbf{P}_{t}^{t} \boldsymbol{\Phi}_{t}^{\prime}+\boldsymbol{\Gamma}_{t} \mathbf{Q}_{t+1} \boldsymbol{\Gamma}_{t}^{\prime}, \\
\hat{\mathbf{x}}_{t}^{t}=\hat{\mathbf{x}}_{t}^{t-1}+\mathbf{K}_{t}\left(\mathbf{y}_{t}-\mathbf{M}_{t} \hat{\mathbf{x}}_{t}^{t-1}\right), \\
\mathbf{P}_{t}^{t}=\left(\mathbf{I}-\mathbf{K}_{t} \mathbf{M}_{t}\right) \mathbf{P}_{t}^{t-1},
\end{gathered}
$$

em que

$$
\mathbf{K}_{t}=\mathbf{P}_{t}^{t-1} \mathbf{M}_{t}^{\prime}\left(\mathbf{M}_{t} \mathbf{P}_{t}^{t-1} \mathbf{M}_{t}^{\prime}+\mathbf{R}_{t}\right)^{-1}=\mathbf{P}_{t}^{t} \mathbf{M}_{t}^{\prime} \mathbf{R}_{t}^{-1} .
$$

A matriz $\mathbf{M}_{t} \mathbf{P}_{t}^{t-1} \mathbf{M}_{t}^{\prime}+\mathbf{R}_{t}$ é suposta ser regular (isto é positivamente definida) na primeira expressão da equação 3.39 e a matriz $\mathbf{R}_{t}$ é suposta ser regular para $\mathbf{K}_{t}$ na segunda expressão da 3.39 (a regulariedade de $\mathbf{R}_{t}$ também garante a regulariedade da $\left.\mathbf{M}_{t} \mathbf{P}_{t}^{t-1} \mathbf{M}_{t}^{\prime}+\mathbf{R}_{t}\right)$.

As relações 3.35 e 3.36 são os passos de predição e 3.37 até 3.38 são as etapas de correção do algorítmo de filtragem de Kalman. Se conectar esses dois passos, obviamente, pode-se escrever

$$
\begin{gathered}
\hat{\mathbf{x}}_{t+1}=\boldsymbol{\Phi}_{t} \hat{\mathbf{x}}_{t}+\mathbf{K}_{t+1}\left(\mathbf{y}_{t+1}-\mathbf{M}_{t+1} \boldsymbol{\Phi}_{t} \hat{\mathbf{x}}_{t}\right), \\
\mathbf{P}_{t+1}=\left(\mathbf{I}-\mathbf{K}_{t+1} \mathbf{M}_{t+1}\right)\left(\boldsymbol{\Phi}_{t} \mathbf{P}_{t} \boldsymbol{\Phi}_{t}^{\prime}+\boldsymbol{\Gamma}_{t} \mathbf{Q}_{t+1} \boldsymbol{\Gamma}_{t}^{\prime}\right), \\
\mathbf{K}_{t+1}=\left(\boldsymbol{\Phi}_{t} \mathbf{P}_{t} \boldsymbol{\Phi}_{t}^{\prime}+\boldsymbol{\Gamma}_{t} \mathbf{Q}_{t+1} \boldsymbol{\Gamma}_{t}^{\prime}\right) \mathbf{M}_{t+1}^{\prime}\left[\mathbf{M}_{t+1}\left(\boldsymbol{\Phi}_{t} \mathbf{P}_{t} \boldsymbol{\Phi}_{t}^{\prime}+\boldsymbol{\Gamma}_{t} \mathbf{Q}_{t+1} \boldsymbol{\Gamma}_{t}^{\prime}\right) \mathbf{M}_{t+1}^{\prime}+\mathbf{R}_{t+1}\right]^{-1} \\
=\mathbf{P}_{t+1} \mathbf{M}_{t+1}^{\prime} \mathbf{R}_{t+1}^{-1},
\end{gathered}
$$

onde colocamos por simplicidade

$$
\hat{\mathbf{x}}_{t}=\hat{\mathbf{x}}_{t}^{t}, \mathbf{P}_{t}=\mathbf{P}_{t}^{t}
$$


Caso a matriz de sistema $\boldsymbol{\Phi}$ e a matriz de observação $\mathbf{M}$ sejam constantes em tempo, então a projeção ortogonal de $\hat{\mathbf{x}}_{t+k}^{t}$ de $\mathbf{x}_{t+k}$ em $\mathbf{Y}_{t}$ tem a forma

$$
\hat{\mathbf{x}}_{t+k}^{t}=\Phi^{k} \hat{\mathbf{x}}_{t}^{t}, k \geqslant 0,
$$

para que a predição $\hat{\mathbf{y}}_{t+k}^{t}$ de $\mathbf{y}_{t+k}$ no tempo t possa ser escrita como

$$
\hat{\mathbf{y}}_{t+k}^{t}=\mathbf{M} \hat{\mathbf{x}}_{t+k}^{t}=\mathbf{M} \Phi^{k} \hat{\mathbf{x}}_{t}^{t}, k \geqslant 0
$$

\subsubsection{Estimação de parâmetros adaptativos em modelos de séries temporais por Filtro de Kalman}

Primeiro, falaremos brevemente os resultados da estimação de parâmetros adaptativos no modelo de regressão linear clássica da forma

$$
y_{t}=\mathbf{z}_{t}^{\prime} \mathbf{b}+\epsilon_{t}
$$

em que $\mathbf{z}_{t}=\left(z_{1, t}, \ldots, z_{\gamma, t}\right)^{\prime}$ é o vetor de dimensão $(\gamma, 1)$ dos regressores no tempo $\mathrm{t}, \mathbf{b}=$ $\left(b_{1}, \ldots, b_{\gamma}\right)^{\prime}$ é o vetor de dimensão $(\gamma, 1)$ dos parâmetros de regressão e $\epsilon_{t}$ é o residual no tempo t de modo que $\mathrm{E}\left(\epsilon_{t}\right)=0, \operatorname{Var}\left(\epsilon_{t}\right)=\sigma^{2}, \operatorname{Cov}\left(\epsilon_{s}, \epsilon_{t}\right)=0$ para $s \neq t\left(\sigma^{2}>0\right.$ também é um parâmetro desconhecido do modelo).

Neste caso a representação de espaço de estados 3.33 e 3.34 adequada para a estimação adaptativa dos parâmetros do vetor b pode ser escrita como

$$
\begin{gathered}
\mathbf{b}_{t+1}=\mathbf{b}_{t}, \\
y_{t}=\mathbf{z}_{t} \mathbf{b}_{t}+\boldsymbol{\epsilon}_{t},
\end{gathered}
$$

em que $\mathbf{x}_{t}=\mathbf{b}_{t}, \mathbf{v}_{t}=\boldsymbol{\epsilon}_{t}, \boldsymbol{\Phi}_{t}=\mathbf{I}, \boldsymbol{\Gamma}_{t}=0, \mathbf{M}_{t}=\mathbf{z}_{t}^{\prime}, \mathbf{Q}_{t}=0, \mathbf{R}_{t}=\sigma^{2}$. Assim, as relações entre 3.40 e 3.41 usando a equação 3.42 têm a forma

$$
\begin{gathered}
\hat{\mathbf{b}}_{t+1}=\hat{\mathbf{b}}_{t}+\sigma^{-2} \mathbf{P}_{t+1} \mathbf{z}_{t+1}\left(y_{t+1}-\mathbf{z}_{t+1}^{\prime} \hat{\mathbf{b}}_{t}\right), \\
\mathbf{P}_{t+1}=\mathbf{P}_{t}-\left(\mathbf{z}_{t+1}^{\prime} \mathbf{P}_{t} \mathbf{z}_{t+1}+\sigma^{2}\right)^{-1} \mathbf{P}_{t} \mathbf{z}_{t+1} \mathbf{z}_{t+1}^{\prime} \mathbf{P}_{t} .
\end{gathered}
$$

Uma vez que o parâmetro $\sigma^{2}$ é desconhecido pode-se colocar

$$
\mathbf{V}_{t}=\sigma^{-2} \mathbf{P}_{t}
$$

e reescrevemos 3.49 e 3.50 na forma

$$
\hat{\mathbf{b}}_{t+1}=\hat{\mathbf{b}}_{t}+\mathbf{V}_{t+1} \mathbf{z}_{t+1}\left(y_{t+1}-\mathbf{z}_{t+1}^{\prime} \hat{\mathbf{b}}_{t}\right)
$$




$$
\mathbf{V}_{t+1}=\mathbf{V}_{t}-\left(\mathbf{z}_{t+1}^{\prime} \mathbf{V}_{t} \mathbf{z}_{t+1}+1\right)^{-1} \mathbf{V}_{t} \mathbf{z}_{t+1} \mathbf{z}_{t+1}^{\prime} \mathbf{V}_{t}
$$

que são as fórmulas do método dos mínimos quadrados recursivos para o modelo 3.46. E a estimativa adaptativa do parâmetro $\sigma^{2}$ pode ser obtida usando a relação

$$
\sigma_{t}^{2}=\frac{1}{t-\gamma} \sum_{i=1}^{t}\left(\mathbf{y}_{i}-\mathbf{z}_{i}^{\prime} \hat{\mathbf{b}}_{t}\right)^{2}
$$

e a estimativa adaptatvia de $\sigma^{2}$ :

$$
\hat{\sigma}_{t+1}^{2}=\frac{1}{t+1-\gamma}\left[(t-\gamma) \hat{\sigma}_{t}^{2}+\left(\mathbf{z}_{t+1}^{\prime} \mathbf{V}_{t} \mathbf{z}_{t+1}+1\right)^{-1}\left(y_{t+1}-\mathbf{z}_{t+1}^{\prime} \hat{\mathbf{b}}_{t}\right)^{2}\right]
$$

A matriz $\hat{\mathbf{P}}_{t+1}=\hat{\sigma}_{t+1}^{2} \mathbf{V}_{t+1}$ pode ser tomada como a estimativa da matriz de variância de $\hat{\mathbf{b}}_{t+1}$. Se não houver a informação priori sobre os parâmetros, pode-se escolher os valores iniciais das duas estimativas no instante $t_{0}$, por exemplo, como

$$
\hat{\mathbf{b}}_{t_{0}}=0, \mathbf{V}_{t_{0}}=c^{-1} \mathbf{I}, \hat{\sigma}_{t_{0}}^{2}=c,
$$

em que c é uma pequena constante positiva.

\subsubsection{Estimação em processo ARMA multivariado}

O modelo STARMA é um caso específico do modelo VARMA, podemos escrever o STARMA como

$$
\begin{aligned}
\mathbf{y}_{t} & =\sum_{k=1}^{p} \sum_{l=0}^{\lambda_{k}} \phi_{k l} \mathbf{W}^{l} \mathbf{y}_{t-k}-\sum_{k=1}^{q} \sum_{l=0}^{m_{k}} \theta_{k l} \mathbf{W}^{l} \boldsymbol{\epsilon}_{t-k}+\boldsymbol{\epsilon}_{t} \\
& =\sum_{l=0}^{\lambda_{1}} \phi_{1 l} \mathbf{W}^{l} \mathbf{y}_{t-1}+\ldots+\sum_{l=0}^{\lambda_{p}} \phi_{p l} \mathbf{W}^{l} \mathbf{y}_{t-p}-\sum_{l=0}^{m_{1}} \theta_{1 l} \mathbf{W}^{l} \boldsymbol{\epsilon}_{t-1} \\
& -\ldots-\sum_{l=0}^{m_{q}} \theta_{q l} \mathbf{W}^{l} \boldsymbol{\epsilon}_{t-q}+\boldsymbol{\epsilon}_{t} \\
& =\boldsymbol{\Phi}_{1} \mathbf{y}_{t-1}+\ldots+\boldsymbol{\Phi}_{p} \mathbf{y}_{t-p}-\mathbf{\Theta}_{1}^{*} \boldsymbol{\epsilon}_{t-1}-\ldots-\boldsymbol{\Theta}_{q}^{*} \boldsymbol{\epsilon}_{t-q}+\boldsymbol{\epsilon}_{t} \\
& =\boldsymbol{\Phi}_{1} \mathbf{y}_{t-1}+\ldots+\boldsymbol{\Phi}_{p} \mathbf{y}_{t-p}+\boldsymbol{\Theta}_{1} \boldsymbol{\epsilon}_{t-1}+\ldots+\boldsymbol{\Theta}_{q} \boldsymbol{\epsilon}_{t-q}+\boldsymbol{\epsilon}_{t}
\end{aligned}
$$

que é um $\operatorname{VARMA}(\mathrm{p}, \mathrm{q})$., em que $\boldsymbol{\Phi}_{1}, \ldots, \boldsymbol{\Phi}_{p}, \boldsymbol{\Theta}_{1}, \ldots, \boldsymbol{\Theta}_{q}$ são as matrizes de parâmetros de dimensão (n,n) e $\boldsymbol{\epsilon}_{t}$ é o ruído branco n-dimensional, com $\mathrm{E}\left(\boldsymbol{\epsilon}_{t}\right)=0, \operatorname{Var}\left(\boldsymbol{\epsilon}_{t}\right)=\Sigma>$ $0, \operatorname{Cov}\left(\boldsymbol{\epsilon}_{s}, \boldsymbol{\epsilon}_{t}\right)=0$ para $s \neq t$. Denotamos 


$$
\mathbf{b}=\left(\begin{array}{c}
\operatorname{vec} \boldsymbol{\Phi}_{1} \\
\vdots \\
\operatorname{vec} \boldsymbol{\Phi}_{p} \\
\operatorname{vec} \boldsymbol{\Theta}_{1} \\
\vdots \\
\operatorname{vec} \boldsymbol{\Theta}_{q}
\end{array}\right)
$$

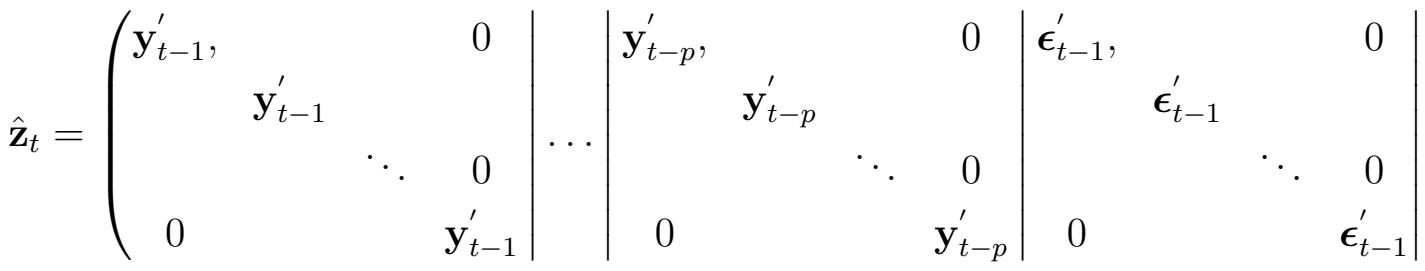

$$
\begin{aligned}
& \left.\ldots \mid \begin{array}{cccc}
\boldsymbol{\epsilon}_{t-q}^{\prime}, & & & 0 \\
& \boldsymbol{\epsilon}_{t-q}^{\prime} & & \\
& & \ddots & 0 \\
0 & & & \boldsymbol{\epsilon}_{t-q}^{\prime}
\end{array}\right) \\
& \hat{\boldsymbol{\epsilon}}_{t}=\mathbf{y}_{t}-\hat{\mathbf{z}}_{t} \hat{\mathbf{b}}_{t} .
\end{aligned}
$$

A dimensão do vetor b é $\left(n^{2}(p+q), 1\right)$ e da matriz $\hat{\mathbf{z}}_{t}$ é $\left(n, n^{2}(p+q)\right)$.

As fórmulas de estimação adaptativa para o sistema

$$
\begin{gathered}
\mathbf{b}_{t+1}=\mathbf{b}_{t}, \\
\mathbf{y}_{t}=\hat{\mathbf{z}}_{t} \mathbf{b}_{t}+\boldsymbol{\epsilon}_{t}
\end{gathered}
$$

têm a seguinte forma

$$
\begin{gathered}
\hat{\mathbf{b}}_{t+1}=\hat{\mathbf{b}}_{t}+\mathbf{P}_{t+1} \hat{\mathbf{z}}_{t+1}^{\prime} \hat{\mathbf{\Sigma}}_{t}^{-1}\left(\mathbf{y}_{t+1}-\hat{\mathbf{z}}_{t+1} \hat{\mathbf{b}}_{t}\right), \\
\mathbf{P}_{t+1}=\mathbf{P}_{t}-\mathbf{P}_{t} \hat{\mathbf{z}}_{t+1}^{\prime}\left(\hat{\mathbf{z}}_{t+1} \mathbf{P}_{t} \mathbf{z}_{t+1}^{\prime}+\hat{\mathbf{\Sigma}}_{t}\right)^{-1} \mathbf{z}_{t+1}^{\prime} \mathbf{P}_{t} \\
\hat{\mathbf{\Sigma}}_{t+1}=\frac{1}{t+1-n^{2}(p+q)}\left\{\left[t-n^{2}(p+q)\right] \hat{\mathbf{\Sigma}}_{t}+\left(\mathbf{y}_{t+1}-\hat{\mathbf{z}}_{t+1} \hat{\mathbf{b}}_{t+1}\right)\left(\mathbf{y}_{t+1}-\hat{\mathbf{z}}_{t+1} \hat{\mathbf{b}}_{t+1}\right)^{\prime}\right\}, \\
\hat{\boldsymbol{\epsilon}}_{t+1}=\mathbf{y}_{t+1}-\hat{\mathbf{z}}_{t+1} \hat{\mathbf{b}}_{t+1} .
\end{gathered}
$$




\subsection{Diagnóstico}

Nesta seção, apresentamos o diagnóstico da classe de modelos STARMA, isto é confirmar se o modelo identificado é um modelo adequado para descrever os dados de interesse. Para isso, deve-se realizar uma análise residual e observar se os resíduos são ruído branco, ou seja, devem ser independentes com distribuição normal multivariada com média zero e matriz de covariância $\sigma \mathbf{I}_{N T}$.

Para testar se os resíduos são independentes, basta observar se todas as suas autocorrelações de espaço-temporal de defasagens espaciais diferentes de zero devem ser iguais a zero, ou seja, ficam dentro do intervalo de confiança. Para obter o intervalo de confianção para STFAC é fundamental calcuar a variância da STFAC. Conforme Pfeifer e Deutsch (1981a)

$$
\operatorname{var}\left(\hat{\rho}_{l 0}(s)\right) \approx \frac{1}{N(T-S)},
$$

em que $\hat{\rho}_{l 0}(s)$ é a STFAC amostral do resíduo do modelo ajustado.

\subsection{Previsão}

A previsão para modelos STARMA é semelhante aos modelos univariados de BoxJenkins apresentados no capítulo 2, para STARMA temos o caso multivariado. Para prever os valores de $\mathbf{Z}(t+h), h \geq 1$ é necessário utilizar as observações de $\mathbf{Z}(t), \mathbf{Z}(t-1), \ldots$, segundo Morettin e Toloi (2006), o momento t é chamado de origem das previsãoes, e h, a horizonte. Assim, a previsão de $\mathbf{Z}(t+h)$ denotada por $\hat{\mathbf{Z}}_{t}(h)$ assumindo que o processo $\mathbf{Z}(t)$ é estacionário e invertível, é apresentada por

$$
\hat{\mathbf{Z}}_{t}(h)=E[\mathbf{Z}(t+h) \mid \mathbf{Z}(t), \mathbf{Z}(t-1), \ldots]
$$

Para um modelo de STARMA, as previsões de origem t e horizonte h são dadas por

$$
\hat{\mathbf{Z}}_{t}(h)=E\left[\sum_{k=1}^{p} \sum_{l=0}^{\lambda_{k}} \hat{\phi}_{k l} \mathbf{W}^{(l)} \mathbf{Z}(t+h-k)-\sum_{k=1}^{q} \sum_{l=0}^{m_{k}} \hat{\theta}_{k l} \mathbf{W}^{(l)} \boldsymbol{\epsilon}(t+h-k)+\boldsymbol{\epsilon}(t+h)\right]
$$

considerando que

$$
\begin{gathered}
E[\mathbf{Z}(t+s)]=\hat{\mathbf{Z}}_{t}(s), \quad s>0, \\
E[\mathbf{Z}(t+s)]=\mathbf{Z}(t+s), \quad s \leq 0, \\
E[\boldsymbol{\epsilon}(t+s)]=0, \quad s>0, \\
E[\boldsymbol{\epsilon}(t+s)]=\boldsymbol{\epsilon}(t+s), \quad s \leq 0 .
\end{gathered}
$$


Note que $\boldsymbol{\epsilon}(t+s)=\mathbf{Z}(t+s)-\hat{\mathbf{Z}}(t+s), \hat{\mathbf{Z}}(t+s)$ é o valor ajustado da $\mathbf{Z}(t+s)$ do modelo. 


\section{Capítulo 4 \\ Estudo de Simulação}

No capítulo anterior, foram apresentadas as definições e os conceitos sobre a classe dos modelos STARMA, vemos que existem vários métodos para construir a matriz de pesos espaciais $\mathbf{W}^{(l)}$, sendo 1 a ordem de vizinhança. Para Pfeifer e Deutsch (1980a), a maneira para determinar a matriz de pesos é baseada na contiguidade espacial. Para Rao e Antunes (2004) a escolha da matriz depende do pesquisador e do conhecimento sobre o tipo de estudo. Na literatura, há poucos estudos que investigam se existe a melhor forma da matriz de pesos espaciais no contexto de melhor ajuste de modelos STARMA.

O objetivo deste capítulo é comparar as estruturas de vizinhanças espaciais ou matrizes de pesos espaciais da classe de modelos autorregressivos e de médias móveis espaço-temporais (STARMA). Foram realizadas simulações utilizando vários modelos de covariância espaço-temporal para comparar diferentes estruturas de construção da matriz de pesos espaciais com a finalidade de identificar a melhor matriz. O Filtro de Kalman será adotado para a estimação dos modelos STARMA utilizando o pacote starma do software $R$.

\subsection{Modelos de Covariância Espaço-Temporal}

Conforme Sherman (2011), derivando do cenário puramente espacial para o caso espaço-temporal, temos a variável $\left\{Z(\mathbf{s}, t): \mathbf{s} \in \mathbb{R}^{d}, t \in \mathbb{R}\right\}$, d a dimensão do espaço; e é definido $C(\mathbf{h}, u)$ a covariância entre duas variáveis espaço-temporal com defasagens espaciais $\mathbf{h} \in \mathbb{R}^{d}$ e defasagens temporais $\mathrm{u}$, isto é

$$
\operatorname{Cov}[Z(\mathbf{s}+\mathbf{h}, t+u), Z(\mathbf{s}, t)]=\operatorname{Cov}[Z(\mathbf{h}, u), Z(\mathbf{0}, 0)]=: C(\mathbf{h}, u)
$$

A estimativa das estruturas da covariância espaço-temporal pode ser altamente complicada a menos que sejam utilizadas suposições simplificadoras. Focamos aqui no processo estacionário de segunda ordem ou fracamente estacionário com a função de covariância espaço-temporal $C(\mathbf{h}, u)$.

Segundo Gelfand et al. (2010), a função covariância espaço-temporal é definida como 
separável se consegue ser escrita pelo produto da função de covariância puramente espacial e puramente temporal $C_{S}$ e $C_{T}$ de forma que

$$
C(\mathbf{h}, u)=C_{S}(\mathbf{h}) \cdot C_{T}(u)
$$

para todas as $(\mathbf{h}, u) \in \mathbb{R}^{d} \times \mathbb{R}$.

A função covariância espaço-temporal é definida como totalmente simétrica se

$$
C(\mathbf{h}, u)=C(\mathbf{h},-u)=C(-\mathbf{h}, u)=C(-\mathbf{h},-u) .
$$

Segundo Sherman (2011), as funções separáveis de covariância espaço-temporal são necessariamente totalmente simétricas, mas não vice versa. Uma subcategorização importante de todos os modelos espaço-temporal é aqueles com funções de covariância separáveis e funções de covariância não separáveis.

Um típico caso do modelo de covariância espaço-temporal separável é o modelo de covariância espaço-temporal exponencial. A covariância espaço-temporal exponencial com o parâmetro de escala $\sigma^{2}$, o parâmetro de decaimento da curva espacial $v_{s}$, e com a correlação temporal que é um processo autorregressivo de ordem um com o parâmetro positivo $\rho=\exp \left(-v_{t}\right)$, é não negativa definida dada por

$$
C(\mathbf{h}, u, \theta)=\sigma^{2} \exp \left(-v_{s}\|\mathbf{h}\|\right) \exp \left(-v_{t}|u|\right)
$$

em que $\theta=\left(\sigma^{2}>0, v_{s}>0\right.$ e $\left.v_{t}>0\right)$.

Pela equação 4.4, se fixamos qualquer dois lags espaciais $\mathbf{h}_{1}$ e $\mathbf{h}_{2}$, temos $C\left(\mathbf{h}_{1}, u, \theta\right)=$ $C_{1} \exp \left(-v_{t}|u|\right) \operatorname{com} C_{1}=\sigma^{2} \exp \left(-v_{s}\left\|\mathbf{h}_{1}\right\|\right)$ e $C\left(\mathbf{h}_{2}, u, \theta\right)=C_{2} \exp \left(-v_{t}|u|\right) \operatorname{com} C_{2}=$ $\sigma^{2} \exp \left(-v_{s}\left\|\mathbf{h}_{2}\right\|\right)$. Isso mostra que as duas covariâncias temporais têm a mesma forma e diferem apenas por um fator de escala constante.

Segundo Sherman (2011) e Cressie e Huang (1999), o modelo de covariância espaçotemporal exponencial é relativamente parcimonioso e facilmente interpretável, porém muitos fenômenos físicos não satisfazem esta separabilidade. Por isso, é apropriado considerar as covariâncias espaço-temporais não separáveis.

Para o caso de modelo de covariância espaço-temporal não separável, segundo Sherman (2011), o modelo não separável de Gneiting é dado por

$$
C(\mathbf{h}, u, \theta)=\frac{\sigma^{2}}{\left(|u|^{2 \gamma}+1\right)^{\tau}} \exp \left[\frac{-c\|\mathbf{h}\|^{2 \gamma}}{\left(|u|^{2 \gamma}+1\right)^{\beta \gamma}}\right] .
$$

Em que, $\tau$ determina a suavidade da correlação temporal, enquanto $\gamma \in(0,1]$ determina a suavidade da correlação espacial, c regula a intensidade, a força da correlação espacial, e $\beta \in[0,1)$ controla a força da interação entre espaço e tempo. Quando $\gamma=1$ corresponde à função da covariância gaussiana, e $\gamma=\frac{1}{2}$ corresponde à distribuição expo- 
nencial. Valores menores refletem menos lisura, enquanto valores maiores refletem a mais suavidade.

Notamos que quando $\beta=0$, a covariância espaço-temporal de Gneiting é o produto de dois fatores, a função de espaço e a função de tempo, ou seja, um modelo de covariância separável. Figura 4.1 mostra os contornos da função de covariância espaço-temporal de Gneiting $\operatorname{com} \beta=0,0,5$ e 0,9 .

Percebe-se que os contornos são ligeiramente convexos quando $\beta=0$, próximos a linear para $\beta=0,5$ e pouco côncavos para $\beta=0,9$. Segunda Sherman (2011), muitas vezes, essa flexibilidade refletida por $\beta$ é muito desejável para modelar uma classe maior de fenômenos espaço-temporais do que é possível sob uma suposição de separabilidade.

Mais detalhes e demonstrações de parametrização dos modelos de covariância espaçotemporal podem ser encontrados no Cressie e Huang (1999), Gneiting (2002) e Stein (2005).

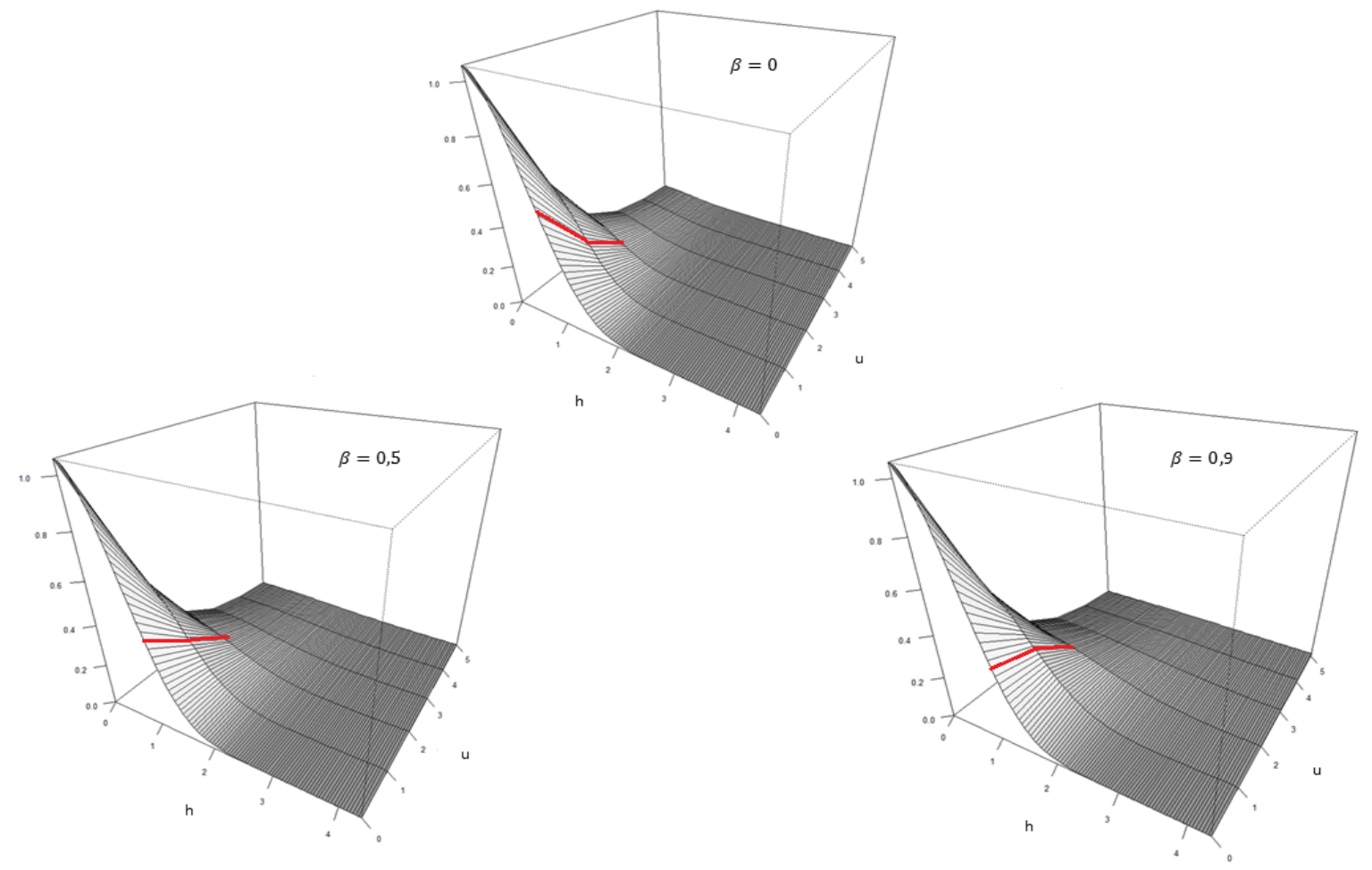

Figura 4.1: Os contornos da covariância espaço-temporal de Gneiting com $\beta=$ $0,0,5$ e 0,9 . 


\subsection{Descrição dos Processos de Simulação}

Em nosso estudo de simulação, seja $\mathrm{t}=1,2, \ldots, \mathrm{T}$, e $\mathrm{s}=s_{1}, s_{2}, \ldots, s_{N}, \mathrm{t}$ o tempo e $\mathrm{s}$ a região. Supomos que o processo espacial é fracamente estacionário, para qualquer região s e para qualquer tempo t. Isto é

i. $E[Z(\mathbf{s}, t)]=\mu \mathrm{e}$

ii. $\operatorname{Cov}[Z(\mathbf{s}+\mathbf{h}, t+u), Z(\mathbf{s}, t)]=\operatorname{Cov}[Z(\mathbf{h}, u), Z(\mathbf{0}, 0)]=: C(\mathbf{h}, u)$, para todas defasagens espaciais $\mathbf{h}$ e defasagens temporais $u$.

Para a nossa simulação, temos

$$
\left(\begin{array}{c}
X_{s_{1}}(1) \\
\vdots \\
X_{s_{1}}(T) \\
\vdots \\
X_{s_{N}}(1) \\
\vdots \\
X_{s_{N}}(T)
\end{array}\right) \sim N\left(\left(\begin{array}{c}
\mu \\
\vdots \\
\mu \\
\vdots \\
\mu \\
\vdots \\
\mu
\end{array}\right) ; \Sigma_{N T \times N T}\right)
$$

em que $\Sigma$ é a matriz de covariância espaço-temporal dada por $C(\mathbf{h}, u)$.

Utilizamos o modelo não separável de Gneiting (Sherman (2011)) para construir a dependência espaço-temporal neste estudo de simulação. Construímos 9 modelos de covariância espaço-temporal especificando diferentes valores de parâmetros do modelo de Gneiting. Fixamos $\gamma=1$ que corresponde ao processo Gaussiano, $\mathrm{c}=1, \tau=1$ e $\sigma^{2}=1$ com coeficiente de variação $\frac{\sigma}{\mu}=10 \%, 50 \%$, e $90 \%$, ou seja $\mu=10,2$ e 1,11. E para cada combinação de $\sigma$ e $\mu$, variando o parâmetro $\beta, \beta=0,0,5$ e 0,9 . Note que quando $\beta=0$ temos o modelo de covariância espaço-temporal do tipo separável exponencial.

$\mathrm{Na}$ construção das amostras, primeiramente geramos 5 e 10 pontos na região ilustrada nas Figuras 4.2 e 4.3. Estes pontos são os mesmos para todas as amostras simuladas.

Depois de ter estabelecidos os pontos $s_{1}, s_{2}, \ldots, s_{N}, \mathrm{~N}=5$ ou 10 , geramos $\mathrm{N} \times \mathrm{T}$ valores da distribuição normal multivariada com média $(\mu, \ldots, \mu)^{\prime}$ e matriz de covariância espaçotemporal $\Sigma$, notando que T é o tamanho da série de cada região, escolhemos $\mathrm{T}=100$. 


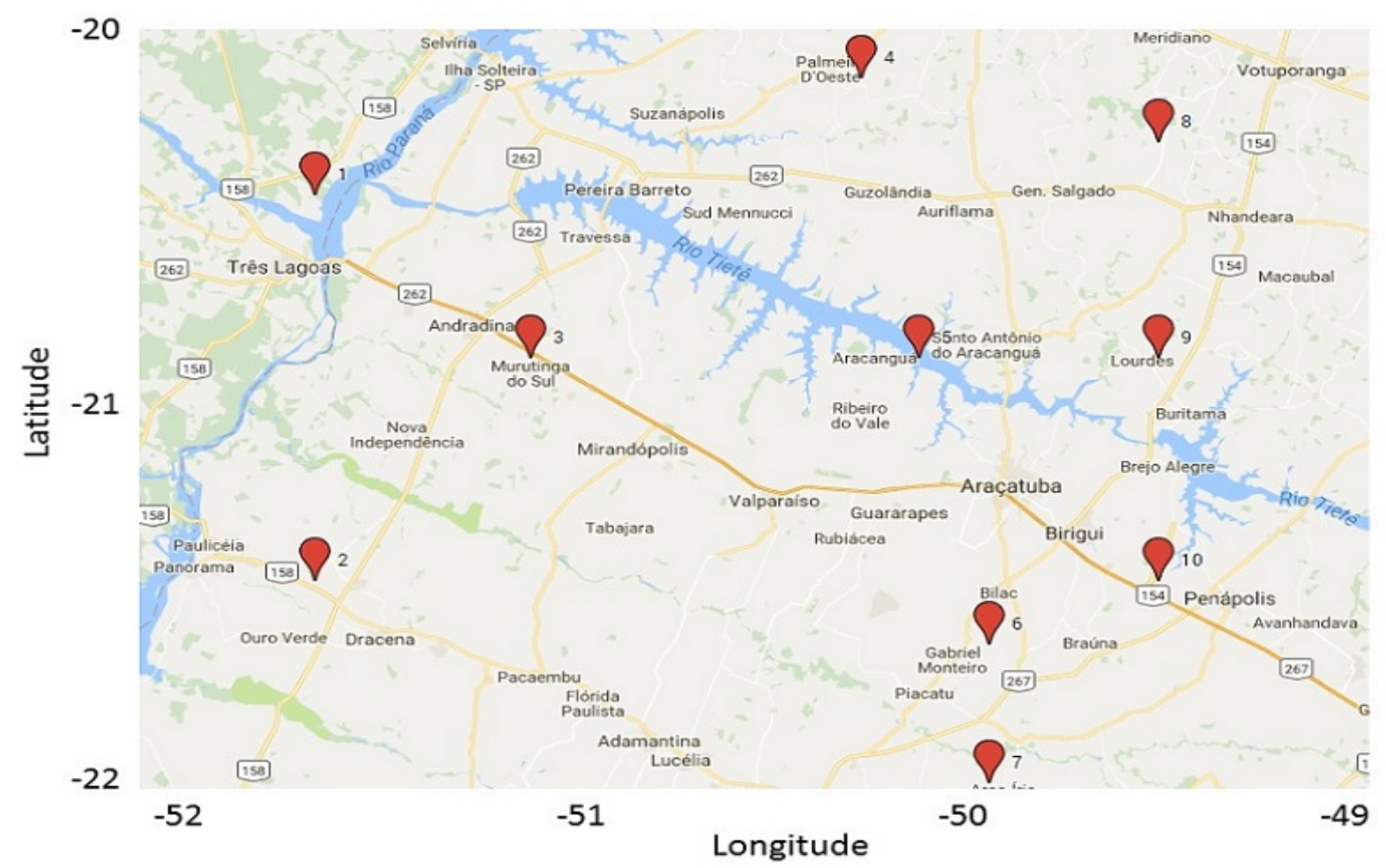

Figura 4.2: Região onde os dados foram simulados. Os 10 pontos são localidades selecionadas em simulação

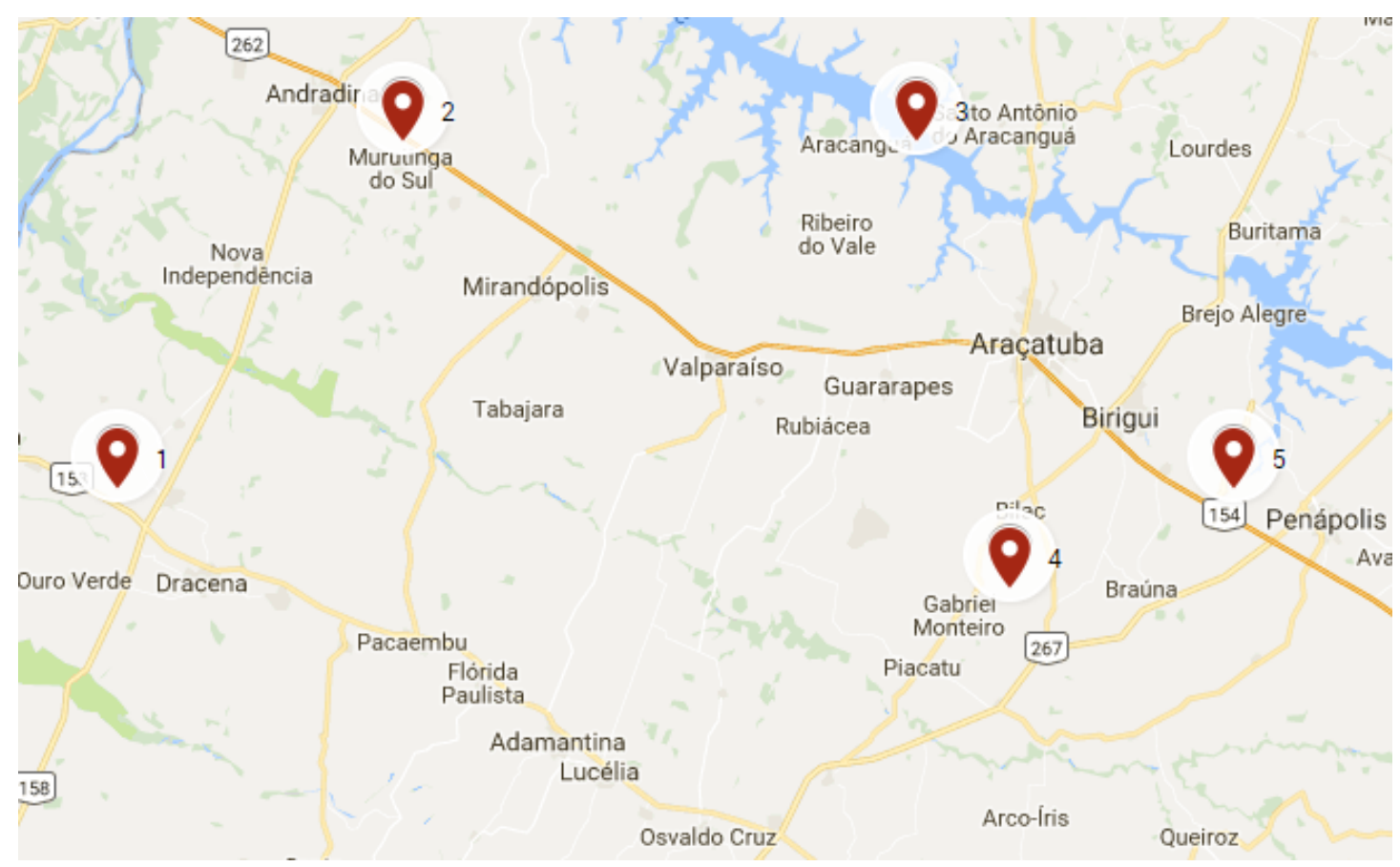

Figura 4.3: Região onde os dados foram simulados. Os 5 pontos são localidades selecionadas em simulação 


\subsection{Avaliação de Ajuste}

Foram geradas 100 amostras de séries espaços temporais para 5 e 10 regiões, utilizando todos os modelos de Gneiting mencionados anteriormente. Para cada amostra, ajustamos modelos de $\operatorname{STAR}\left(1_{1}\right)$ de uma defasagem tanto tempo quanto espaço com as sete distintas matrizes de pesos. Os sete métodos de construção da matriz espacial (veja Capítulo 3) são: a função potência da distância geodésica (grande círculo) $\frac{1}{d^{a}}, \mathrm{a}=0,5,1$ e 2; e a função exponencial da distância geodésica $e^{-a d}$, a =0,5, 1 e 2, e a contiguidade espacial definida de ordem 1. Note-se que a função potência da distância geodésica e a função exponencial quantificam a dependência local em uma única matriz de pesos e os modelos de STARMA ficarem restritos a apenas uma defasagem espacial, diferentes da contiguidade espacial que pode ter mais de uma ordem de vizinhança, e além disso, depende bastante do conhecimento do pesquisador sobre regiões em estudo.

A escolha do modelo STAR de ordem um para ajustar as séries simuladas foi feita pela propriedade dos modelos de covariância espaço-temporal, que os modelos podem ter uma correlação temporal que é um processo autorregressivo de ordem um, por exemplo o modelo separável exponencial e a sua forma geral, o modelo não separável Gneiting; além disso, durante a simulação, foram testados também STAR de ordem temporal dois, porém os parâmetros correspondentes a defasagem dois não foram significativos, portanto modelo de STAR $\left(1_{1}\right)$ foi adotado para o ajuste das séries. Para avaliar o desempenho desses métodos do cálculo da matriz, usamos a medida de comparação de ajuste Erro Quadrático Médio.

Definimos as matrizes de contiguidade espacial de ordem 1 para as 5 e 10 regiões respectivamente como

$$
\left[\begin{array}{ccccc}
0 & 1 & 0 & 0 & 0 \\
\frac{1}{2} & 0 & \frac{1}{2} & 0 & 0 \\
0 & \frac{1}{2} & 0 & 0 & \frac{1}{2} \\
0 & 0 & 0 & 0 & 1 \\
0 & 0 & \frac{1}{2} & \frac{1}{2} & 0
\end{array}\right]
$$

e 


$$
\left[\begin{array}{cccccccccc}
0 & \frac{1}{2} & \frac{1}{2} & 0 & 0 & 0 & 0 & 0 & 0 & 0 \\
0 & 0 & 1 & 0 & 0 & 0 & 0 & 0 & 0 & 0 \\
\frac{1}{2} & \frac{1}{2} & 0 & 0 & 0 & 0 & 0 & 0 & 0 & 0 \\
0 & 0 & 0 & 0 & 0 & 0 & 0 & 1 & 0 & 0 \\
0 & 0 & \frac{1}{6} & \frac{1}{6} & 0 & \frac{1}{6} & 0 & \frac{1}{6} & \frac{1}{6} & \frac{1}{6} \\
0 & 0 & 0 & 0 & \frac{1}{4} & 0 & \frac{1}{4} & 0 & \frac{1}{4} & \frac{1}{4} \\
0 & 0 & 0 & 0 & 0 & \frac{1}{2} & 0 & 0 & 0 & \frac{1}{2} \\
0 & 0 & 0 & \frac{1}{3} & \frac{1}{3} & 0 & 0 & 0 & \frac{1}{3} & 0 \\
0 & 0 & 0 & 0 & \frac{1}{4} & \frac{1}{4} & 0 & \frac{1}{4} & 0 & \frac{1}{4} \\
0 & 0 & 0 & 0 & \frac{1}{4} & \frac{1}{4} & \frac{1}{4} & 0 & \frac{1}{4} & 0
\end{array}\right] .
$$

O Erro Quadrático Médio dado por

$$
E Q M=\frac{1}{N T M} \sum_{m=1}^{M} \sum_{s=1}^{N} \sum_{t=1}^{T}\left[X_{s}(t)^{m}-\hat{X}_{s}(t)^{m}\right]^{2},
$$

sendo $\hat{X}_{s}(t)^{m}$ o valor ajustado de $X_{s}(t)^{m}$ da m-ésima amostra pelo modelo; T o tamanho da série, $\mathrm{T}=100 ; \mathrm{M}$ o número total de amostra, $\mathrm{M}=100$; e $\mathrm{N}$ o número de região, $\mathrm{N}=5$ e 10.

\subsection{Resultado de Simulação}

Foram utlizados os modelos de Gneiting fixando $\gamma=1, \mathrm{c}=1, \tau=1, \sigma^{2}=1$, e variando os parâmetros $\mu$ e $\beta$, sendo $\mu=10,2$, e 1,$11 ; \beta=0,0,5$ e 0,9 para gerar 100 amostras de séries espaço-temporais de tamanho 100 de 5 e de 10 regiões. Para cada amostra, foram ajustados os seis modelos de $\operatorname{STAR}\left(1_{1}\right)$ com as matrizes de pesos espaciais $\frac{1}{d^{0,5}}, \frac{1}{d}, \frac{1}{d^{2}}, e^{-0,5 d}, e^{-d}, e^{-2 d}$ e a contiguidade espacial; e por fim, calculamos os EQM (Erro Quadrático Médio) dos modelos ajustados. A comparação dos EQM das matrizes de pesos espaciais pode ser visualizada pelas Figuras de 4.4 até 4.12. As Tabelas 4.1 e 4.2 foram feitas para resumir os resultados da comparação. E nas Tabelas 4.3 e 4.4 se encontram os tempos decorridos para gerar as séries simuladas e as estimações dos modelos de STAR de ordem 1 com as sete matrizes diferentes de pesos.

\subsection{Conclusão de Simulação}

Pelas Figuras 4.4 até 4.12, percebe-se que entre os cálculos das matrizes espaciais $\frac{1}{d^{a}}$, aparentemente a medida EQM aumenta quando a potência a aumenta. A matriz de contiguidade espacial apresenta o maior EQM, e as $e^{-a d}$ mostram os menores EQM. 
Entre os pesos espaciais do tipo $e^{-a d}$, quase não há diferenças notáveis de EQM que podem ser visualizados diretamente pelos gráficos. Entretanto pelas Tabelas 4.1 e 4.2, foram detectados que o $e^{-0,5 d}$ e o $e^{-d}$ possuem performances semelhantes em relação à EQM qualidade de ajuste do modelo.

Tanto pelos gráficos quanto pelas tabelas, observamos que os efeitos dos distintos cálculos das matrizes espaciais sobre as qualidades de ajuste do modelo não mudam com a quantidade de regiões simulados. As matrizes do tipo $e^{-a d}$ mostram uma estabilidade em relação à medida de ajuste dos modelos espaços temporais por não terem diferenças relevantes entre elas, ao contrário das matrizes $\frac{1}{d^{a}}$ que têm uma variabilidade de EQM maior. Tanto para as 5 regiões quanto para as 10 regiões, a matriz de contiguidade mostrou a pior performance nas simulações, lembrando que a matriz de contiguidade é definida de acordo com a habilidade do pesquisador de forma subjetiva.

Pelas Tabelas 4.3 e 4.4 observamos que os tempos de simulações utilizando os modelos de Gneiting com $\beta=0,5$ são mais demorados. O tempo médio para executar as simulações para 5 regiões é 5 vezes menor do que o tempo para 10 regiões. E essa variação de tempo está associada com o tamanho da matriz de covariância espaço-temporal $\Sigma_{N T \times N T}$, que fica maior quando aumentamos a quantidade de regiões de estudo. Aparentemente existe uma pequena diferença entre o tempo médio de estimação do modelo STAR com a matriz espacial do tipo $\frac{1}{d^{a}}, e^{-a d}$ e a contiguidade espacial. Em média para ambas quantidades de região, o tempo médio de estimação do modelo STAR utilizando as matrizes $\frac{1}{d^{a}}$ é maior do que as $e^{-a d}$ e a contiguidade. E lembrando que o tempo de estimação do modelo com a matriz de contiguidade espacial pode aumentar quando aumenta a ordem de vizinhanças da matriz, pois a quantidade da matriz para a participação da estimação do modelo é proporcional à sua ordem de vizinhanças. Neste estudo, temos apenas uma matriz de contiguidade espacial por ter definida a ordem das vizinhanças como um. 
5 Regiões

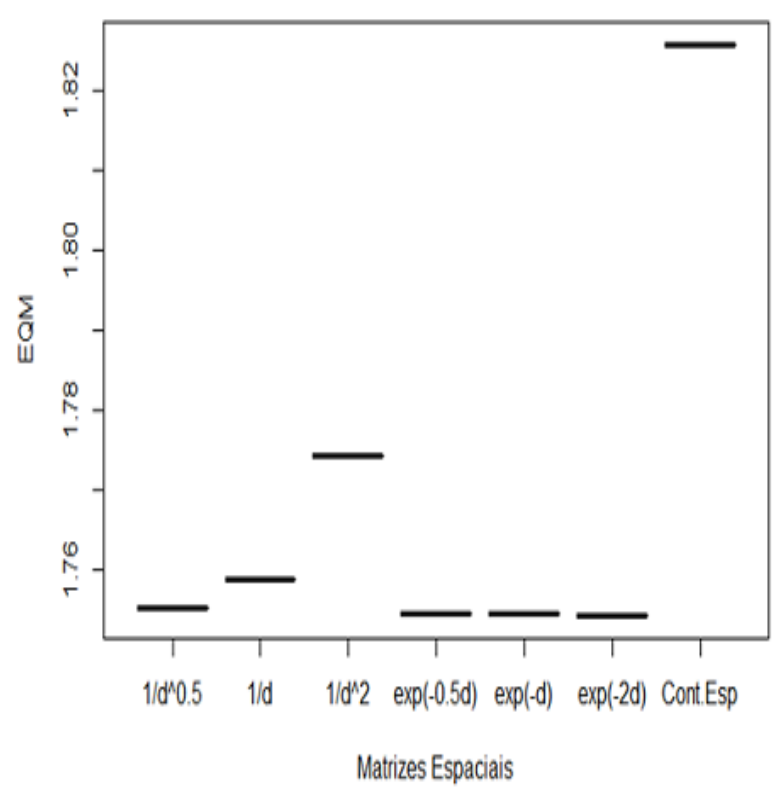

10 Regiōes

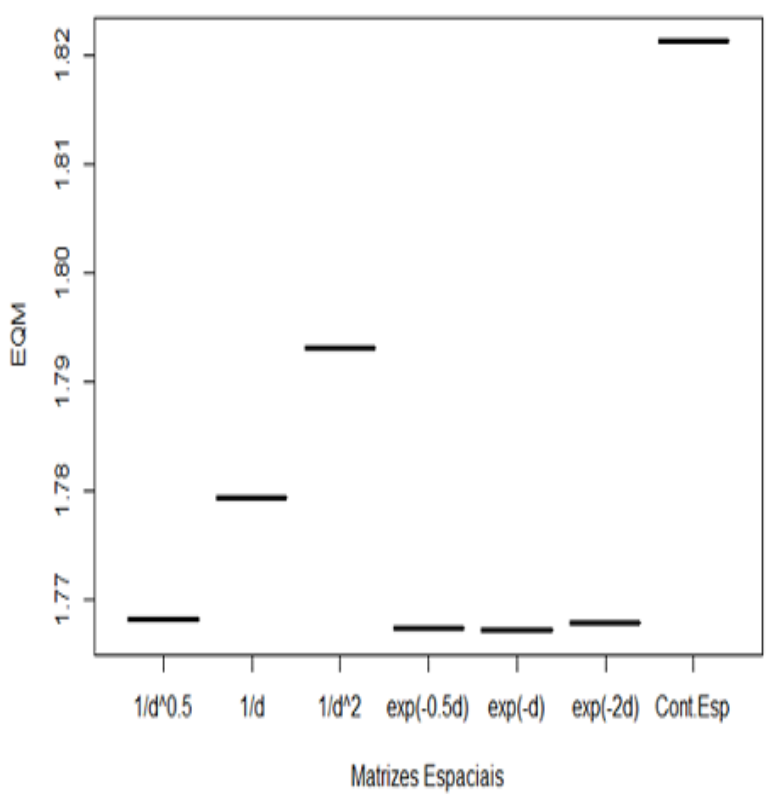

Figura 4.4: Simulação das séries pelo Gneiting $\mu=10$ e $\beta=0$; EQM calculados ajustando os modelos $\operatorname{STAR}\left(1_{1}\right)$ com as matrizes de pesos espaciais $\frac{1}{d^{0,5}}, \frac{1}{d}, \frac{1}{d^{2}}, e^{-0,5 d}, e^{-d}, e^{-2 d} \mathrm{e}$ contiguidade espacial de ordem 1 para 5 e 10 regiões.
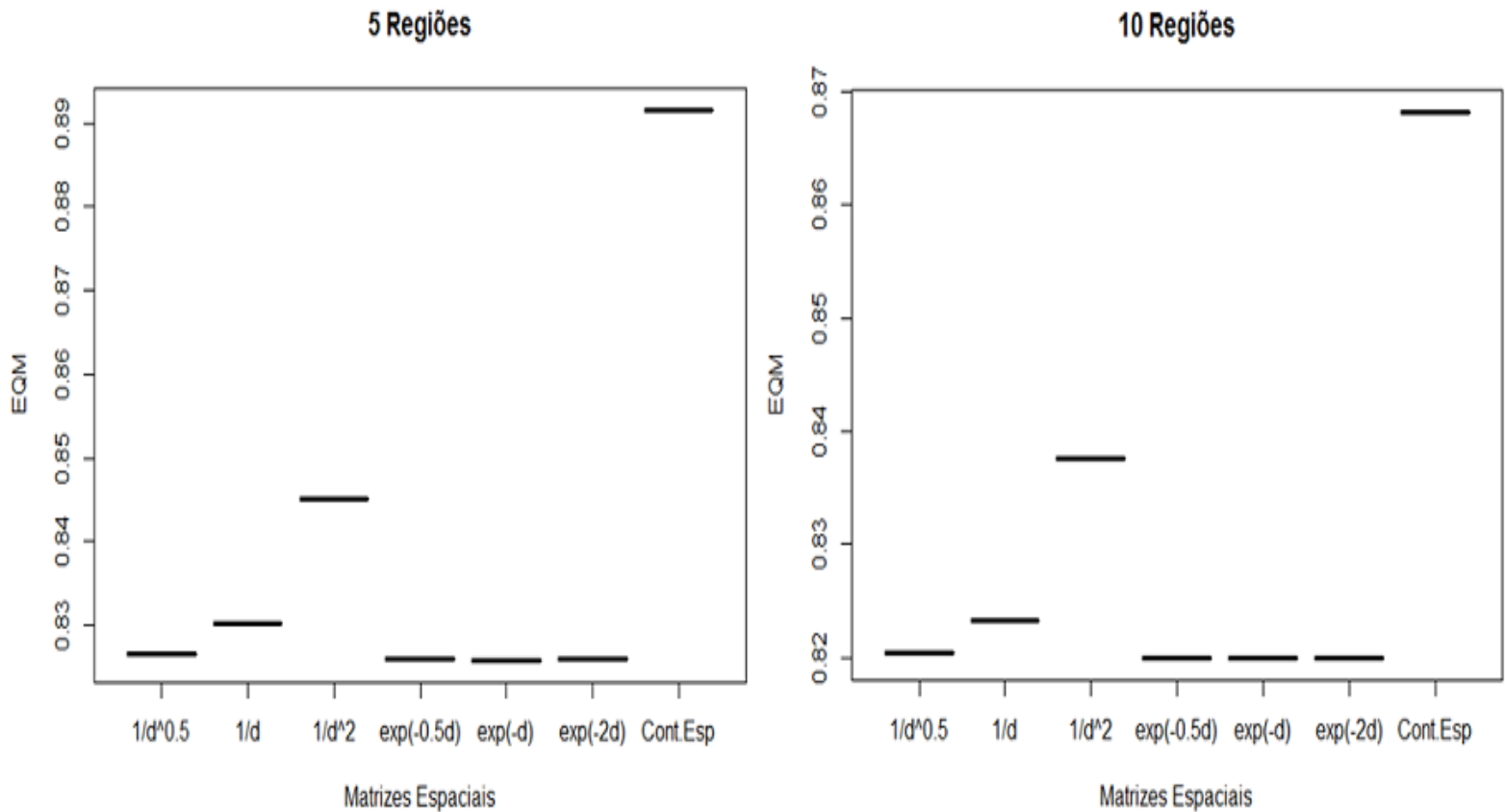

Figura 4.5: Simulação das séries pelo Gneiting $\mu=2$ e $\beta=0$; EQM calculados ajustando os modelos $\operatorname{STAR}\left(1_{1}\right)$ com as matrizes de pesos espaciais $\frac{1}{d^{0,5}}, \frac{1}{d}, \frac{1}{d^{2}}, e^{-0,5 d}, e^{-d}, e^{-2 d} \mathrm{e}$ contiguidade espacial de ordem 1 para 5 e 10 regiões. 

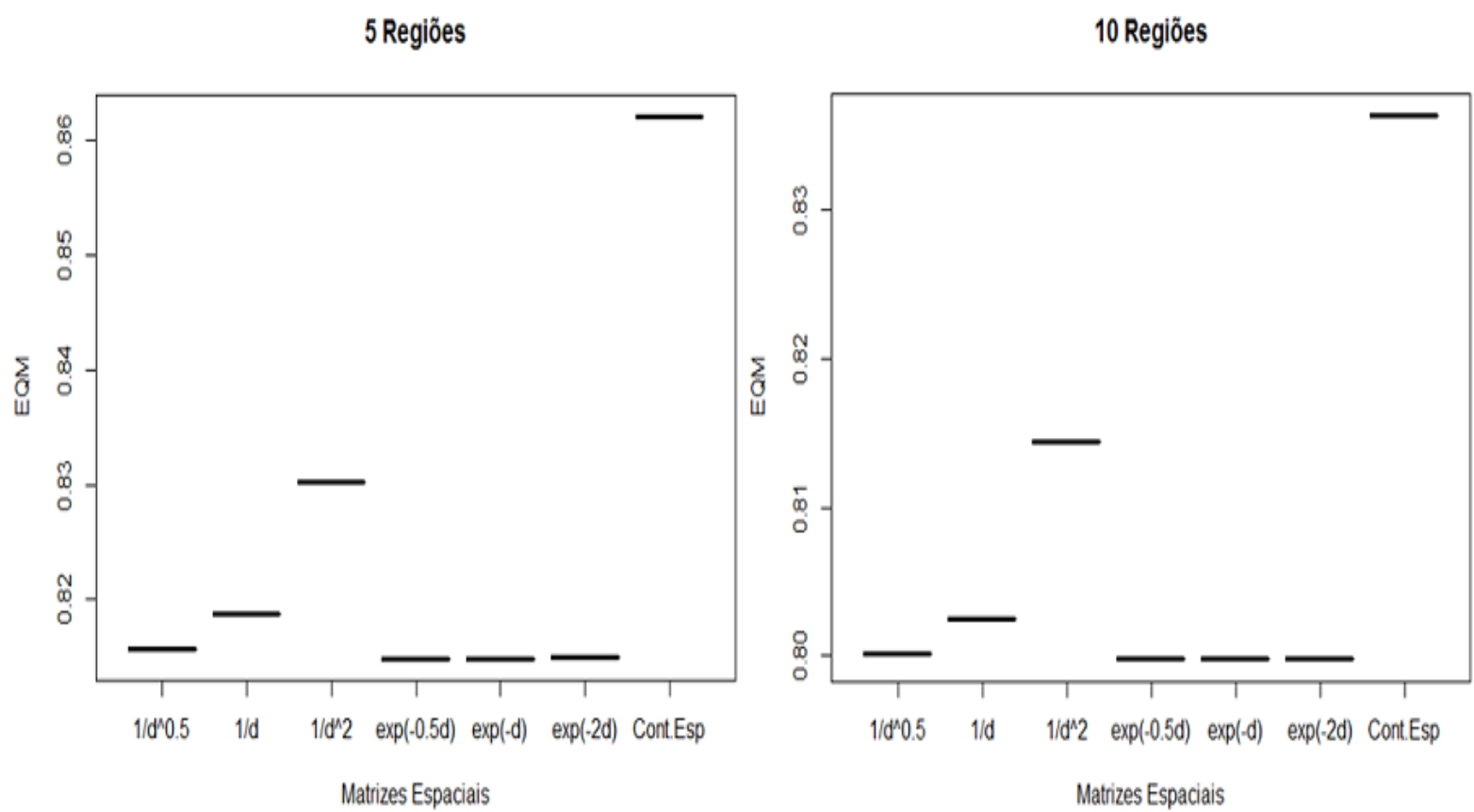

Figura 4.6: Simulação das séries pelo Gneiting $\mu=1,11$ e $\beta=0$; EQM calculados ajustando os modelos $\operatorname{STAR}\left(1_{1}\right)$ com as matrizes de pesos espaciais $\frac{1}{d^{0,5}}, \frac{1}{d}, \frac{1}{d^{2}}, e^{-0,5 d}$, $e^{-d}, e^{-2 d}$ e contiguidade espacial de ordem 1 para 5 e 10 regiões.

5 Regiões

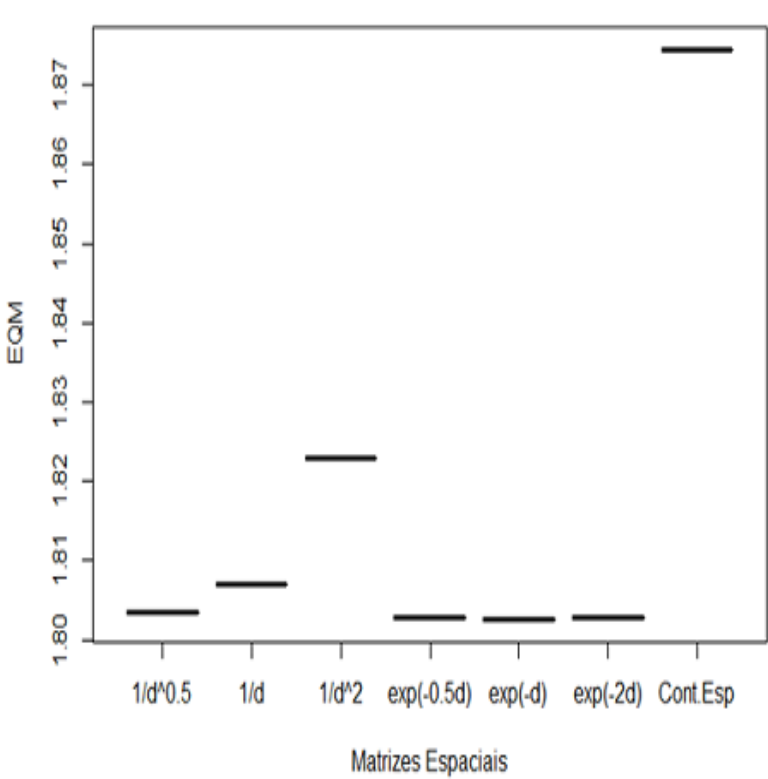

10 Regiões

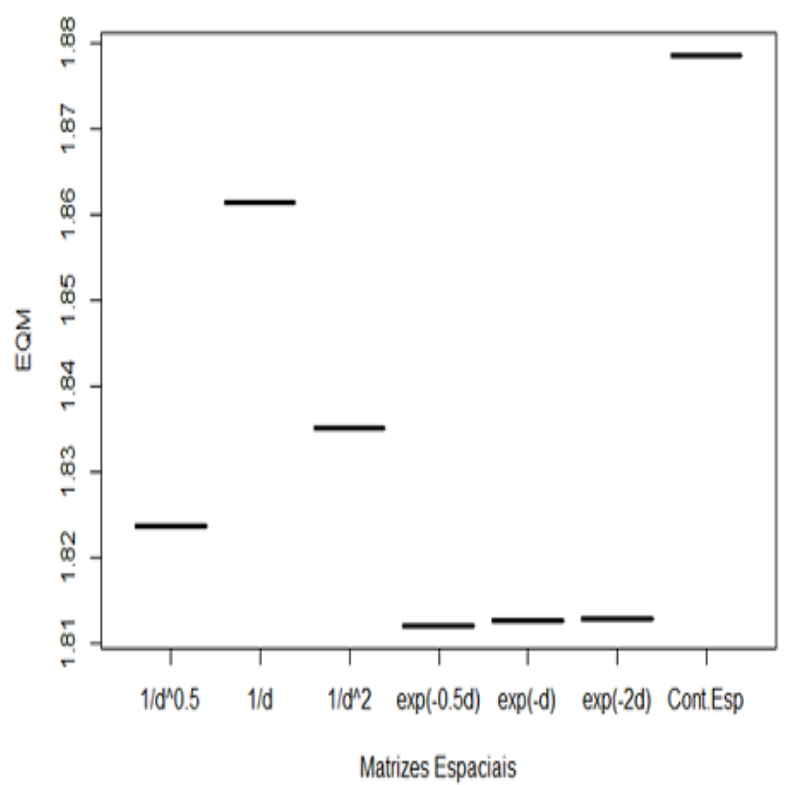

Figura 4.7: Simulação das séries pelo Gneiting $\mu=10$ e $\beta=0,5$; EQM calculados ajustando os modelos $\operatorname{STAR}\left(1_{1}\right)$ com as matrizes de pesos espaciais $\frac{1}{d^{0,5}}, \frac{1}{d}, \frac{1}{d^{2}}, e^{-0,5 d}$, $e^{-d}, e^{-2 d}$ e contiguidade espacial de ordem 1 para 5 e 10 regiões. 
5 Regiöes

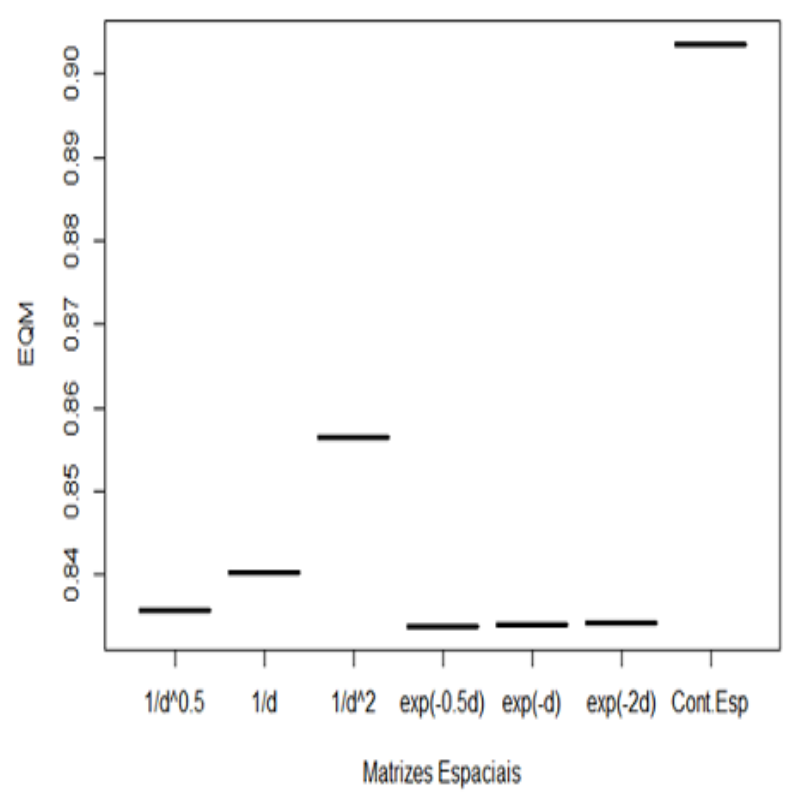

10 Regiōes

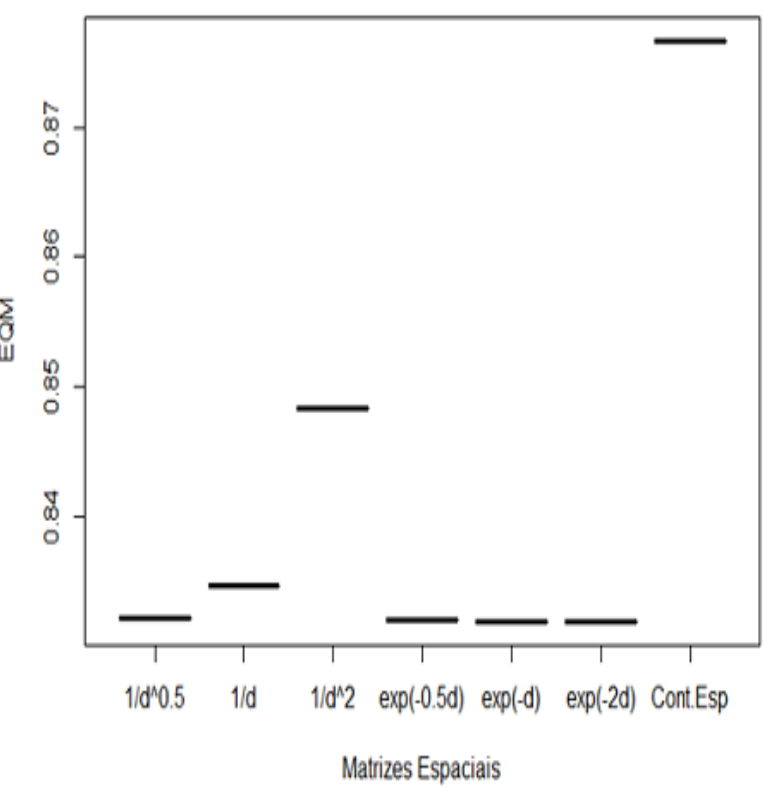

Figura 4.8: Simulação das séries pelo Gneiting $\mu=2$ e $\beta=0,5$; EQM calculados ajustando os modelos $\operatorname{STAR}\left(1_{1}\right)$ com as matrizes de pesos espaciais $\frac{1}{d^{0,5}}, \frac{1}{d}, \frac{1}{d^{2}}, e^{-0,5 d}, e^{-d}$, $e^{-2 d}$ e contiguidade espacial de ordem 1 para 5 e 10 regiões.

5 Regiões

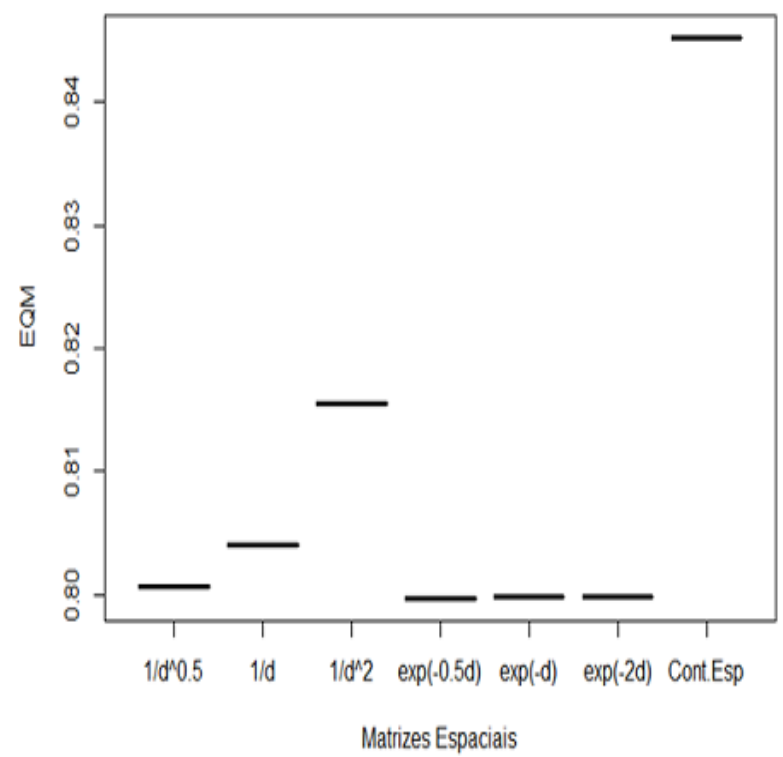

10 Regiões

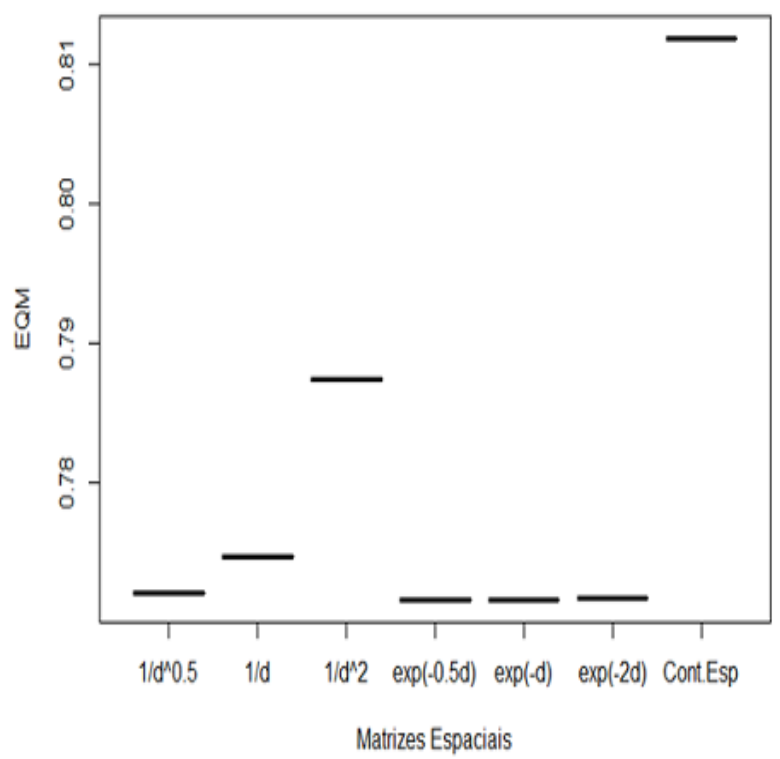

Figura 4.9: Simulação das séries pelo Gneiting $\mu=1,11$ e $\beta=0,5$; EQM calculados ajustando os modelos $\operatorname{STAR}\left(1_{1}\right)$ com as matrizes de pesos espaciais $\frac{1}{d^{0,5}}, \frac{1}{d}, \frac{1}{d^{2}}, e^{-0,5 d}$, $e^{-d}, e^{-2 d}$ e contiguidade espacial de ordem 1 para 5 e 10 regiões. 
5 Regiöes

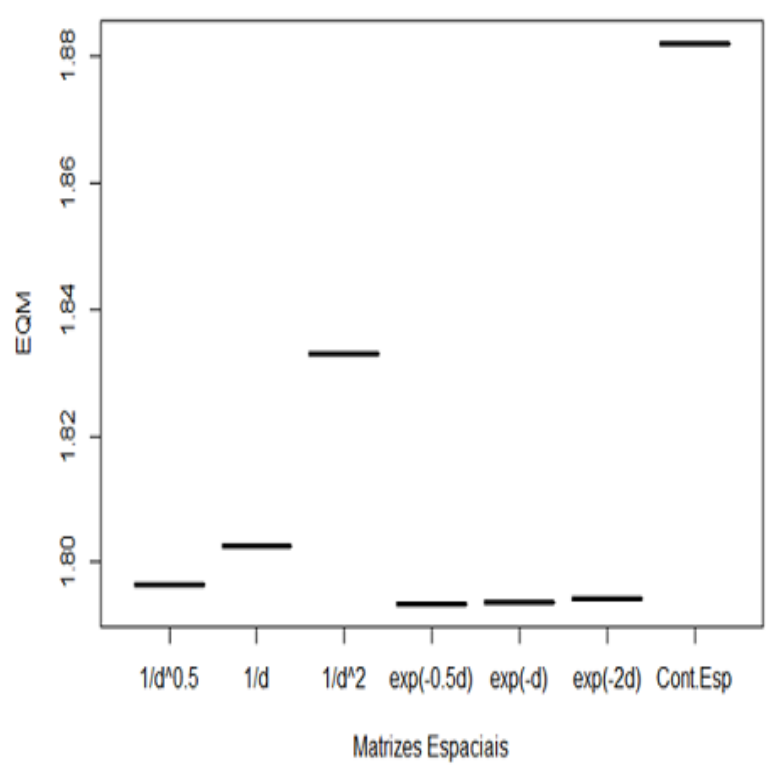

10 Regiöes

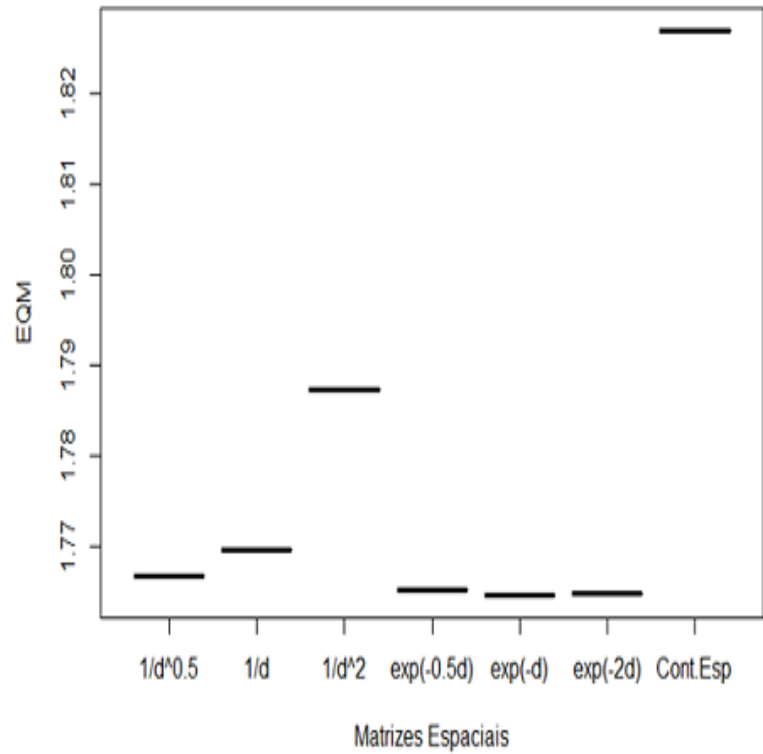

Figura 4.10: Simulação das séries pelo Gneiting $\mu=10$ e $\beta=0,9$; EQM calculados ajustando os modelos $\operatorname{STAR}\left(1_{1}\right)$ com as matrizes de pesos espaciais $\frac{1}{d^{0,5}}, \frac{1}{d}, \frac{1}{d^{2}}, e^{-0,5 d}$, $e^{-d}, e^{-2 d}$ e contiguidade espacial de ordem 1 para 5 e 10 regiões.

5 Regiões

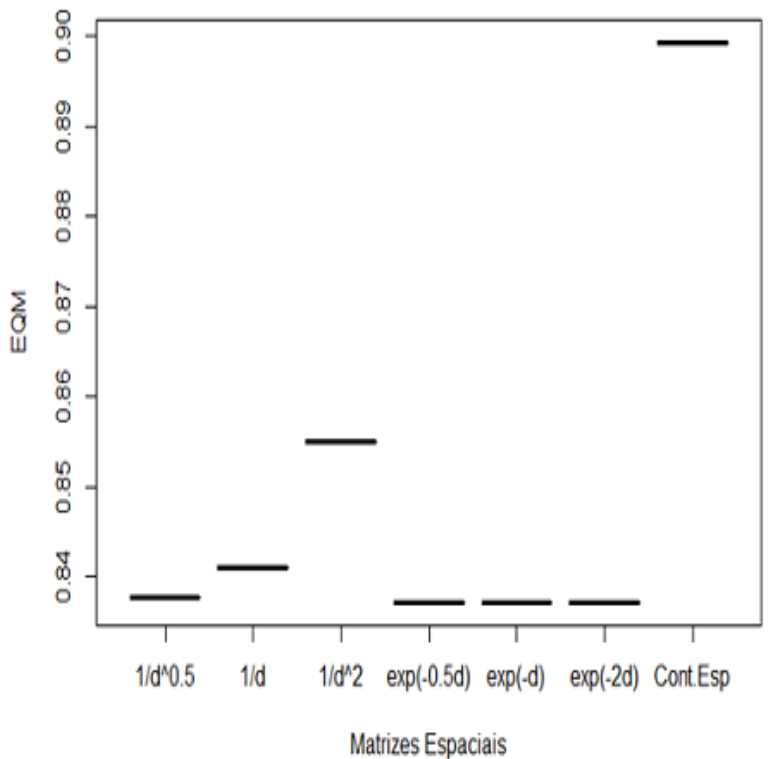

10 Regiöes

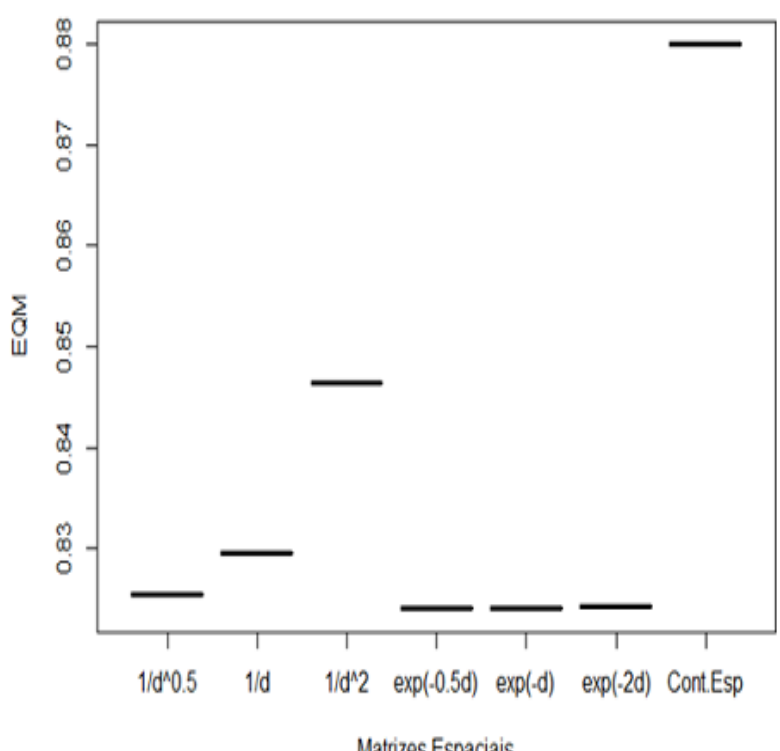

Matrizes Espaciais

Figura 4.11: Simulação das séries pelo Gneiting $\mu=2$ e $\beta=0,9$; EQM calculados ajustando os modelos $\operatorname{STAR}\left(1_{1}\right)$ com as matrizes de pesos espaciais $\frac{1}{d^{0,5}}, \frac{1}{d}, \frac{1}{d^{2}}, e^{-0,5 d}$, $e^{-d}, e^{-2 d}$ e contiguidade espacial de ordem 1 para 5 e 10 regiões. 

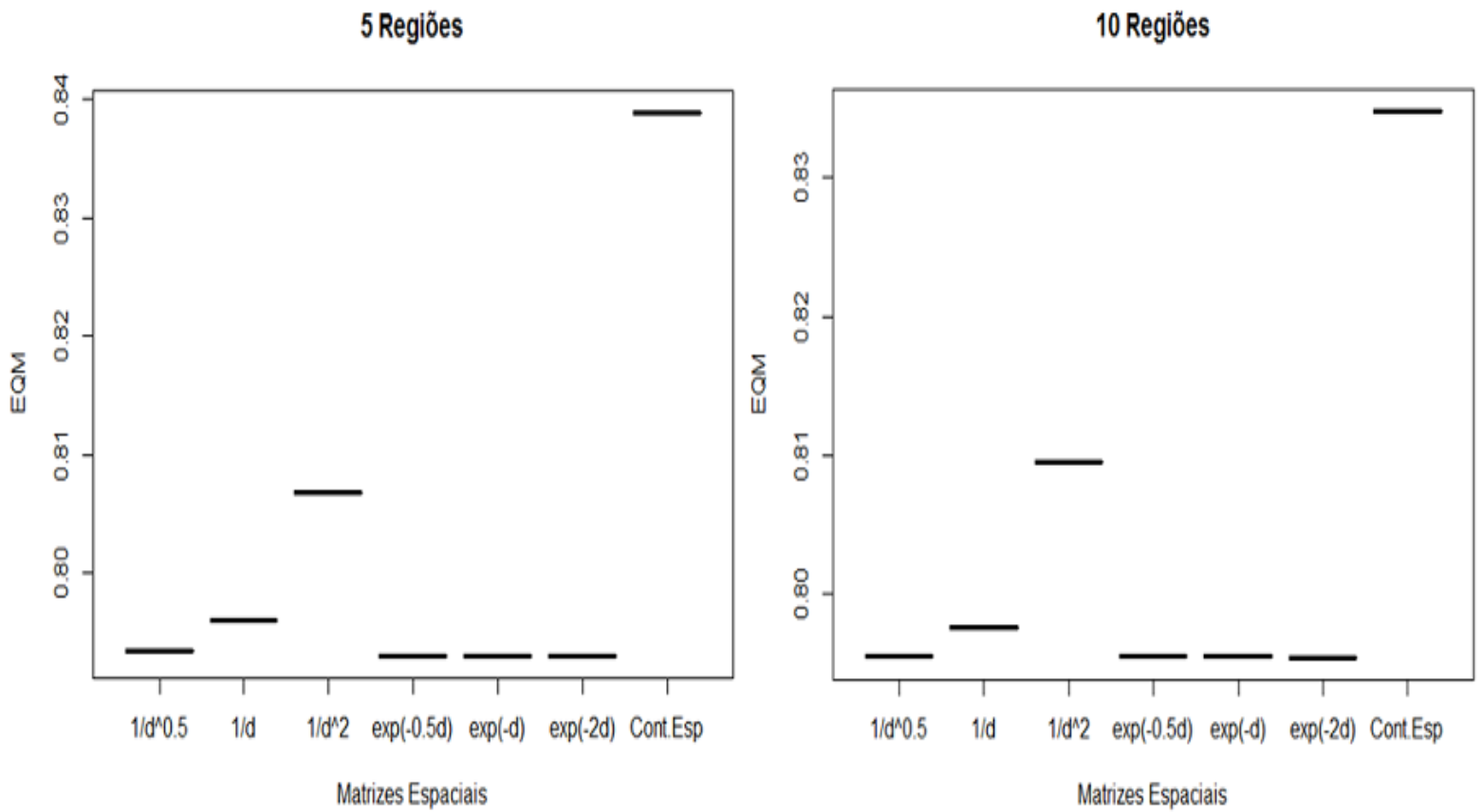

Figura 4.12: Simulação das séries pelo Gneiting $\mu=1,11$ e $\beta=0,9$; EQM calculados ajustando os modelos $\operatorname{STAR}\left(1_{1}\right)$ com as matrizes de pesos espaciais $\frac{1}{d^{0,5}}, \frac{1}{d}, \frac{1}{d^{2}}, e^{-0,5 d}$, $e^{-d}, e^{-2 d}$ e contiguidade espacial de ordem 1 para 5 e 10 regiões.

\begin{tabular}{cccccccc}
\hline & \multicolumn{7}{c}{ Matriz de Peso Espacial } \\
\cline { 2 - 8 } Modelo Simulado & $\frac{1}{d^{0,5}}$ & $\frac{1}{d}$ & $\frac{1}{d^{2}}$ & $e^{-0,5 d}$ & $e^{-d}$ & $e^{-2 d}$ & Cont.Esp \\
\hline$\mu=10, \beta=0$ & 1,7552 & 1,7588 & 1,7744 & 1,7545 & $\mathbf{1 , 7 5 4 4}$ & $\mathbf{1 , 7 5 4 4}$ & 1,8257 \\
$\mu=2, \beta=0$ & 0,8267 & 0,8303 & 0,8451 & $\mathbf{0 , 8 2 5 9}$ & $\mathbf{0 , 8 2 5 9}$ & $\mathbf{0 , 8 2 5 9}$ & 0.8915 \\
$\mu=1,11, \beta=0$ & 0,8157 & 0,8187 & 0,8302 & $\mathbf{0 , 8 1 4 9}$ & $\mathbf{0 , 8 1 4 9}$ & 0,8150 & 0,8621 \\
$\mu=10, \beta=0,5$ & 1,8034 & 1,8071 & 1,8229 & 1,8027 & $\mathbf{1 , 8 0 2 6}$ & 1,8027 & 1,8745 \\
$\mu=2, \beta=0,5$ & 0,8357 & 0,8404 & 0,8565 & $\mathbf{0 , 8 3 3 9}$ & 0,8340 & 0,8343 & 0,9035 \\
$\mu=1,11, \beta=0,5$ & 0,8007 & 0,8040 & 0,8155 & $\mathbf{0 , 7 9 9 7}$ & 0,7998 & 0,7999 & 0,8452 \\
$\mu=10, \beta=0,9$ & 1,7963 & 1,8026 & 1,8331 & $\mathbf{1 , 7 9 3 4}$ & 1,7937 & 1,7942 & 1,8820 \\
$\mu=2, \beta=0,9$ & 0,8377 & 0,8409 & 0,8549 & $\mathbf{0 , 8 3 7 2}$ & $\mathbf{0 , 8 3 7 2}$ & $\mathbf{0 , 8 3 7 2}$ & 0,8992 \\
$\mu=1,11, \beta=0,9$ & 0,7934 & 0,7960 & 0,8067 & $\mathbf{0 , 7 9 3 0}$ & $\mathbf{0 , 7 9 3 0}$ & $\mathbf{0 , 7 9 3 0}$ & 0,8389 \\
\hline Média & 1,1405 & 1,1443 & 1,1599 & $\mathbf{1 , 1 3 9 5}$ & $\mathbf{1 , 1 3 9 5}$ & 1,1396 & 1,2025 \\
\hline
\end{tabular}

Tabela 4.1: EQM calculados pelos modelos ajustados de $\operatorname{STAR}\left(1_{1}\right)$ com as matrizes de pesos espaciais $\frac{1}{d^{0,5}}, \frac{1}{d}, \frac{1}{d^{2}}, e^{-0,5 d}, e^{-d}, e^{-2 d}$ e contiguidade espacial (Cont.Esp) de ordem 1 para 5 regiões, simulando os modelos de Gneiting 


\begin{tabular}{cccccccc}
\hline & \multicolumn{7}{c}{ Matriz de Peso Espacial } \\
\cline { 2 - 8 } Modelo Simulado & $\frac{1}{d^{0,5}}$ & $\frac{1}{d}$ & $\frac{1}{d^{2}}$ & $e^{-0,5 d}$ & $e^{-d}$ & $e^{-2 d}$ & Cont.Esp \\
\hline$\mu=10, \beta=0$ & 1,7683 & 1,7793 & 1,7931 & 1,7674 & $\mathbf{1 , 7 6 7 2}$ & 1,7680 & 1,8213 \\
$\mu=2, \beta=0$ & 0,8204 & 0,8232 & 0,8376 & $\mathbf{0 , 8 2 0 0}$ & $\mathbf{0 , 8 2 0 0}$ & $\mathbf{0 , 8 2 0 0}$ & 0,8682 \\
$\mu=1,11, \beta=0$ & 0,8002 & 0,8025 & 0,8145 & $\mathbf{0 , 7 9 9 8}$ & $\mathbf{0 , 7 9 9 8}$ & $\mathbf{0 , 7 9 9 8}$ & 0,8363 \\
$\mu=10, \beta=0,5$ & 1,8237 & 1,8614 & 1,8351 & $\mathbf{1 , 8 1 2 2}$ & 1,8127 & 1,8128 & 1,8786 \\
$\mu=2, \beta=0,5$ & 0,8322 & 0,8347 & 0,8483 & $\mathbf{0 , 8 3 2 0}$ & $\mathbf{0 , 8 3 2 0}$ & $\mathbf{0 , 8 3 2 0}$ & 0,8766 \\
$\mu=1,11, \beta=0,5$ & 0,7722 & 0,7748 & 0,7875 & $\mathbf{0 , 7 7 1 7}$ & $\mathbf{0 , 7 7 1 7}$ & 0,7718 & 0,8118 \\
$\mu=10, \beta=0,9$ & 1,7668 & 1,7696 & 1,7874 & 1,7652 & $\mathbf{1 , 7 6 4 7}$ & 1,7649 & 1,8269 \\
$\mu=2, \beta=0,9$ & 0,8254 & 0,8295 & 0,8465 & $\mathbf{0 , 8 2 4 0}$ & 0,8241 & 0,8243 & 0,8800 \\
$\mu=1,11, \beta=0,9$ & $\mathbf{0 , 7 9 5 5}$ & 0,7976 & 0,8095 & $\mathbf{0 , 7 9 5 5}$ & $\mathbf{0 , 7 9 5 5}$ & 0,7954 & 0,8348 \\
\hline Média & 1,1339 & 1,1414 & 1,1510 & $\mathbf{1 , 1 3 2 0}$ & $\mathbf{1 , 1 3 2 0}$ & 1,1321 & 1,1816 \\
\hline
\end{tabular}

Tabela 4.2: EQM calculados pelos modelos ajustados de $\operatorname{STAR}\left(1_{1}\right)$ com as matrizes de pesos espaciais $\frac{1}{d^{0,5}}, \frac{1}{d}, \frac{1}{d^{2}}, e^{-0,5 d}, e^{-d}, e^{-2 d}$ e contiguidade espacial (Cont.Esp) de ordem 1 para 10 regiões, simulando os modelos de Gneiting

\begin{tabular}{cccccccccc}
\hline & \multicolumn{7}{c}{ Tempo de Execução (em segundo) } \\
\cline { 2 - 9 } Modelo Simulado & Gerar Séries & $\frac{1}{d^{0,5}}$ & $\frac{1}{d}$ & $\frac{1}{d^{2}}$ & $e^{-0,5 d}$ & $e^{-d}$ & $e^{-2 d}$ & Cont.Esp & Total.Simulação \\
\hline$\mu=10, \beta=0$ & 10,152 & 0,081 & 0,065 & 0,054 & 0,064 & 0,061 & 0,057 & 0,063 & 10,597 \\
$\mu=2, \beta=0$ & 7,722 & 0,073 & 0,052 & 0,068 & 0,062 & 0,053 & 0,064 & 0,064 & 8,158 \\
$\mu=1,11, \beta=0$ & 11,030 & 0,094 & 0,052 & 0,069 & 0,051 & 0,057 & 0,061 & 0,051 & 11,465 \\
$\mu=10, \beta=0,5$ & 14,773 & 0,105 & 0,061 & 0,064 & 0,053 & 0,058 & 0,062 & 0,055 & 15,231 \\
$\mu=2, \beta=0,5$ & 14,802 & 0,087 & 0,061 & 0,061 & 0,066 & 0,060 & 0,062 & 0,056 & 15,255 \\
$\mu=1,11, \beta=0,5$ & 15,012 & 0,074 & 0,052 & 0,067 & 0,058 & 0,055 & 0,060 & 0,054 & 15,432 \\
$\mu=10, \beta=0,9$ & 12,196 & 0,075 & 0,060 & 0,062 & 0,059 & 0,063 & 0,059 & 0,057 & 12,631 \\
$\mu=2, \beta=0,9$ & 12,476 & 0,096 & 0,061 & 0,061 & 0,055 & 0,062 & 0,057 & 0,057 & 12,925 \\
$\mu=1,11, \beta=0,9$ & 12,409 & 0,082 & 0,061 & 0,068 & 0,053 & 0,065 & 0,063 & 0,054 & 12,855 \\
\hline Média & 12,286 & 0,085 & 0,058 & 0,064 & 0,058 & 0,059 & 0,061 & 0,057 & 12,728 \\
\hline
\end{tabular}

Tabela 4.3: Tempos decorridos para geração de 5 séries e estimação dos modelos de STAR com as matrizes de pesos espaciais $\frac{1}{d^{0,5}}, \frac{1}{d}, \frac{1}{d^{2}}, e^{-0,5 d}, e^{-d}, e^{-2 d}$ e contiguidade espacial (Cont.Esp) de ordem 1, simulando os modelos de Gneiting 


\begin{tabular}{cccccccccc}
\hline & \multicolumn{7}{c}{ Tempo de Execução (em segundo) } \\
\cline { 2 - 9 } Modelo Simulado & Gerar Séries & $\frac{1}{d^{0,5}}$ & $\frac{1}{d}$ & $\frac{1}{d^{2}}$ & $e^{-0,5 d}$ & $e^{-d}$ & $e^{-2 d}$ & Cont.Esp & Total.Simulação \\
\hline$\mu=10, \beta=0$ & 48,420 & 0,107 & 0,093 & 0,092 & 0,093 & 0,092 & 0,092 & 0,092 & 49,081 \\
$\mu=2, \beta=0$ & 49,605 & 0,109 & 0,092 & 0,090 & 0,095 & 0,095 & 0,093 & 0,092 & 50,271 \\
$\mu=1,11, \beta=0$ & 49,932 & 0,114 & 0,091 & 0,093 & 0,095 & 0,097 & 0,096 & 0,097 & 50,615 \\
$\mu=10, \beta=0,5$ & 88,848 & 0,106 & 0,097 & 0,096 & 0,096 & 0,091 & 0,091 & 0,089 & 89,514 \\
$\mu=2, \beta=0,5$ & 87,892 & 0,105 & 0,098 & 0,092 & 0,092 & 0,091 & 0,092 & 0,089 & 88,551 \\
$\mu=1,11, \beta=0,5$ & 88,926 & 0,108 & 0,097 & 0,089 & 0,091 & 0,091 & 0,090 & 0,090 & 89,582 \\
$\mu=10, \beta=0,9$ & 55,313 & 0,122 & 0,090 & 0,088 & 0,090 & 0,087 & 0,089 & 0,094 & 55,973 \\
$\mu=2, \beta=0,9$ & 55,529 & 0,295 & 0,093 & 0,090 & 0,088 & 0,092 & 0,090 & 0,085 & 56,362 \\
$\mu=1,11, \beta=0,9$ & 56,372 & 0,112 & 0,092 & 0,093 & 0,095 & 0,093 & 0,090 & 0,090 & 57,037 \\
\hline Média & 64,537 & 0,131 & 0,094 & 0,091 & 0,093 & 0,092 & 0,091 & 0,091 & 65,221 \\
\hline
\end{tabular}

Tabela 4.4: Tempos decorridos para geração de 10 séries e estimação dos modelos de STAR com as matrizes de pesos espaciais $\frac{1}{d^{0,5}}, \frac{1}{d}, \frac{1}{d^{2}}, e^{-0,5 d}, e^{-d}, e^{-2 d}$ e contiguidade espacial (Cont.Esp) de ordem 1, simulando os modelos de Gneiting 


\section{Capítulo 5}

\section{Aplicação}

Os modelos STARMA começaram a ser bastante aplicados nos últimos anos na modelagem de dados climatológicos e econômicos. Por exemplo, Rao e Antunes (2004) usaram a classe de modelo STARMA para ajustar as séries de temperaturas mensais de 9 regiões do Reino Unido. Zheng et al. (2014) ajustaram os dados de quantidade de precipitação por 5, 10 e 15 minutos utilizando os modelos de STARMA para analisar e prever as possíveis enchentes de vários locais da cidade Pequim nos dias de tempestade, e mostrou um bom desempenho de ajuste da classe de modelos STARMA. Zhou e Buongiorno (2006) desenvolveram um modelo econométrico espaço-tempo usando modelo STARMA para os preços trimestrais da madeira de serraria de pinho de 21 regiões geograficamente contíguas no sul dos Estados Unidos e mostrou bom resultado de ajuste.

\section{1 Índice de Preço Imobiliário de Venda}

Neste capítulo, aplicamos a classe de modelos STARMA para analisar as séries do índice fipezap de preço imobiliário de venda para as seis cidades metropolitanas de São Paulo. Para a parte estimação de modelos, utilizamos as matrizes de pesos espaciais do tipo exponencial $e^{-0,5 d}, e^{-d}$ e $e^{-2 d}$, que mostraram os melhores ajustes de modelos nas simulações. E com a ajuda do pactoe starma no $R$, o Filtro de Kalman é utilizado para a estimação dos modelos STARMA.

\subsubsection{Descrição de Dados}

Utilizamos dados mensais do índice fipezap de preço imobiliário de venda para apartamentos de 2 dormitórios das seis cidades metropolitanas de São Paulo, o período de análise é de Janeiro de 2013 a Fevereiro de 2017. Os dados são disponíveis pelo site de fipezap: http://fipezap.zapimoveis.com.br.

O índice fipezap de preço imobiliário de venda, que também pode ser interpretado como um tipo de preço imobiliário relativo, é o primeiro indicador com abrangência nacional que acompanha os preços de venda de imóveis no Brasil. O índice é calculado 
pela FIPE com base nos anúncios de apartamentos prontos publicados na página do ZAP Imóveis e em outras fontes da Internet.

As seis cidades no estudo são: Santo André, São Bernardo do Campo, São Caetano do Sul, São Paulo, Guarulhos e Osasco. As coordenadas geográficas e as localizações das cidades estão apresentadas na Tabela 5.1 e na Figura 5.1.

\begin{tabular}{ccc}
\hline Cidade & Latitude & Longitude \\
\hline Santo André & $-23,66$ & $-46,53$ \\
São Bernardo do Campo & $-23,69$ & $-46,56$ \\
São Caetano do Sul & $-23,62$ & $-46,55$ \\
São Paulo & $-23,54$ & $-46,63$ \\
Guarulhos & $-23,46$ & $-46,53$ \\
Osasco & $-23,53$ & $-46,78$ \\
\hline
\end{tabular}

Tabela 5.1: As coordenadas geográficas das cidades do estudo

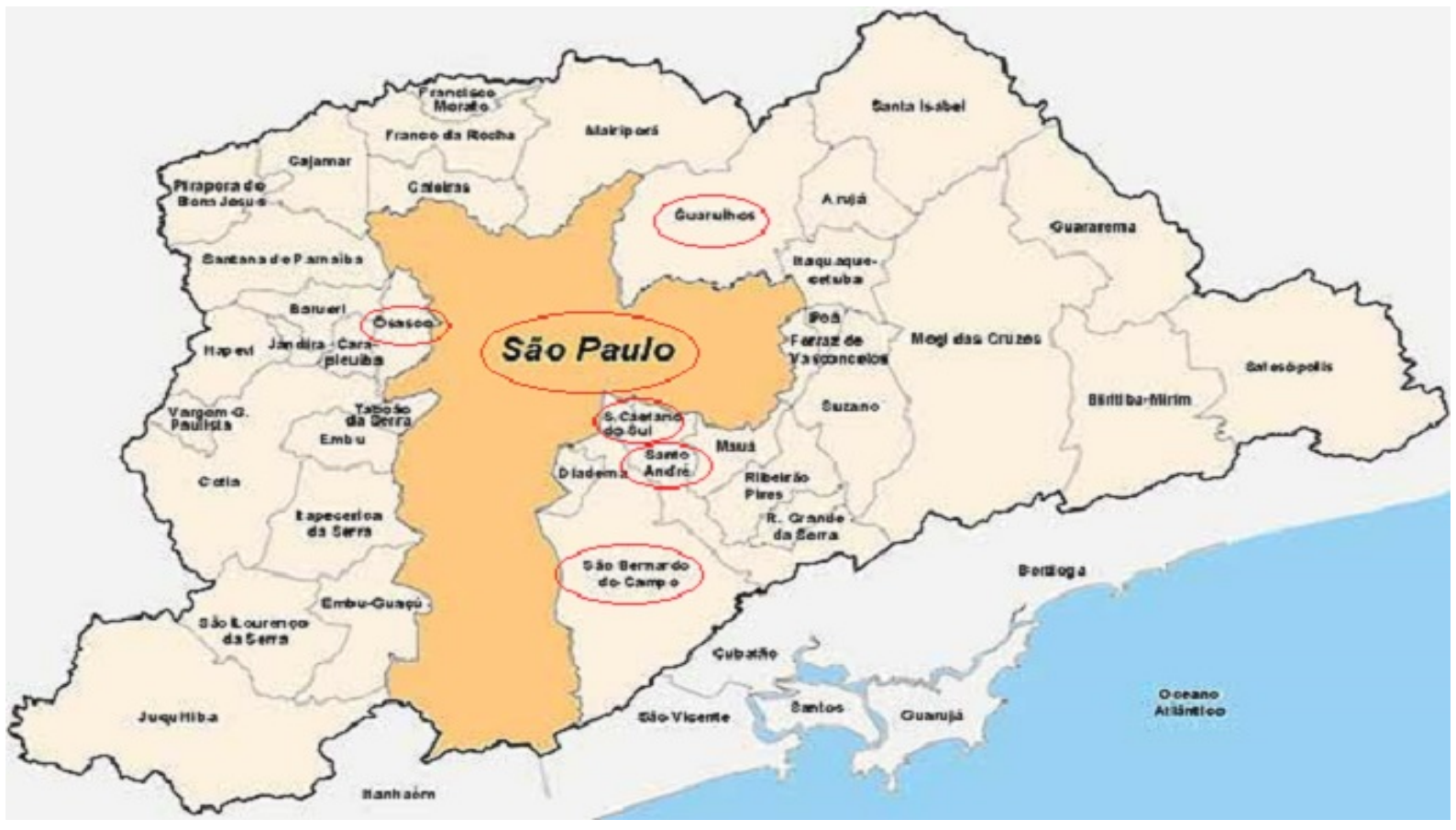

Figura 5.1: As localizações das cidades do estudo 


\subsubsection{Análise Descritiva}

Analisamos descritivamente as séries do índice de preço imobiliário de venda, pela Figura 5.2 percebemos que todas as séries possuem uma tendência crescente. Foram feitos testes de raíz unitária de Philippe Perron (Morettin (2008)), que podem ser encontrados na Tabela 5.2; pelos p valores do teste não rejeitamos a hipótese nula de que todas as séries do índice de preço têm raíz unitária (tendência). Nesse caso, antes de aplicar modelos STARMA, tiramos uma diferença das séries para elas tornarem estacionárias.
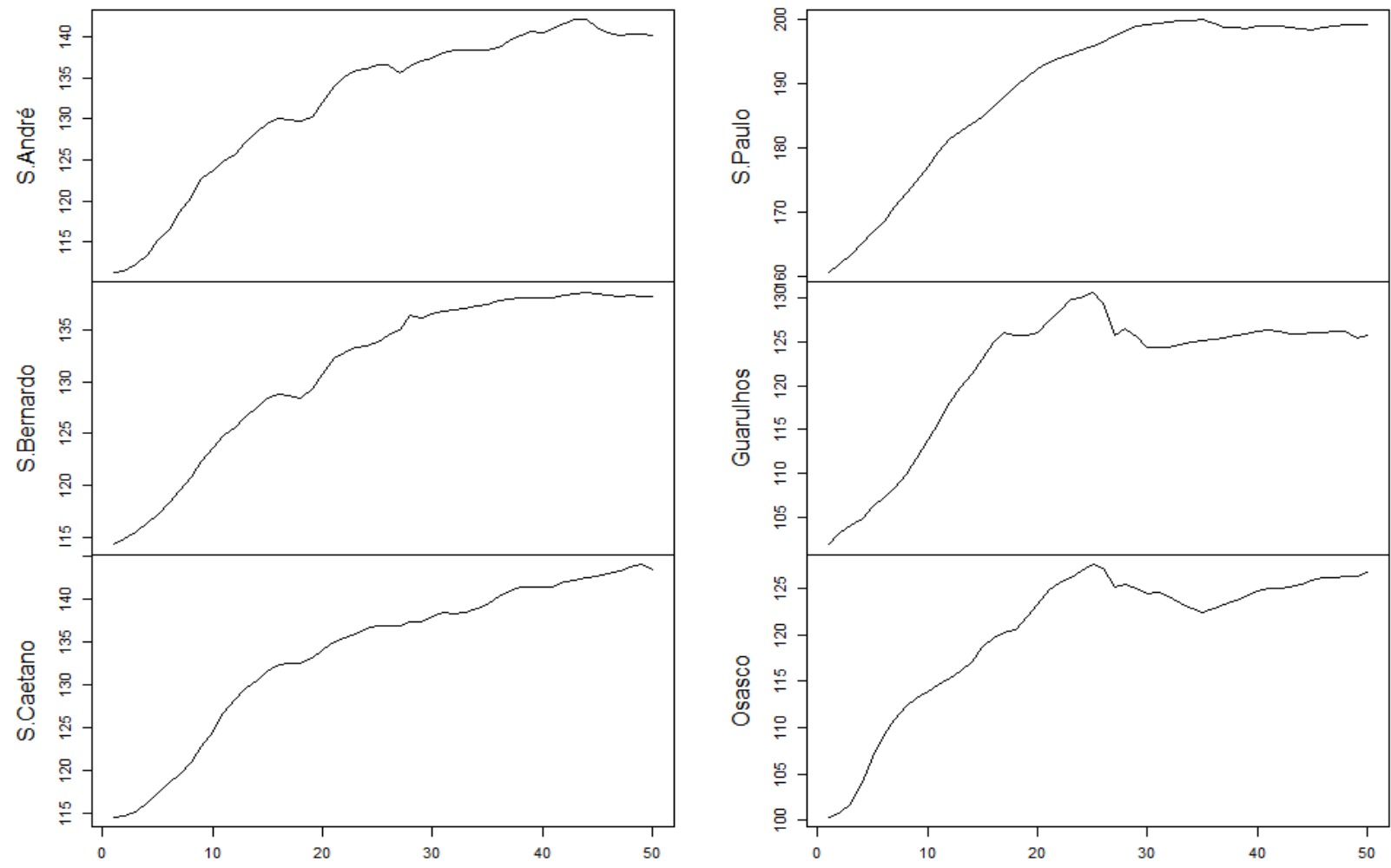

Figura 5.2: As séries do índice fipezap de preço imobiliário de venda

\begin{tabular}{cc}
\hline Cidade & P valor \\
\hline Santo André & 0,9614 \\
São Bernardo do Campo & 0,9866 \\
São Caetano do Sul & 0,9230 \\
São Paulo & 0,8941 \\
Guarulhos & 0,7527 \\
Osasco & 0,4574 \\
\hline
\end{tabular}

Tabela 5.2: Teste raíz unitária de Philippe Perron 


\subsubsection{Identificação, Estimação, Diagnóstco e Previsão}

Construímos a matriz de pesos espaciais $\mathbf{W}$, dada a seguir, utilizando a exponencial da distância geodésica, $e^{-0,5 d}$.

$$
\boldsymbol{W}^{\mathbf{1}}=\left[\begin{array}{ccccccc} 
& \mathrm{r} 1 & \mathrm{r} 2 & \mathrm{r} 3 & \mathrm{r} 4 & \mathrm{r} 5 & \mathrm{r} 6 \\
\mathrm{r} 1 & 0 & 0,2011 & 0,2010 & 0,1998 & 0,1993 & 0,1988 \\
\mathrm{r} 2 & 0,2012 & 0 & 0,2009 & 0,1999 & 0,1991 & 0,1989 \\
\mathrm{r} 3 & 0,2009 & 0,2006 & 0 & 0,2002 & 0,1996 & 0,1987 \\
\mathrm{r} 4 & 0,1998 & 0,1997 & 0,2003 & 0 & 0,2002 & 0,2000 \\
\mathrm{r} 5 & 0,1999 & 0,1996 & 0,2003 & 0,2007 & 0 & 0,1995 \\
\mathrm{r} 6 & 0,1995 & 0,1997 & 0,2000 & 0,2009 & 0,1999 & 0
\end{array}\right]
$$

Lembrando que a soma de cada linha da matriz é um, e o peso $w_{i j}$ fica maior quando i e j são vizinhos próximos. As siglas r1, r2, r3, r4, r5 e r6 representam respectivamente a cidade Santo André, São Bernardo do Campo, São Caetano do Sul, São Paulo, Guarulhos e Osasco.

Para as séries diferenciadas sem tendência, obtemos os gráficos de autocorrelação espaço-temporal STFAC e autocorrelação parcial espaço-temporal STFACP em relação aos lags (defasagens) de tempo, apresentados nas Figuras 5.3 e 5.4. Pelo gráfico de STFAC, vemos que as autocorrelações de espaço-temporais decaem exponencialmente, isso é um indício de existir um componente autorregressivo. Pelo gráfico de STFACP, observamos que apenas o lag 1 das autocorrelações dos próprios locais (vizinhança ordem 0) é significativa; e os lags 1, 2 e 3 das autocorrelações espaço-temporais de vizinhança ordem 1 são significativas que estão fora do intervalo de confiança. Portanto testamos primeiro o modelo STAR $\left(1_{1}\right)$, os parâmetros $\phi_{10}$ e $\phi_{11}$ foram significativos, com o EQM ajuste do modelo 0,284. No modelo $\operatorname{STAR}\left(1_{1}\right)$, ajustamos um lag 2 para os vizinhos, estimamos o modelo $\operatorname{STAR}\left(2_{1}\right)$, porém o parâmetro $\phi_{11}$ não foi significativo com $\mathrm{p}$ valor 0,5498, e o modelo final STAR $\left(2_{1}\right)$ está na Tabela 5.3 com o EQM de ajuste 0,279 . Pelo EQM, a medida de ajuste dos modelos, escolhemos o $\operatorname{STAR}\left(2_{1}\right)$ como o modelo final por ter o menor EQM, e o modelo pode ser escrito também da seguinte forma:

$$
\boldsymbol{Z}_{t}=0,6221 \boldsymbol{I}_{\mathbf{6}} \boldsymbol{Z}_{t-1}+0,2904 \boldsymbol{W}^{\mathbf{1}} \boldsymbol{Z}_{t-2}+\boldsymbol{\epsilon}_{t}
$$

em que $\boldsymbol{Z}_{t}$ as séries do índice imobiliário das seis cidades, $\boldsymbol{W}^{\mathbf{1}}$ a matriz espacial e $\boldsymbol{\epsilon}_{t}$ os erros aleatórios. 
Foram feitos os gráficos de STFAC e STFACP dos resíduos do modelo $\operatorname{STAR}\left(2_{1}\right)$ nas Figuras 5.5 e 5.6. Percebemos que com o modelo $\operatorname{STAR}\left(2_{1}\right)$ conseguiu capturar as interdependências espaciais por não ter lag relevantemente significativo fora do intervalo de confiança das STFAC e STFACP.

\begin{tabular}{cccc}
\hline Parâmetro & Estimativa & Erro Padrão & P Valor \\
\hline$\phi_{10}$ & 0,6221 & 0,0554 & $<2,2 \times 10^{-16}$ \\
$\phi_{21}$ & 0,2904 & 0,0622 & $4,672 \times 10^{-6}$ \\
\hline
\end{tabular}

Tabela 5.3: Os parâmetros estimados do modelo $\operatorname{STAR}\left(2_{1}\right)$ com a matriz de pesos espaciais $e^{-0,5 d}$

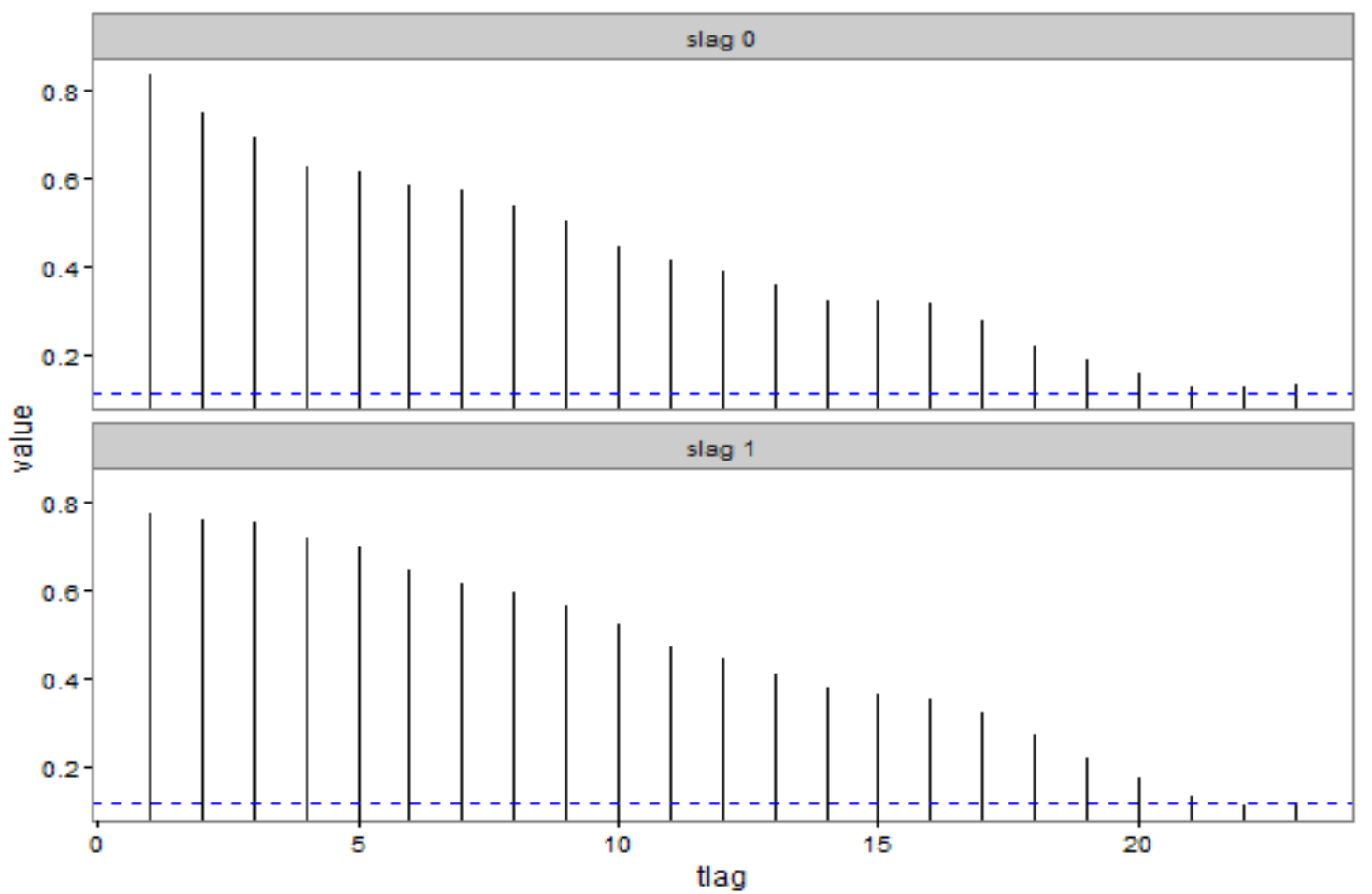

Figura 5.3: STFAC das séries do índice de preço imobiliário de venda diferenciadas 


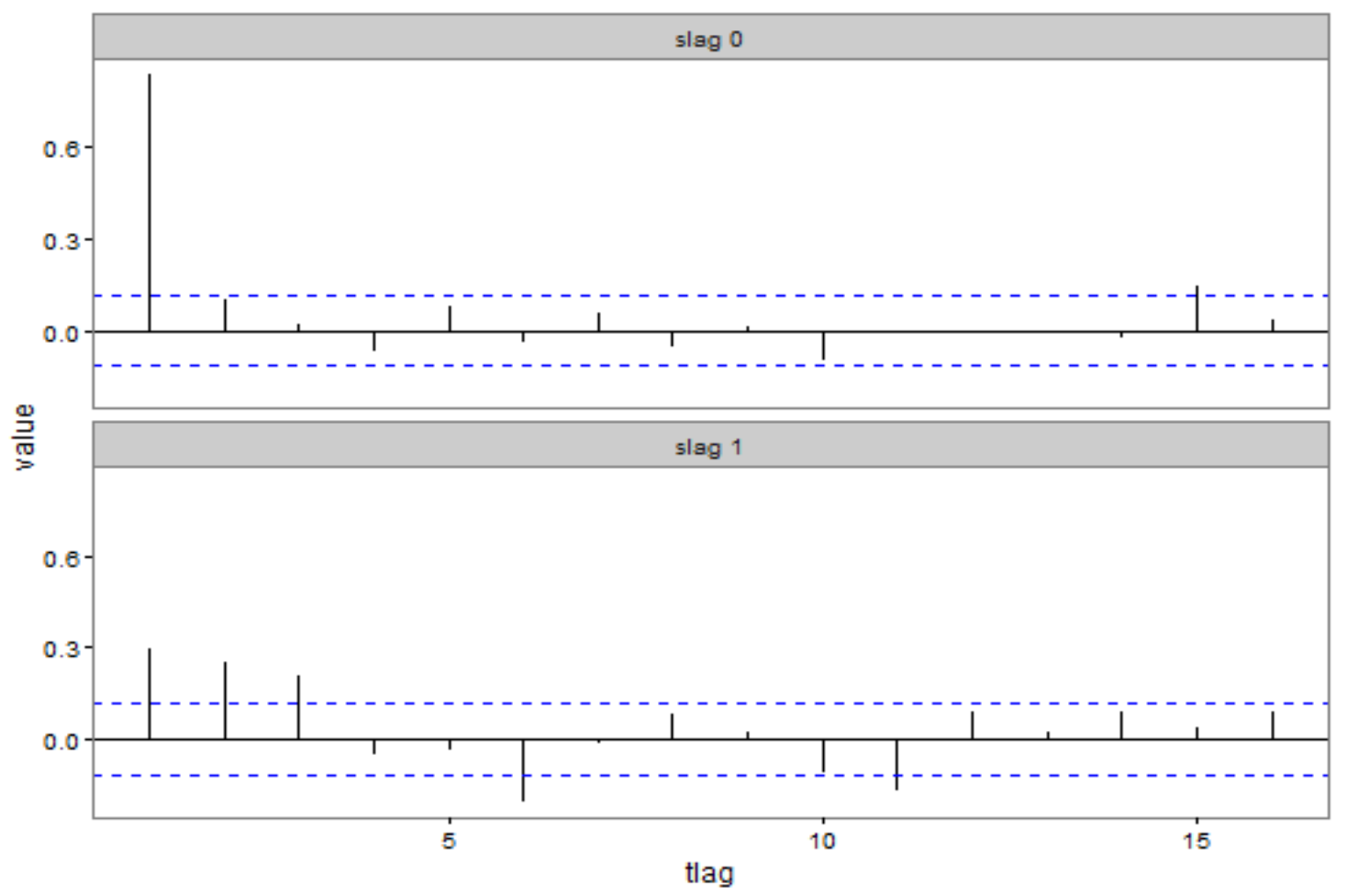

Figura 5.4: STFACP das séries do índice de preço imobiliário de venda diferenciadas

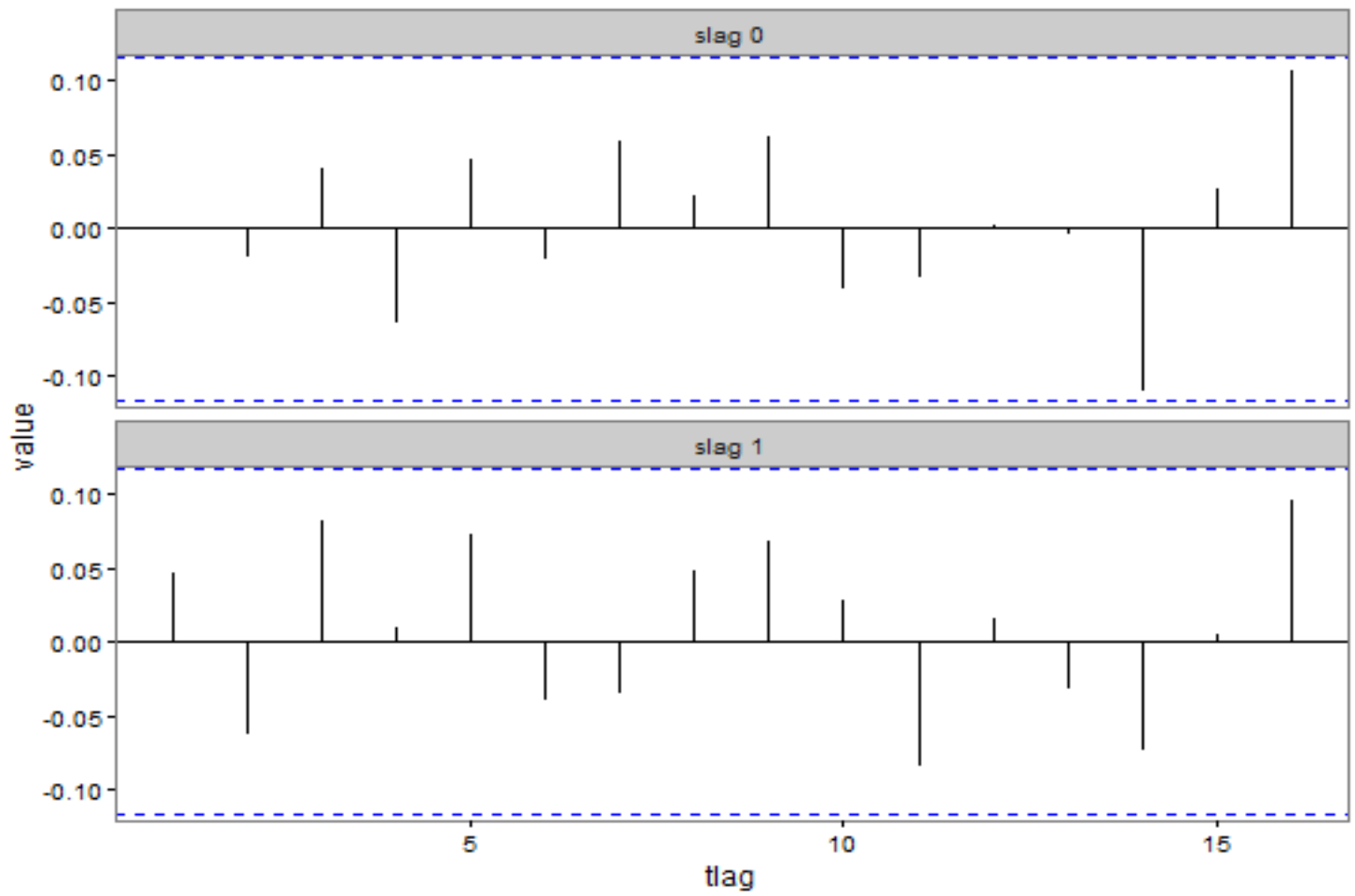

Figura 5.5: STFAC dos resíduos do modelo final $\operatorname{STAR}\left(2_{1}\right)$ 


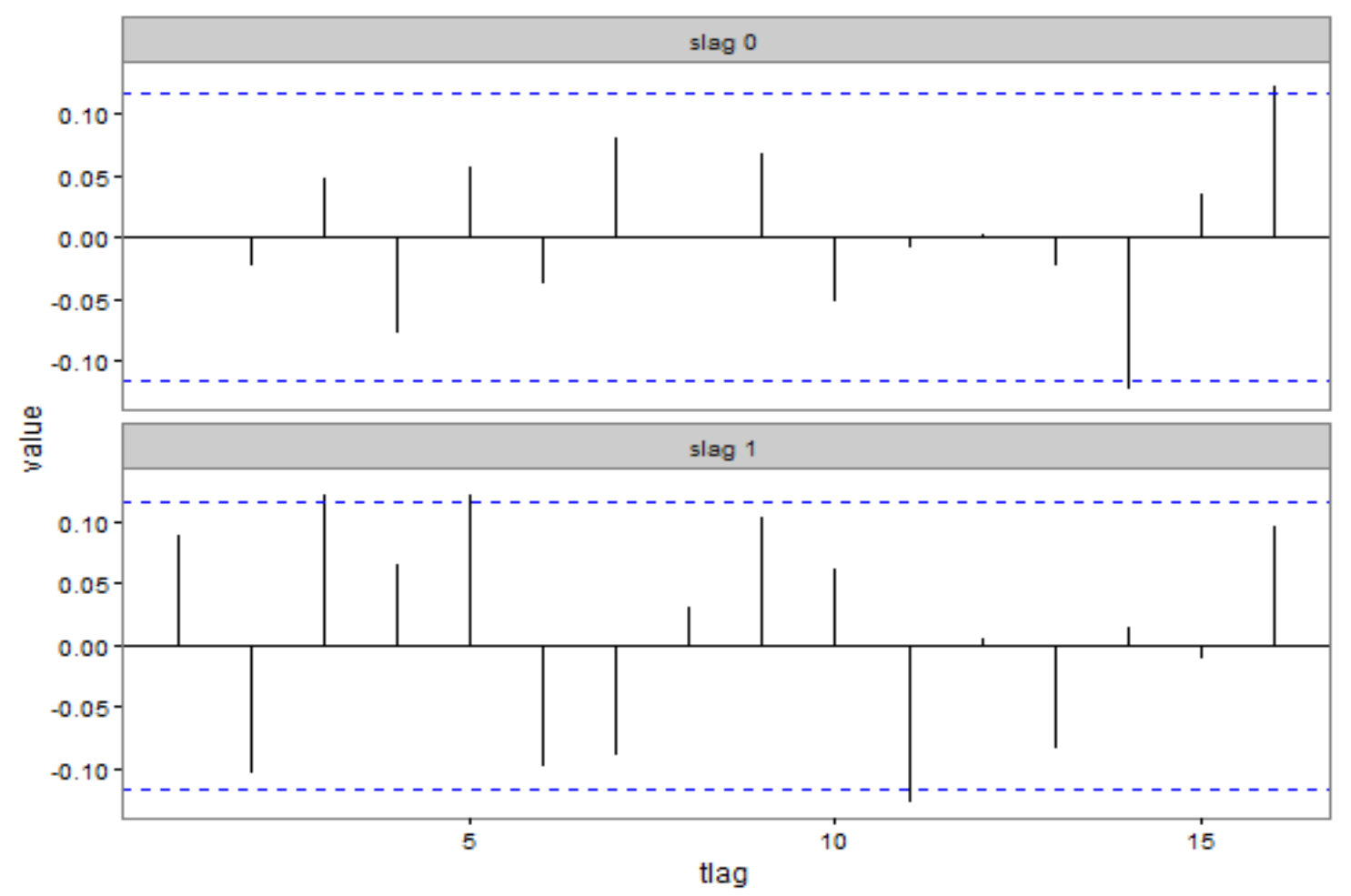

Figura 5.6: STFACP dos resíduos do modelo final $\operatorname{STAR}\left(2_{1}\right)$

Agora ajustamos o modelo ARMA univariado para cada série diferenciada do índice de preços imobiliários de venda. A qualidade de ajuste EQM e a melhor ordem de autorregressivo e de média móvel estão apresentados na Tabela 5.4, e a qualidade de ajuste do modelo $\operatorname{STAR}\left(2_{1}\right)$ para cada cidade na Tabela 5.5.

\begin{tabular}{cccc}
\hline Cidade & Termo autorregressivo & Termo Médias Móveis & EQM \\
\hline Santo André & 1 & 1 & 0,296 \\
São Bernardo do Campo & 1 & 1 & 0,148 \\
São Caetano do Sul & 0 & 3 & 0,142 \\
São Paulo & 1 & 0 & 0,074 \\
Guarulhos & 0 & 3 & 0,639 \\
Osasco & 0 & 2 & 0,442 \\
\hline Geral & & 0,290 \\
\hline
\end{tabular}

Tabela 5.4: A qualidade de ajuste e as ordens de autorregressivo e de média móvel do modelo ARMA univariada para cada cidade do estudo

Comparando a Tabela 5.4 e 5.5, vemos que o modelo STAR ajusta melhor do que modelos univariados ARMA por ter mais cidades com EQM menores. O EQM geral dos modelos univariados ARMA $(0,290)$ é maior do que o $\operatorname{EQM~do~modelo~} \operatorname{STAR}\left(2_{1}\right)$ que é 0,279 . Isso mostrou a eficácia de ajuste da classe de modelos STARMA para as séries temporais espacialmente localizadas. Além disso, a modelagem de modelos STARMA custa um tempo menor do que modelos univariados ARMA, principalmente quando estudo 


\begin{tabular}{cc}
\hline Cidade & EQM \\
\hline Santo André & 0,287 \\
São Bernardo do Campo & 0,136 \\
São Caetano do Sul & 0,110 \\
São Paulo & 0,139 \\
Guarulhos & 0,653 \\
Osasco & 0,352 \\
\hline Geral & 0,279 \\
\hline
\end{tabular}

Tabela 5.5: A qualidade de ajuste do modelo $\operatorname{STAR}\left(2_{1}\right)$ para cada cidade do estudo

envolve uma grande quantidade de regiões; pois a modelagem de modelos univariados exigem aplicação em cada série.

Por fim, na Figura 5.7 e na Tabela 5.6 encontram-se as previsões de 5 passos a frente referentes aos meses de Março, Abril, Maio, Junho e Julho do ano 2017.

\begin{tabular}{cccccc}
\hline Cidade & Março 2017 & Abril 2017 & Maio 2017 & Junho 2017 & Julho 2017 \\
\hline Santo André & 140,13 & 140,06 & 140,02 & 139,98 & 139,97 \\
São Bernardo do Campo & 138,25 & 138,26 & 138,25 & 138,24 & 138,22 \\
São Caetano do Sul & 143,05 & 142,85 & 142,74 & 142,68 & 142,64 \\
São Paulo & 199,03 & 199,02 & 199,00 & 198,99 & 198,97 \\
Guarulhos & 125,82 & 125,89 & 125,92 & 125,93 & 125,92 \\
Osasco & 126,85 & 126,93 & 126,96 & 126,97 & 126,96 \\
\hline
\end{tabular}

Tabela 5.6: As previsões de 5 passos para frente usando o modelo $\operatorname{STAR}\left(2_{1}\right)$ 

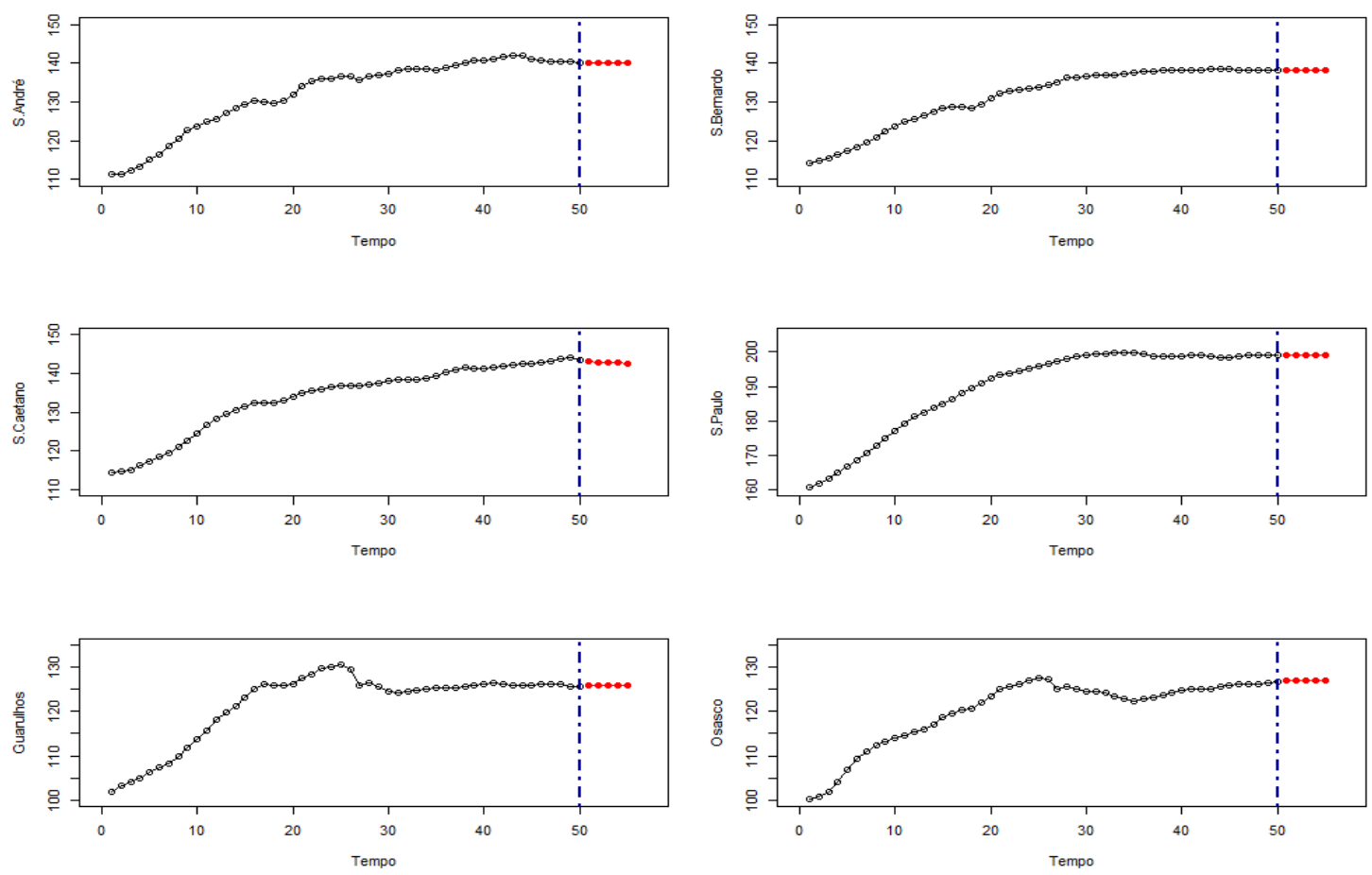

Figura 5.7: As previsões de 5 passos a frente usando o modelo $\operatorname{STAR}\left(2_{1}\right)$

Testamos outros tipos de pesos espaciais exponenciais para ilustrar a concordância dos resultados obtidos na aplicação com os da simulação.

Utilizamos peso espacial $e^{-d}$ e foi obtido o melhor modelo $\operatorname{STAR}\left(2_{1}\right)$ novamente com os parâmetros significativos $\phi_{10}$ e $\phi_{21}$, os parâmetros estimados podem ser encontrados na Tabela 5.7 e os gráficos de STFAC e STFACP residuais nas Figuras 5.8 e 5.9.

\begin{tabular}{cccc}
\hline Parâmetro & Estimativa & Erro Padrão & P Valor \\
\hline$\phi_{10}$ & 0,6219 & 0,0554 & $<2,2 \times 10^{-16}$ \\
$\phi_{21}$ & 0,2905 & 0,0623 & $4,667 \times 10^{-6}$ \\
\hline
\end{tabular}

Tabela 5.7: Os parâmetros estimados do modelo $\operatorname{STAR}\left(2_{1}\right)$ com a matriz de pesos espaciais $e^{-d}$ 


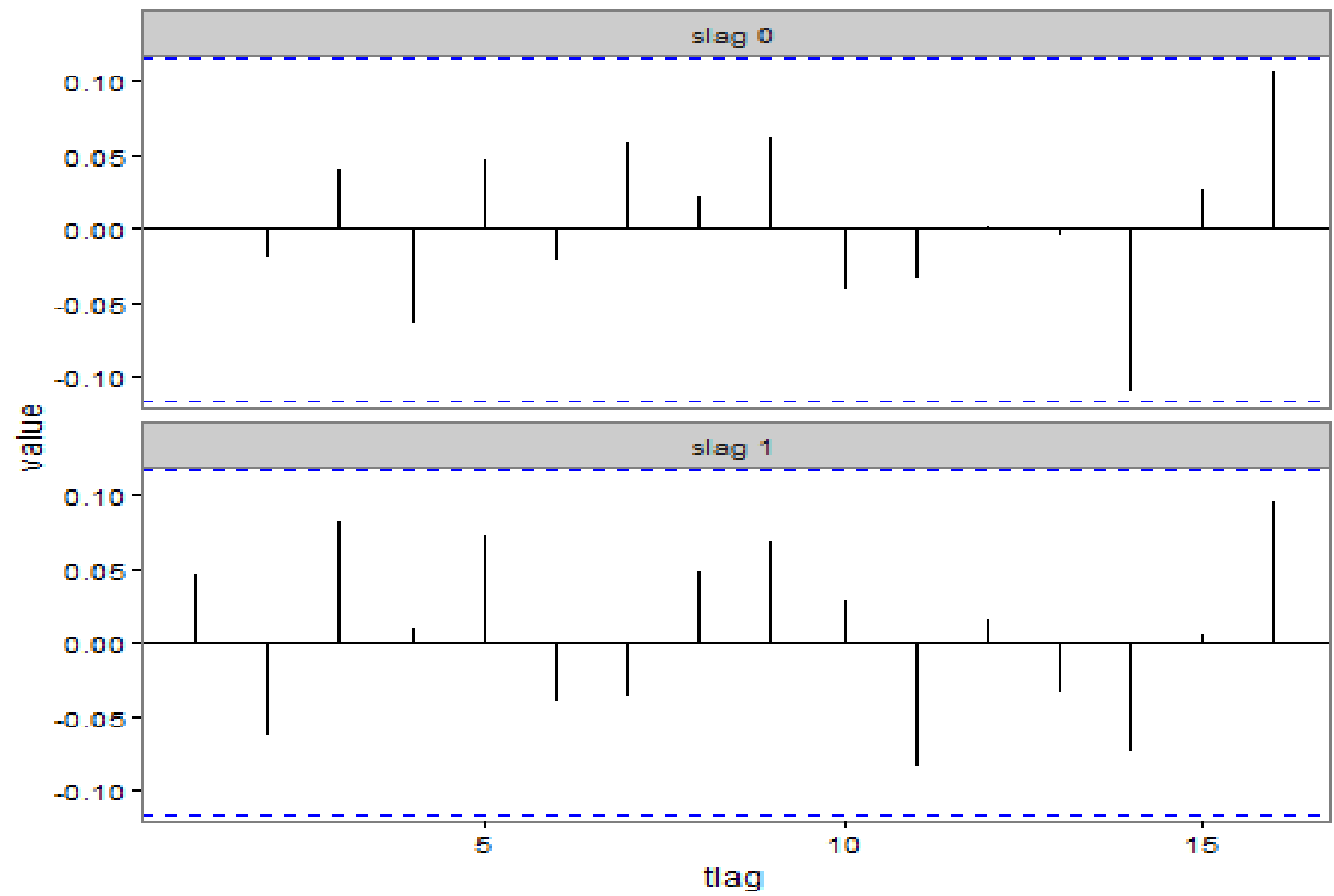

Figura 5.8: STFAC dos resíduos do modelo final $\operatorname{STAR}\left(2_{1}\right)$ utilizando a matriz de pesos espaciais $e^{-d}$

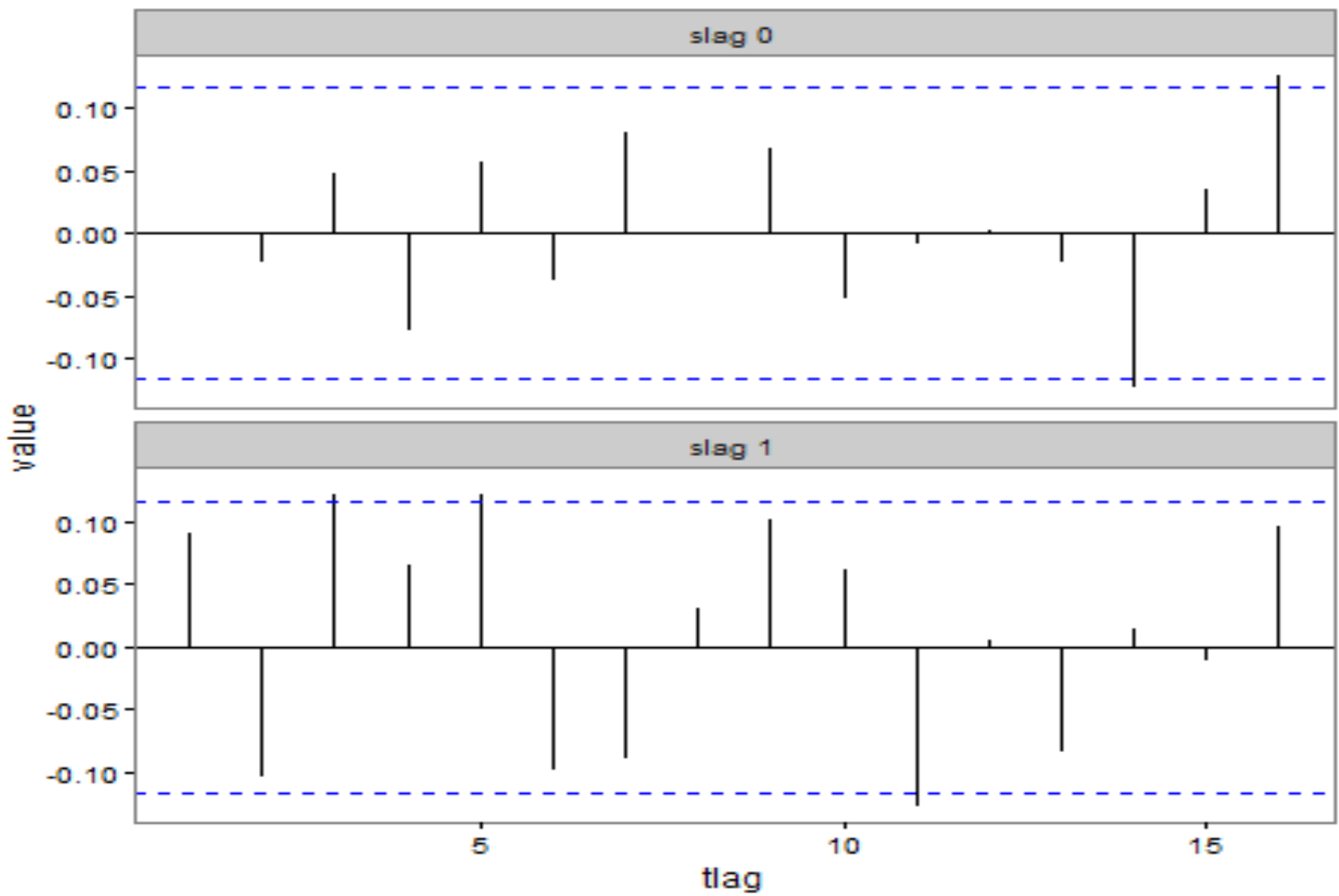

Figura 5.9: STFACP dos resíduos do modelo final $\operatorname{STAR}\left(2_{1}\right)$ utilizando a matriz de pesos espaciais $e^{-d}$ 
Adotamos também o peso espacial $e^{-2 d}$, e temos o melhor modelo $\operatorname{STAR}\left(2_{1}\right)$, os parâmetros significativos estimados estão na Tabela 5.8 e os seus respectivos gráficos de STFAC e STFACP residuais se encontram nas Figuras 5.10 e 5.11.

\begin{tabular}{cccc}
\hline Parâmetro & Estimativa & Erro Padrão & P Valor \\
\hline$\phi_{10}$ & 0,6217 & 0,0554 & $<2,2 \times 10^{-16}$ \\
$\phi_{21}$ & 0,2909 & 0,0623 & $4,659 \times 10^{-6}$ \\
\hline
\end{tabular}

Tabela 5.8: Os parâmetros estimados do modelo STAR $\left(2_{1}\right)$ com a matriz de pesos espaciais $e^{-2 d}$

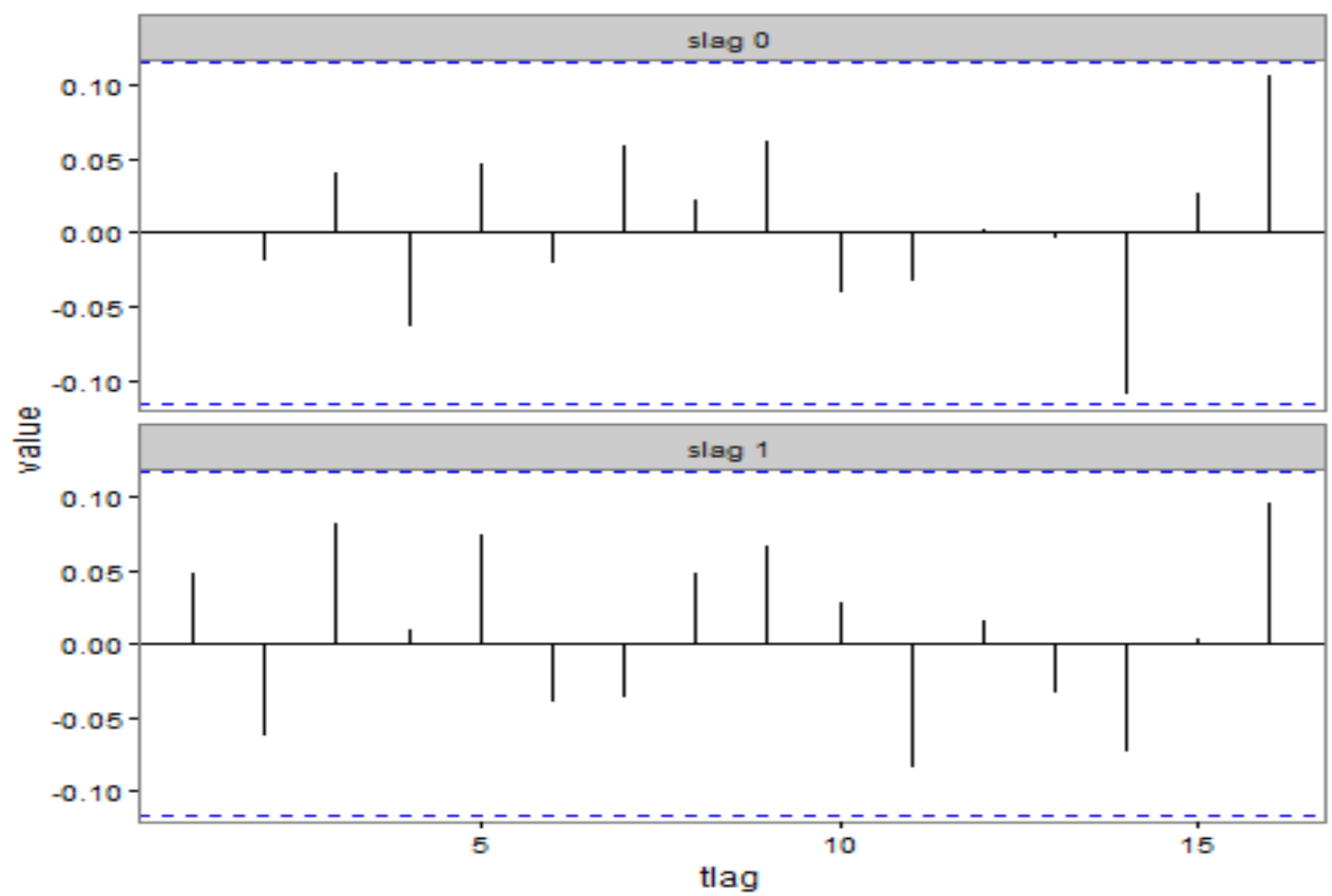

Figura 5.10: STFAC dos resíduos do modelo final $\operatorname{STAR}\left(2_{1}\right)$ utilizando a matriz de pesos espaciais $e^{-2 d}$ 


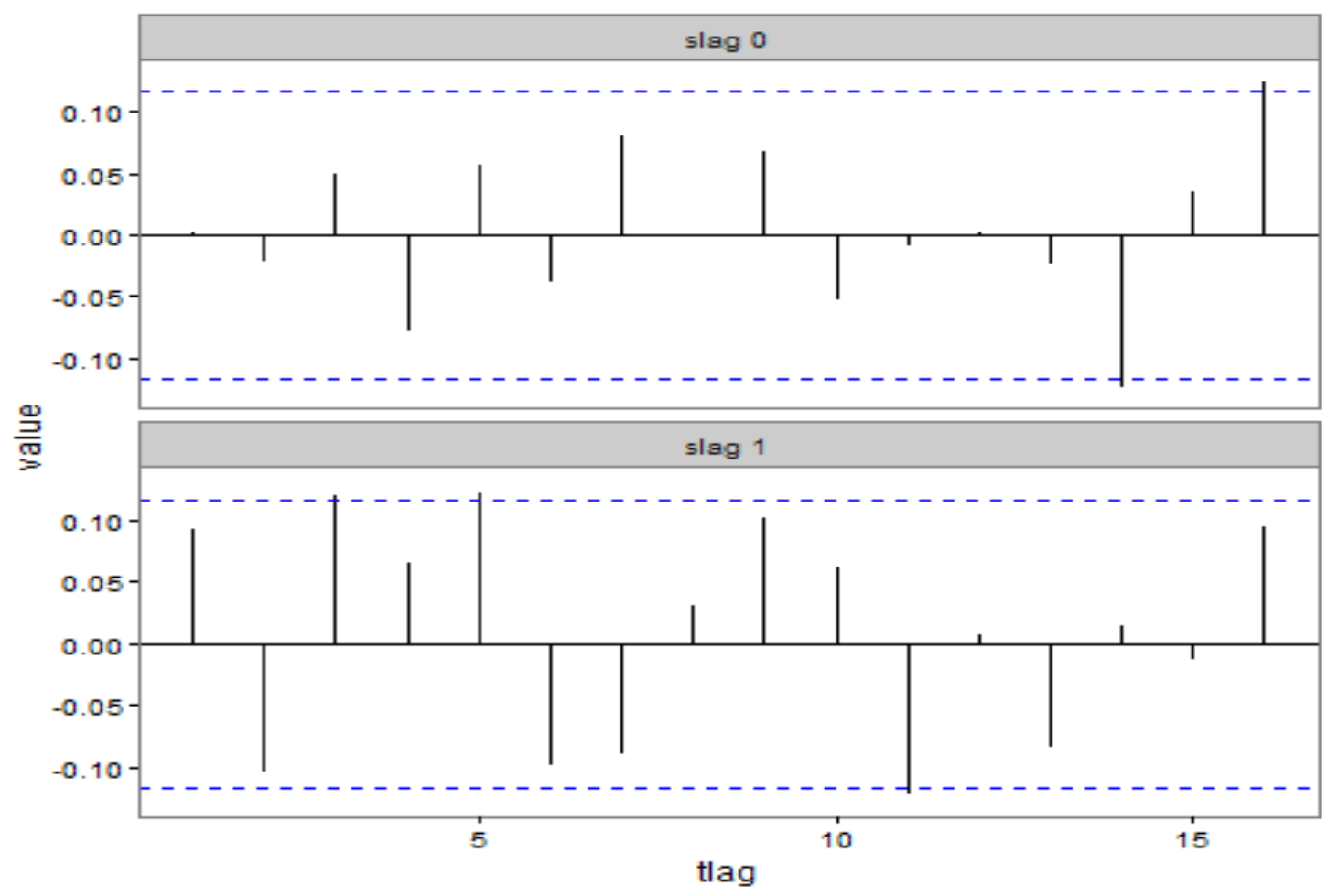

Figura 5.11: STFACP dos resíduos do modelo final STAR $\left(2_{1}\right)$ utilizando a matriz de pesos espaciais $e^{-2 d}$

Comparamos a qualidade de ajuste do modelo $\operatorname{STAR}\left(2_{1}\right)$ usando os pesos espaciais exponenciais para cada cidade, e pela Tabela 5.9 observamos que os EQM são extremamente próximos comprovando os resultados da simulação de que as estruturas de vizinhanças do tipo exponencial possuem uma estabilidade em relação à medida de ajuste dos modelos STARMA; e obtivemos novamente a semelhança entre a matriz espacial $e^{-0,5 d} \mathrm{e}$ a $e^{-d}$ por terem as qualidades de ajuste de modelo EQM quase iguais.

\begin{tabular}{cccc}
\hline & \multicolumn{3}{c}{ EQM } \\
\cline { 2 - 4 } Cidade & $e^{-0,5 d}$ & $e^{-d}$ & $e^{-2 d}$ \\
\hline Santo André & 0,28670 & 0,28669 & 0,28666 \\
São Bernardo do Campo & 0,13573 & 0,13578 & 0,13590 \\
São Caetano do Sul & 0,10998 & 0,10996 & 0,10992 \\
São Paulo & 0,13914 & 0,13917 & 0,13923 \\
Guarulhos & 0,65317 & 0,65312 & 0,65302 \\
Osasco & 0,35228 & 0,35226 & 0,35221 \\
\hline Geral & 0,27950 & 0,27950 & 0,27949 \\
\hline
\end{tabular}

Tabela 5.9: A qualidade de ajuste do modelo $\operatorname{STAR}\left(2_{1}\right)$ para cada cidade do estudo utilizando a matriz espacial $e^{-0,5 d}, e^{-d}$ e $e^{-2 d}$ 


\section{Capítulo 6}

\section{Conclusões e Estudos Futuros}

Neste trabalho, apresentamos a classe de modelos STARMA (Autorregressivo e Média Móvel Espaço-temporal), que é bastante utilizado para descrever dados de séries temporais espacialmente localizados, caracterizado pela dependência linear defasada tanto no espaço quanto no tempo. O modelo STARMA também pode ser uma ferramenta útil nos casos em que o pesquisador enfrenta conjuntos de dados de grande dimensão espacial e temporal, neste caso, é exigido apena um modelo, ao contrário do modelo ARIMA que tem que ser ajustado para cada série de estudo. Para construir um modelo STARMA, o cálculo da matriz de pesos espaciais $\boldsymbol{W}^{l}$ é fundamental. Existem diversas maneiras para calcular a $\boldsymbol{W}^{l}$, porém há poucos estudos que investigam se há melhor forma da matriz espacial no contexto de melhor ajuste de modelos STARMA.

No Capítulo 4, foi feito um estudo de simulação para comparar várias maneiras de cálculo da matriz de pesos espaciais. Os modelos não separáveis de covariância espaçotemporal Gneiting com diferentes combinações de parâmetros foram utilizados para simular as séries de 5 e 10 locais. Para cada modelo simulado, geramos 100 amostras de tamanho 100, e para cada amostra, ajustamos as séries pelos modelos $\operatorname{STAR}\left(1_{1}\right)$ especificando diferentes matrizes espaciais $\boldsymbol{W}^{l}$, que são os inversos da distância geodésica, $d^{-0,5}$, $d^{-1}, d^{-2}$, exponenciais da distância geodésica, $e^{-0,5 d}, e^{-d}, e^{-2 d}$ e a contiguidade espacial de ordem 1. As matrizes espaciais com pesos exponenciais apresentaram os melhores desempenhos de ajuste dos modelos STAR; e mostraram uma estabilidade em relação à medida de ajuste, ao contrário das matrizes $d^{-a}$ que têm uma variabilidade de EQM maior. A matriz de contiguidade mostrou o maior EQM nas simulações, lembrando que a matriz de contiguidade é definida de acordo com a habilidade do pesquisador de forma subjetiva.

No Capítulo 5, estudamos os comportamentos das séries do índice de preço imobiliário de venda de apartamentos com 2 domitórios das seis cidades do metropolitano de São Paulo. Percebemos que todas as séries possuem uma tendência crescente. Uma diferença foi adotada para as séries do índice, e foram ajustados vários modelos STARMA com a matriz de pesos espaciais $e^{-0,5 d}$. O modelo $\operatorname{STAR}\left(2_{1}\right)$ foi escolhido como o melhor modelo de ajuste por ter o menor EQM e os resíduos do modelo não mais correlacionados 
depois da modelagem. Foi ajustado para cada cidade um modelo ARMA univariado, e para cada cidade foi comparado o EQM calculado pelo modelo univariado ARMA e o EQM pelo STAR. Observamos que o modelo STAR ajusta melhor do que modelos univariados ARMA por ter mais cidades com EQM menores. Além disso, modelos STARMA custam um tempo menor de ajuste do que modelos univariados que exigem aplicação em cada série.

Estudos de interesse para o futuro:

1) Analisar dados espaço-temporais utilizando o modelo STARMA Generalizado caso a matriz de variância e covariância dos erros não seja homogênia, ou seja, $\Sigma_{\boldsymbol{\epsilon}_{t}} \neq$ $\sigma^{2} \boldsymbol{I}_{N T \times N T}$.

2) Pesquisar e estudar a classe de modelos STARMA para séries não estacionárias. Por exemplo ARFIMA espaço-temporal do Monroy (2013) e Cheng et al. $(2008)$.

3) Usar mais modelos de covariância espaço-temporal além do Gneiting para a simulação de comparação da matriz de pesos espaciais.

4) Seria interessante no futuro estudar outros modelos espaço-temporal, como modelo dinâmico espacial, o modelo hierárquico espaço-temporal etc; e compará-los com a classe de modelos STARMA. Os livros que podem ser consultados: Ozaki (2005), Kamarianakis et al. (2003), Elhorst (2001), Šaltyte (2005), Valentini et al. (2013), Carlin et al. (2004), Svetchnikova et al. (2008).

5) Pesquisar a estimação não paramétrica da classe de modelos STARMA. Por exemplo Anselin (2013). 


\section{Referências Bibliográficas}

Anselin(2013) Luc. Anselin. Spatial econometrics: methods and models, volume 4. Springer Science \& Business Media. Citado na pág. 1, 66

Bera e Jarque(1981) Anil K. Bera e Carlos M. Jarque. Efficient tests for normality, homoscedasticity and serial independence of regression residuals: Monte carlo evidence. Economics Letters, 7(4):313-318. Citado na pág. 17

Bivand et al.(2008) RS. Bivand, EJ. Pebesma e V. Gómez-Rubio. Applied Spatial Analysis with $R, 2008$. Citado na pág. 3

Biz(2014) Guilherme Biz. Simulações de pesos espaciais para o modelo STARMA e aplicações. Tese de Doutorado, Universidade de São Paulo Escola Superior de Agricultura Luiz de Queiroz, Piracicaba. Citado na pág. 21, 23, 25, 26, 29, 30

Box e Jenkins(1970) George E. P. Box e Gwilym M. Jenkins. Time Series Analysis, Forecasting and Control. San Francisco: Holden Day. Citado na pág. 4, 5, 8, 9, 19

Box et al.(1994) George EP. Box, Gwilym M. Jenkins e Gregory C. Reinsel. Time Analysis, Forecasting and Control, 1994. Citado na pág. 12, 13, 15

Brockwell e Davis(2006) Peter J. Brockwell e Richard A. Davis. Introduction to time series and forecasting. New York: Springer Science \& Business Media. Citado na pág. 8, 9, 13, 17

Carlin et al.(2004) Bradley P. Carlin, BP. Banerjee e Alan E. Gelfand. Hierarchical Modeling and Analysis for Spatial Data, 2004. Citado na pág. 66

Chatfield(2016) Chris. Chatfield. The analysis of time series: an introduction. New York: CRC press. Citado na pág. 9

Cheng et al.(2008) T. Cheng, JQ. Wang, X. Li e W. Zhang. A hybrid approach to model nonstationary space-time series. The International Archives of the Photogrammetry, Remote Sensing and Spatial Information Sciences, 37(B2):195-202. Citado na pág. 66 
Cipra e Motykova(1987) Tomáš. Cipra e I. Motykova. Study on kalman filter in time series analysis. Commentationes Mathematicae Universitatis Carolinae, 28(3):549-563. Citado na pág. 31

Clements et al.(2014) Nicolle. Clements, Sanat. Sarkar e William. Wei. Multiplicative spatio-temporal models for remotely sensed normalized difference vegetation index data. Journal of International Energy Policy (JIEP), 3(1):1-14. Citado na pág. 2

Cliff e Ord(1975) AD. Cliff e John K. Ord. Space-time modelling with an application to regional forecasting. Transactions of the Institute of British Geographers, páginas 119-128. Citado na pág. 19

Cressie e Huang(1999) Noel. Cressie e Hsin-Cheng. Huang. Classes of nonseparable, spatio-temporal stationary covariance functions. Journal of the American Statistical Association, 94(448):1330-1339. Citado na pág. 39, 40

Ehlers(2007) Ricardo S. Ehlers. Análise de séries temporais. Universidade Federal do Paraná. Citado na pág. 9, 13

Elhorst(2001) J Paul. Elhorst. Dynamic models in space and time. Geographical Analysis, 33(2):119-140. Citado na pág. 66

Fuller(2009) Wayne A. Fuller. Introduction to statistical time series, volume 428. New York: John Wiley and Sons. Citado na pág. 15

Gelfand et al.(2010) Alan E. Gelfand, Peter J. Diggle, Montserrat. Fuentes e Peter. Guttorp. Handbook of spatial statistics. CRC press. Citado na pág. 38

Getis(2009) Arthur. Getis. Spatial weights matrices. Geographical Analysis, 41(4):404410. Citado na pág. 21

Giacomini e Granger(2004) Raffaella. Giacomini e Clive WJ. Granger. Aggregation of space-time processes. Journal of econometrics, 118(1):7-26. Citado na pág. 1]

Glasbey e Allcroft(2008) CA. Glasbey e DJ. Allcroft. A spatiotemporal auto-regressive moving average model for solar radiation. Journal of the Royal Statistical Society: Series C (Applied Statistics), 57(3):343-355. Citado na pág. 2

Gneiting(2002) Tilmann. Gneiting. Nonseparable, stationary covariance functions for space-time data. Journal of the American Statistical Association, 97(458):590-600. Citado na pág. 40

Kamarianakis e Prastacos(2005) Yiannis. Kamarianakis e Poulicos. Prastacos. Spacetime modeling of traffic flow. Computers and Geosciences, 31(2):119-133. Citado na pág. 2 
Kamarianakis et al.(2003) Yiannis. Kamarianakis, Poulicos. Prastacos et al. Spatial time series modeling: A review of the proposed methodologies. The Regional Economics Applications Laboratory. Citado na pág. 66

Lamigueiro(2014) Oscar Perpinan. Lamigueiro. Displaying time series, spatial, and space-time data with $R$. New York: CRC Press. Citado na pág. 3

Ljung e Box(1978) Greta M. Ljung e George EP. Box. On a measure of lack of fit in time series models. Biometrika, 65(2):297-303. Citado na pág. 16

Martin e Oeppen(1975) Russell L. Martin e JE. Oeppen. The identification of regional forecasting models using space: time correlation functions. Transactions of the Institute of British Geographers, páginas 95-118. Citado na pág. 19, 26

Martins(2013) Natália da Silva Martins. Modelos autoregressivos e de médias móveis espaçoo-temporais (STARMA) aplicados a dados de temperatura. Tese de Doutorado, Universidade de São Paulo Escola Superior de Agricultura "Luiz de Queiroz", Piracicaba. Citado na pág. 2, 21, 24, 27

Monroy(2013) Natály A Jiménez. Monroy. Modelo ARFIMA Espaço-temporal em Estudos de Poluição do Ar. Tese de Doutorado, Universidade Federal do Espírito Santo, Vitória. Citado na pág. 66

Montgomery et al.(1990) Douglas C. Montgomery, Lynwood A. Johnson e John S. Gardiner. Forecasting and time series analysis. McGraw-Hill Companies. Citado na pág. 17

Morettin e Toloi(2006) Pedro A. Morettin e Clélia Toloi. Análise de Séries Temporais. São Paulo: Blucher. Citado na pág. 4, 6, 8, 9, 11, 13, 15, 17

Morettin(2008) Pedro Alberto. Morettin. Econometria financeira: um curso em séries temporais financeiras. São Paulo: Edgard Blücher. Citado na pág. 19, 55

Ozaki(2005) Vitor Augusto Ozaki. Métodos atuariais aplicados à determinação da taxa de prêmio de contratos de seguro agrícola: um estudo de caso. Tese de Doutorado, Universidade de São Paulo. Citado na pág. 66

Pace et al.(2000) R. Kelley. Pace, Ronaldo. Barry, Otis W. Gilley e C.F. Sirmans. A method for spatial-temporal forecasting with an application to real estate prices. International Journal of Forecasting, 16(2):229-246. Citado na pág. 1

Pfeifer e Deutsch(1980b) Phillip E. Pfeifer e Stuart Jay. Deutsch. Identification and interpretation of first order space-time arma models. Technometrics, 22(3):397-408. Citado na pág. 27 
Pfeifer e Deutsch(1980c) Phillip E. Pfeifer e Stuart Jay. Deutsch. A starima modelbuilding procedure with application to description and regional forecasting. Transactions of the Institute of British Geographers, páginas 330-349. Citado na pág. 24

Pfeifer e Deutsch(1980a) Phillip E. Pfeifer e Stuart Jay. Deutsch. A three-stage iterative procedure for space-time. Technometrics, 22(1):35-47. Citado na pág. 1, 19, 20, 21, 24, 25, 26, 28, 29, 30, 38,

Pfeifer e Deutsch(1981a) Phillip E. Pfeifer e Stuart Jay. Deutsch. Variance of the sample space-time autocorrelation function. Journal of the Royal Statistical Society. Series B (Methodological), páginas 28-33. Citado na pág. 36

Pfeifer e Deutsch(1981c) Phillip E. Pfeifer e Stuart Jay. Deutsch. Seasonal space-time arima modeling. Geographical analysis, 13(2):117-133. Citado na pág. 24

Rao e Antunes(2004) T Subba. Rao e Ana Monica Costa. Antunes. Spatio-temporal modelling of temperature time series: a comparative study. Em Time series analysis and applications to geophysical systems, páginas 123-150. Springer. Citado na pág. 1, 21. 30, 38,53

Šaltyte(2005) Laura. Šaltyte. Modified STARIMA model for space-time data, 2005. Citado na pág. 66

Sassi(2016) Gilberto Pereira. Sassi. Estimação de Modelos Geoestatísticos com Dados Funcionais usando Ondaletas. Tese de Doutorado, Universidade de São Paulo. Citado na pág. 1, 22

Shapiro e Wilk(1965) Samuel Sanford. Shapiro e Martin B. Wilk. An analysis of variance test for normality (complete samples). Biometrika, 52(3/4):591-611. Citado na pág. 17

Sherman(2011) Michael Sherman. Spatial statistics and spatio-temporal data: covariance functions and directional properties. John Wiley \& Sons. Citado na pág. 38, 39, 40, 41

Shoesmith(2013) Gary L. Shoesmith. Space-time autoregressive models and forecasting national, regional and state crime rates. International Journal of Forecasting, 29(1): 191-201. Citado na pág. 2

$\operatorname{Smith}(2013)$ Tony E. Smith. Spatial weight matrices. http://www.seas.upenn.edu/ ese502/lab-content/extra_materials, 2013. Accessed: 2016-11-01. Citado na pág. 21

Stein(2005) Michael L. Stein. Space-time covariance functions. Journal of the American Statistical Association, 100(469):310-321. Citado na pág. 40 
Svetchnikova et al.(2008) D. Svetchnikova, AN. Rambaldi e R. Strachan. A comparison of methods for spatial-temporal forecasting with an application to real estate prices. Em ESAM08 Markets and Models: Policy Frontiers in the AWH Phillips Tradition. Economic Society of Australia, NZ Association of Economists. Citado na pág. 66

Tsay(2005) Ruey S. Tsay. Analysis of financial time series, volume 543. John Wiley \& Sons. Citado na pág. 19

Tsay(2013) Ruey S Tsay. Multivariate time series analysis: with $R$ and financial applications. John Wiley \& Sons. Citado na pág. 19

Valentini et al.(2013) Pasquale. Valentini, Luigi. Ippoliti, Lara. Fontanella et al. Modeling us housing prices by spatial dynamic structural equation models. The Annals of Applied Statistics, 7(2):763-798. Citado na pág. 66

Wheelwright et al.(1998) Steven. Wheelwright, Spyros. Makridakis e Rob J. Hyndman. Forecasting: methods and applications. New York: John Wiley \& Sons. Citado na pág. 9

Zheng et al.(2014) Shanshan. Zheng, Qing. Wan e Mingyuan. Jia. Short-term forecasting of waterlogging at urban storm-waterlogging monitoring sites based on starma model. Progress in geography, 33(7):949-957. Citado na pág. 2, 53

Zhou e Buongiorno(2006) Mo. Zhou e Joseph. Buongiorno. Space-time modeling of timber prices. Journal of Agricultural and Resource Economics, páginas 40-56. Citado na pág. 2, 53 